\title{
Embedding Stochastic Correlation into the Pricing of FX Quanto Options under Stochastic Volatility Models*
}

\author{
Tommaso Pellegrino \\ Senior Quantitative Analyst in the Market Risk Quant Team, Danske Bank, Copenhagen, Denmark \\ Email: tommaso.pellegrino.research@gmail.com
}

How to cite this paper: Pellegrino, T. (2019) Embedding Stochastic Correlation into the Pricing of FX Quanto Options under Stochastic Volatility Models. Journal of Mathematical Finance, 9, 455-493. https://doi.org/10.4236/jmf.2019.93025

Received: July 9, 2019

Accepted: August 19, 2019

Published: August 22, 2019

Copyright () 2019 by author(s) and Scientific Research Publishing Inc. This work is licensed under the Creative Commons Attribution International License (CC BY 4.0).

http://creativecommons.org/licenses/by/4.0/

\section{(c) (i) Open Access}

\begin{abstract}
The aim of the paper is to incorporate a stochastic correlation structure when pricing quanto options under the assumption that both the underlying asset and the foreign exchange (FX) rate follow a stochastic volatility model. This is reached not only assuming that the correlation between the underlying asset and its variance process is stochastic (and the same between the exchange rate and its variance process), but also assuming a stochastic correlation between the underlying asset and the exchange rate. Under different stochastic correlation processes specifications, by approximating non-affine terms, we derive a closed-form approximation for the characteristic function of the underlying asset. Numerical experiments and comparison with Monte Carlo simulations are discussed. The analytical tractability of the formulas allows for fast pricing and calibration purposes.
\end{abstract}

\section{Keywords}

Stochastic Correlation Processes, Stochastic Volatility Models, Characteristic Function, FX Derivatives Pricing, Quanto Options

\section{Introduction}

A quantity-adjusting option, also known as a quanto option, is a cash-settled cross currency derivative, where the underlying asset is denominated in a currency (the so-called foreign currency) which is different from the one in which the option is settled (the so-called domestic currency). Investors use quanto options when they believe a particular asset will perform well in a country but they fear that country's currency will not perform as well. Thus, they will buy an op-

*The views, opinions, positions, results or strategies expressed in this article are those of the author and do not represent the views, opinions, positions, results or strategies of, and should not be attributed to, Danske Bank A/S or any of its affiliates. 
tion written on the foreign asset while keeping the payout in their domestic currency. This makes the option free from the foreign exchange rate fluctuation risk.

As pointed out in [1], the correlation between the underlying process and the exchange rate process plays a very important role in pricing. However, assuming a constant correlation between the underlying asset and the exchange rate is not realistic and might lead to correlation risk. In this paper, we choose to model correlation as a stochastic process.

In particular, our model specification attempts at unifying the setup of [2] and [1]. Indeed, in the first work, the authors extend the Heston model [3], incorporating a stochastic correlation between the underlying asset and its variance process, while in the second one, the same authors assume a stochastic correlation between the underlying asset and the exchange rate but within a constant volatility framework for both the underlying asset and the exchange rate.

In this paper, we relax the assumption of constant volatility for the underlying asset and the exchange rate, and we will assume that both of them follow a stochastic volatility model where, not only the correlation between the underlying asset and its variance is stochastic (and the same assumption holds for the exchange rate and its variance), but also the one between the underlying asset and the exchange rate is stochastic.

Adopting different stochastic correlation processes specifications, such as the Ornstein-Uhlenbeck and the bounded Jacobi processes, the characteristic function for the underlying asset can be derived in closed-form, by approximating all the non-affine terms in the model.

The remainder of the paper is organized as follows. In Section 2, we introduce the stochastic evolution for the underlying asset and for the exchange rate under the stochastic volatility model assumption with stochastic correlation structure. In Section 3 we show how to embed a stochastic correlation structure into the pricing of FX quanto options. In Section 4, we investigate the approximations of non-affine terms in the model, and by using different stochastic correlation processes specification, we derive the corresponding characteristic functions for the underlying asset in closed-form. In Section 5, we discuss how to discretize the model dynamics in order to run Monte Carlo simulations and in Section 6 we test the accuracy of the proposed approximation by comparing it with the Monte Carlo method. Conclusions are reported in Section 7.

\section{A Joint Stochastic Volatility Model for the Underlying Price and the FX Rate with Stochastic Correlation Structure}

Let $S(t)$ be the price at time $t$ for a certain underlying asset denominated in a foreign currency and let $X(t)$ be the (spot) exchange rate at time $t$ between the foreign and the domestic currencies. In what follows, we will assume that, under the historical measure $\mathbb{P}$, the stochastic dynamics for $S(t)$ and $X(t)$ are given by the following system of stochastic differential equations (SDEs): 


$$
\left\{\begin{array}{l}
\frac{\mathrm{d} S(t)}{S(t)}=\mu_{\mathrm{S}} \mathrm{d} t+\sqrt{V(t)} \mathrm{d} W_{\mathrm{S}}(t)^{\mathbb{P}}, S(0)>0, \\
\mathrm{~d} V(t)=\kappa_{\mathrm{V}}\left(\mu_{\mathrm{V}}-V(t)\right) \mathrm{d} t+\sigma_{\mathrm{V}} \sqrt{V(t)} \mathrm{d} W_{\mathrm{V}}(t)^{\mathbb{P}}, V(0)>0, \\
\frac{\mathrm{d} X(t)}{X(t)}=\mu_{\mathrm{X}} \mathrm{d} t+\sqrt{U(t)} \mathrm{d} W_{\mathrm{X}}(t)^{\mathbb{P}}, X(0)>0, \\
\mathrm{~d} U(t)=\kappa_{\mathrm{U}}\left(\mu_{\mathrm{U}}-U(t)\right) \mathrm{d} t+\sigma_{\mathrm{U}} \sqrt{U(t)} \mathrm{d} W_{\mathrm{U}}(t)^{\mathbb{P}}, U(0)>0,
\end{array}\right.
$$

where $V(t)$ and $U(t)$ are the variance processes for $S(t)$ and $X(t)$ respectively, following the well-known CIR process, as introduced in [4]. In particular, the stochastic dynamics for the pairs $(S(t), V(t)),(X(t), U(t))$ is nothing less than the Heston dynamics, but instead of assuming a constant correlation coefficient as in [3], we impose a stochastic correlation structure, in the sense that

$$
\operatorname{Corr}\left(\mathrm{d} W_{\mathrm{S}}(t)^{\mathbb{P}}, \mathrm{d} W_{\mathrm{V}}(t)^{\mathbb{P}}\right)=\eta(t) \mathrm{d} t \text { and } \operatorname{Corr}\left(\mathrm{d} W_{\mathrm{X}}(t)^{\mathbb{P}}, \mathrm{d} W_{\mathrm{U}}(t)^{\mathbb{P}}\right)=\gamma(t) \mathrm{d} t,
$$

where $\eta(t)$ and $\gamma(t)$ are stochastic processes, whose dynamics is given by:

$$
\left\{\begin{array}{l}
\mathrm{d} \eta(t)=a(t, \eta(t)) \mathrm{d} t+b(t, \eta(t)) \mathrm{d} W_{\eta}(t)^{\mathbb{P}}, \eta(0) \in[-1,1], \\
\mathrm{d} \gamma(t)=c(t, \gamma(t)) \mathrm{d} t+d(t, \gamma(t)) \mathrm{d} W_{\gamma}(t)^{\mathbb{P}}, \gamma(0) \in[-1,1],
\end{array}\right.
$$

for opportune coefficients $a(t, \eta(t)), b(t, \eta(t)), c(t, \gamma(t)), d(t, \gamma(t))$, which will depend on the assumption for the stochastic correlation process and which will be defined in Section 4 .

Therefore, as previously done in [2], we have assumed that the underlying asset $S(t)$ (respectively the exchange rate $X(t)$ ) is stochastically correlated with its own variance process $V(t)$ (resp. $U(t)$ ), with correlation process given by $\eta(t)$ (resp. $\gamma(t))$.

Besides that, we will assume that the underlying asset $S(t)$ and the exchange rate $X(t)$ are stochastically correlated, with correlation process $\beta(t)$, whose stochastic dynamics is given by:

$$
\mathrm{d} \beta(t)=f(t, \beta(t)) \mathrm{d} t+g(t, \beta(t)) \mathrm{d} W_{\beta}(t)^{\mathbb{P}}, \beta(0) \in[-1,1],
$$

once again for opportune coefficients $f(t, \beta(t)), g(t, \beta(t))$ to be specified.

We note that assuming a stochastic correlation between the underlying asset $S$ and the exchange rate $X$ has been proposed in [1], but in their work the authors assume a constant volatility structure for $S$ and $X$. Therefore, our model specification attempts at unifying the setup of [2] and [1].

As far as the other correlations are concerned, we will assume that the only non-zero correlations are as follows:

$$
\begin{aligned}
& \operatorname{Corr}\left(W_{\mathrm{S}}(t)^{\mathbb{P}}, W_{\beta}(t)^{\mathbb{P}}\right)=\rho_{\mathrm{S} \beta} \mathrm{d} t, \operatorname{Corr}\left(W_{\mathrm{S}}(t)^{\mathbb{P}}, W_{\eta}(t)^{\mathbb{P}}\right)=\rho_{\mathrm{S} \eta} \mathrm{d} t, \\
& \operatorname{Corr}\left(W_{\mathrm{X}}(t)^{\mathbb{P}}, W_{\beta}(t)^{\mathbb{P}}\right)=\rho_{\mathrm{X} \beta} \mathrm{d} t, \operatorname{Corr}\left(W_{\mathrm{X}}(t)^{\mathbb{P}}, W_{\gamma}(t)^{\mathbb{P}}\right)=\rho_{\mathrm{X} \gamma} \mathrm{d} t,
\end{aligned}
$$

i.e. we assume that the three stochastic correlation processes $(\eta(t), \gamma(t), \beta(t))$ are not correlated among each other, and that they are not correlated with the 
variance processes $(V(t), U(t))$ either. Finally, the variance processes themselves are not correlated between each other, and that $V(t)$ (resp. $U(t))$ is not correlated with $X(t)$ (resp. $S(t)$ ).

In summary, our model specification under the historical measure $\mathbb{P}$ reads as:

$$
\left\{\begin{array}{l}
\frac{\mathrm{d} S(t)}{S(t)}=\mu_{\mathrm{S}} \mathrm{d} t+\sqrt{V(t)} \mathrm{d} W_{\mathrm{S}}(t)^{\mathbb{P}}, \\
\mathrm{d} V(t)=\kappa_{\mathrm{V}}\left(\mu_{\mathrm{V}}-V(t)\right) \mathrm{d} t+\sigma_{\mathrm{V}} \sqrt{V(t)} \mathrm{d} W_{\mathrm{V}}(t)^{\mathbb{P}}, \\
\mathrm{d} \eta(t)=a(t, \eta(t)) \mathrm{d} t+b(t, \eta(t)) \mathrm{d} W_{\eta}(t)^{\mathbb{P}}, \eta(0) \in[-1,1], \\
\frac{\mathrm{d} X(t)}{X(t)}=\mu_{\mathrm{X}} \mathrm{d} t+\sqrt{U(t)} \mathrm{d} W_{\mathrm{X}}(t)^{\mathbb{P}}, \\
\mathrm{d} U(t)=\kappa_{\mathrm{U}}\left(\mu_{\mathrm{U}}-U(t)\right) \mathrm{d} t+\sigma_{\mathrm{U}} \sqrt{U(t)} \mathrm{d} W_{\mathrm{U}}(t)^{\mathbb{P}}, \\
\mathrm{d} \gamma(t)=c(t, \gamma(t)) \mathrm{d} t+d(t, \gamma(t)) \mathrm{d} W_{\gamma}(t)^{\mathbb{P}}, \gamma(0) \in[-1,1], \\
\mathrm{d} \beta(t)=f(t, \beta(t)) \mathrm{d} t+g(t, \beta(t)) \mathrm{d} W_{\beta}(t)^{\mathbb{P}}, \beta(0) \in[-1,1],
\end{array}\right.
$$

with the following correlation structure for the seven-dimensional Brownian motion

$$
\begin{aligned}
& \left(W_{\mathrm{S}}(t)^{\mathbb{P}}, W_{\mathrm{V}}(t)^{\mathbb{P}}, W_{\eta}(t)^{\mathbb{P}}, W_{\mathrm{X}}(t)^{\mathbb{P}}, W_{\mathrm{U}}(t)^{\mathbb{P}}, W_{\gamma}(t)^{\mathbb{P}}, W_{\beta}(t)^{\mathbb{P}}\right)^{\mathrm{T}} \\
& R=\left(\rho_{i j}\right)_{i j}=\left[\begin{array}{ccccccc}
1 & \eta(t) & \rho_{\mathrm{S} \eta} & \beta(t) & 0 & 0 & \rho_{\mathrm{S} \beta} \\
\eta(t) & 1 & 0 & 0 & 0 & 0 & 0 \\
\rho_{\mathrm{S} \eta} & 0 & 1 & 0 & 0 & 0 & 0 \\
\beta(t) & 0 & 0 & 1 & \gamma(t) & \rho_{\mathrm{X} \gamma} & \rho_{\beta \mathrm{X}} \\
0 & 0 & 0 & \gamma(t) & 1 & 0 & 0 \\
0 & 0 & 0 & \rho_{\mathrm{X} \gamma} & 0 & 1 & 0 \\
\rho_{\mathrm{S} \beta} & 0 & 0 & \rho_{\beta \mathrm{X}} & 0 & 0 & 1
\end{array}\right],
\end{aligned}
$$

which satisfies the common requirements of being symmetric and positive semi-definite.

By the argument of change of measure and using the Girsanov theorem, the model in Equation (6) can be specified under the risk-neutral measure $\mathbb{Q}$ as:

$$
\left\{\begin{array}{l}
\frac{\mathrm{d} S(t)}{S(t)}=\left(r_{\mathrm{f}}-\beta(t) \sqrt{V(t)} \sqrt{U(t)}\right) \mathrm{d} t+\sqrt{V(t)} \mathrm{d} W_{\mathrm{S}}(t)^{\mathbb{Q}}, \\
\mathrm{d} V(t)=\left[\kappa_{\mathrm{V}}\left(\mu_{\mathrm{V}}-V(t)\right)-\lambda_{\mathrm{V}}(t)\right] \mathrm{d} t+\sigma_{\mathrm{V}} \sqrt{V(t)} \mathrm{d} W_{\mathrm{V}}(t)^{\mathbb{Q}}, \\
\mathrm{d} \eta(t)=\left(a(t, \eta(t))-\lambda_{\eta}(t)\right) \mathrm{d} t+b(t, \eta(t)) \mathrm{d} W_{\eta}(t)^{\mathbb{Q}}, \eta(0) \in[-1,1], \\
\frac{\mathrm{d} X(t)}{X(t)}=\left(r_{\mathrm{d}}-r_{\mathrm{f}}\right) \mathrm{d} t+\sqrt{U(t)} \mathrm{d} W_{\mathrm{X}}(t)^{\mathbb{Q}}, \\
\mathrm{d} U(t)=\left[\kappa_{\mathrm{U}}\left(\mu_{\mathrm{U}}-U(t)\right)-\lambda_{\mathrm{U}}(t)\right] \mathrm{d} t+\sigma_{\mathrm{U}} \sqrt{U(t)} \mathrm{d} W_{\mathrm{U}}(t)^{\mathbb{Q}}, \\
\mathrm{d} \gamma(t)=\left(c(t, \gamma(t))-\lambda_{\gamma}(t)\right) \mathrm{d} t+d(t, \gamma(t)) \mathrm{d} W_{\gamma}(t)^{\mathbb{Q}}, \gamma(0) \in[-1,1], \\
\mathrm{d} \beta(t)=\left(f(t, \beta(t))-\lambda_{\beta}(t)\right) \mathrm{d} t+g(t, \beta(t)) \mathrm{d} W_{\beta}(t)^{\mathbb{Q}}, \beta(0) \in[-1,1],
\end{array}\right.
$$

with the same correlation structure as the one above, and where $\left(\lambda_{\mathrm{V}}(t), \lambda_{\mathrm{U}}(t)\right)$ 
and $\left(\lambda_{\eta}(t), \lambda_{\gamma}(t), \lambda_{\beta}(t)\right)$ represent the price of volatility and correlation risk respectively.

In particular, in what follows, we will assume that the market price of volatility risk is linear in the variance process, namely $\lambda_{\mathrm{V}}(t)=\lambda_{\mathrm{V}} V(t)$ (resp. $\left.\lambda_{\mathrm{U}}(t)=\lambda_{\mathrm{U}} U(t)\right)$, and that the market price of correlation risk is constant, in the sense that $\lambda_{\eta}(t)=\lambda_{\eta}$ and the same for $\lambda_{\gamma}(t)$ and $\lambda_{\beta}(t)$ respectively.

The risk-neutral dynamics for the variance process $V$ reads as ${ }^{1}$

$$
\begin{aligned}
\mathrm{d} V(t) & =\left(\kappa_{\mathrm{V}}+\lambda_{\mathrm{V}}\right)\left(\frac{\mu_{\mathrm{V}}}{\kappa_{\mathrm{V}}+\lambda_{\mathrm{V}}}-V(t)\right) \mathrm{d} t+\sigma_{\mathrm{V}} \sqrt{V(t)} \mathrm{d} W_{\mathrm{V}}(t)^{\mathbb{Q}} \\
& =\tilde{\kappa}_{\mathrm{V}}\left(\tilde{\mu}_{\mathrm{V}}-V(t)\right) \mathrm{d} t+\sigma_{\mathrm{V}} \sqrt{V(t)} \mathrm{d} W_{\mathrm{V}}(t)^{\mathbb{Q}},
\end{aligned}
$$

where we have defined $\tilde{\kappa}_{\mathrm{V}}=\kappa_{\mathrm{V}}+\lambda_{\mathrm{V}}, \quad \tilde{\mu}_{\mathrm{V}}=\frac{\mu_{\mathrm{V}}}{\kappa_{\mathrm{V}}+\lambda_{\mathrm{V}}}$ respectively.

Furthermore, under the log-transform for the underlying asset $S$ and for the exchange rate $X$, i.e. $Y(t)=\ln (S(t))$, and $Z(t)=\ln (X(t))$, the risk-neutral dynamics for $S$ and $X$ in the model of Equation (7) can be re-written as:

$$
\left\{\begin{array}{l}
\mathrm{d} Y(t)=\left(r_{\mathrm{f}}-\frac{1}{2} V(t)-\beta(t) \sqrt{V(t)} \sqrt{U(t)}\right) \mathrm{d} t+\sqrt{V(t)} \mathrm{d} W_{\mathrm{S}}(t)^{\mathbb{Q}}, \\
\mathrm{d} Z(t)=\left(r_{\mathrm{d}}-r_{\mathrm{f}}-\frac{1}{2} U(t)\right) \mathrm{d} t+\sqrt{U(t)} \mathrm{d} W_{\mathrm{X}}(t)^{\mathbb{Q}},
\end{array}\right.
$$

with the same dynamics for the variance and correlation processes as the one above.

\section{FX Quanto Options Pricing under Stochastic Volatility and Correlation}

Let $P(t)=P(t, Y(t), V(t), \eta(t), Z(t), U(t), \gamma(t), \beta(t))$ be the value at time $t$ of any contract written on the underlying asset $S$, denominated in the foreign currency, but paid in the domestic one.

Applying the multi-dimensional Feynman-Kac formula gives the following partial differential equation (PDE) satisfied by $P$ :

$$
\begin{aligned}
& \frac{\partial P}{\partial t}+\left(r_{\mathrm{f}}-\frac{1}{2} V(t)-\beta(t) \sqrt{V(t)} \sqrt{U(t)}\right) \frac{\partial P}{\partial y}+\left(r_{\mathrm{d}}-r_{\mathrm{f}}-\frac{1}{2} U(t)\right) \frac{\partial P}{\partial z} \\
& +\tilde{a}(t, \eta(t)) \frac{\partial P}{\partial \eta}+\kappa_{\mathrm{V}}\left(\mu_{\mathrm{V}}-V(t)\right) \frac{\partial P}{\partial v}+\kappa_{\mathrm{U}}\left(\mu_{\mathrm{U}}-U(t)\right) \frac{\partial P}{\partial u} \\
& +\tilde{c}(t, \gamma(t)) \frac{\partial P}{\partial \gamma}+\tilde{f}(t, \beta(t)) \frac{\partial P}{\partial \beta}+\frac{1}{2} V(t) \frac{\partial^{2} P}{\partial y^{2}}+\frac{1}{2} U(t) \frac{\partial^{2} P}{\partial z^{2}} \\
& +\frac{1}{2} \sigma_{\mathrm{V}}^{2} V(t) \frac{\partial^{2} P}{\partial v^{2}}+\frac{1}{2} \sigma_{\mathrm{U}}^{2} U(t) \frac{\partial^{2} P}{\partial u^{2}}+\frac{1}{2} b^{2}(t, \eta(t)) \frac{\partial^{2} P}{\partial \eta^{2}} \\
& +\frac{1}{2} d^{2}(t, \gamma(t)) \frac{\partial^{2} P}{\partial \gamma^{2}}+\frac{1}{2} g^{2}(t, \beta(t)) \frac{\partial^{2} P}{\partial \beta^{2}}+\beta(t) \sqrt{V(t)} \sqrt{U(t)} \frac{\partial^{2} P}{\partial y \partial z}
\end{aligned}
$$

${ }^{1}$ A similar discussion holds for the variance process $U$ as well. 


$$
\begin{aligned}
& +\sigma_{\mathrm{v}} \eta(t) V(t) \frac{\partial^{2} P}{\partial y \partial v}+\sigma_{\mathrm{U}} \gamma(t) U(t) \frac{\partial^{2} P}{\partial z \partial u}+\rho_{\mathrm{S} \beta} \sqrt{V(t)} g(t, \beta(t)) \frac{\partial^{2} P}{\partial y \partial \beta} \\
& +\rho_{\mathrm{S} \eta} \sqrt{V(t)} b(t, \eta(t)) \frac{\partial^{2} P}{\partial y \partial \eta}+\rho_{\mathrm{X} \beta} \sqrt{U(t)} g(t, \beta(t)) \frac{\partial^{2} P}{\partial z \partial \beta} \\
& +\rho_{\mathrm{X} \gamma} \sqrt{U(t)} d(t, \gamma(t)) \frac{\partial^{2} P}{\partial z \partial \gamma}-r_{\mathrm{d}} P=0 .
\end{aligned}
$$

In case where $P$ represents the price of a quanto (call) option, then its payoff (i.e. the terminal condition of the above PDE) at maturity time $T$, reads as:

$$
P(T)=\max (S(T)-K, 0),
$$

where $K$ is the strike price of the option.

Risk-neutral arguments imply as well that

$$
P(t)=P(t, S(t), X(t), T, K)=\mathrm{e}^{-r_{\mathrm{d}}(T-t)} X(t) \mathbb{E}^{\mathbb{Q}}\left[(S(T)-K)_{+} \mid \mathcal{F}(t)\right],
$$

where $\mathcal{F}(t)$ represents the flow of information available up to time $t$.

As done in [3], we assume that a solution of (10) has the following form:

$$
P(t, S(t), X(t), T, K)=\mathrm{e}^{-r_{\mathrm{d}}(T-t)}\left(\mathbb{E}^{\mathbb{Q}}[S(T) \mid \mathcal{F}(t)] P_{1}-K P_{2}\right),
$$

where the first term is the present value of the underlying asset upon optimal exercise, and the second term is the present value of the strike price payment (in the above equation we have also assumed that $X(t) \equiv 1)$.

Being more explicit, the probabilities $P_{1}, P_{2}$ are defined as follows:

$$
P_{j}=P_{j}(Y(T) \geq \ln K \mid \mathcal{F}(t)), \text { for } j=1,2,
$$

and they are nothing less than the conditional probabilities that the option expires in-the-money. It is well known that these probabilities are not immediately available in closed-form, however, we know that not only $P_{j}$, but also their corresponding characteristic functions $\phi_{j}=\mathbb{E}\left[\mathrm{e}^{i u X(T)} \mid \mathcal{F}(t)\right]$ satisfy the same PDE (8), subject to the terminal condition

$$
\phi_{j}(T)=\mathrm{e}^{i u X(T)}, \text { for } j=1,2 .
$$

From the knowledge of the characteristic functions $\phi_{j}$, one can get the probabilities $P_{j}$ by applying the following Fourier inversion formula

$$
P_{j}=\frac{1}{2}+\frac{1}{\pi} \int_{0}^{+\infty} \operatorname{Re}\left[\frac{\mathrm{e}^{-i \omega \ln K} \phi_{j}}{i \omega}\right] \mathrm{d} \omega .
$$

If we look back at Equation (11), we can observe that the only remaining quantity to be discussed is $\mathbb{E}^{\mathbb{Q}}[S(T) \mid \mathcal{F}(t)]$, which takes the following form, under the model specification of Equation (7):

$$
\mathbb{E}^{\mathbb{Q}}[S(T) \mid \mathcal{F}(t)]=\mathrm{e}^{r_{\mathrm{f}}(T-t)+\ln S(t)} \mathbb{E}^{\mathbb{Q}}\left[\mathrm{e}^{-\int_{t}^{T} \beta(s) \sqrt{U(s)} \sqrt{V(s)} \mathrm{d} s} \mid \mathcal{F}(t)\right] .
$$

Since, in general, there is no closed-form solution for the above expectation, we propose to approximate the term $\mathbb{E}^{\mathbb{Q}}\left[\mathrm{e}^{-\int_{t}^{T} \beta(s) \sqrt{U(s)} \sqrt{V(s)} \mathrm{d} s} \mid \mathcal{F}(t)\right]$ as follows: 


$$
\begin{aligned}
& \mathbb{E}^{\mathbb{Q}}\left[\mathrm{e}^{-\int_{t}^{T} \beta(s) \sqrt{U(s)} \sqrt{V(s)} \mathrm{d} s} \mid \mathcal{F}(t)\right] \\
& \approx \mathbb{E}^{\mathbb{Q}}\left[\mathrm{e}^{-\sqrt{U(t)} \sqrt{V(t)}]_{t}^{T} \beta(s) \mathrm{d} s} \mid \mathcal{F}(t)\right] \approx \mathbb{E}^{\mathbb{Q}}\left[\mathrm{e}^{-\sqrt{U(0)} \sqrt{V(0)} \int_{t}^{T} \beta(s) \mathrm{d} s} \mid \mathcal{F}(t)\right],
\end{aligned}
$$

where the last term on the right hand side can be calculated by deriving the characteristic function $\phi_{\xi}$ of the integrated correlation process $\xi=\int_{t}^{T} \beta(s) \mathrm{d} s$ under the risk-neutral measure and then set as argument $i \omega \sqrt{U(0)} \sqrt{V(0)}$.

Therefore, we have that

$$
\mathbb{E}[S(T) \mid \mathcal{F}(t)] \approx S(t) \mathrm{e}^{r_{\mathrm{f}}(T-t)} \phi_{\xi}(i \omega \sqrt{U(0)} \sqrt{V(0)}),
$$

from which it follows that our proposed solution would read as:

$$
P(t) \approx \mathrm{e}^{-r_{\mathrm{d}}(T-t)}\left(S(t) \mathrm{e}^{r_{\mathrm{f}}(T-t)} \phi_{\xi}(i \omega \sqrt{U(0)} \sqrt{V(0)}) P_{1}-K P_{2}\right) .
$$

Obviously, the system of SDEs in Equation (7) is not in the affine form, as some of the coefficients in the corresponding PDE (8) are not linear in the state variables $(y, v, \eta, z, u, \gamma, \beta)$. Therefore, as done in [2] and in [1], we linearize the coefficients of the pricing PDE in order to generate a system of SDEs which is in affine form. As we leave the specification of the drift and volatility coefficients for the correlation processes in Section 4, we first consider the following terms:

$$
\sigma_{\mathrm{v}} \eta(t) V(t) \text { and } \sigma_{\mathrm{U}} \gamma(t) U(t),
$$

which can be approximated (see for example [2]) as

$$
\sigma_{\mathrm{v}} \eta(t) V(t) \approx \sigma_{\mathrm{v}} \eta(t) \mathbb{E}^{\mathbb{Q}}[V(t)], \sigma_{\mathrm{U}} \gamma(t) U(t) \approx \sigma_{\mathrm{U}} \gamma(t) \mathbb{E}^{\mathbb{Q}}[U(t)],
$$

and this is justified from the fact that we have assumed zero correlation between the correlation process $\eta(t)$ (resp. $\gamma(t))$ and the variance process $V(t)$ (resp. $U(t)$ ).

Next, we propose to approximate the term $\beta(t) \sqrt{U(t)} \sqrt{V(t)}$ as follows:

$$
\beta(t) \sqrt{U(t)} \sqrt{V(t)} \approx \beta(t) \mathbb{E}^{\mathbb{Q}}[U(t)] \mathbb{E}^{\mathbb{Q}}[V(t)],
$$

where we use the fact that the correlation process $\beta(t)$ is not correlated with any of the variance processes $V$ and $U$, and also that $V$ and $U$ are not correlated between each other.

As said, the affinity of the terms including the drift and volatility coefficients of the stochastic correlation processes $\eta(t), \gamma(t)$ and $\beta(t)$ is discussed in the next section, as it will depend on the chosen stochastic process for modelling the correlations.

\section{Stochastic Correlation Processes Specification}

In this section, we apply an Ornstein-Uhlenbeck (OU) process and a bounded Jacobi (BJ) process in order to model stochastic correlation. As done in [2], we will approximate the non-affine terms involving the drift and the volatility coefficients of the correlation processes in order to be able to derive a closed-form solution for the price of a quanto option under the model in Equation (7). 


\subsection{The Ornstein-Uhlenbeck Process}

If we assume that the processes $\eta(t), \gamma(t)$ and $\beta(t)$ follow an OU process, then their drift and volatility coefficients are given by:

$$
\begin{aligned}
& \tilde{a}(t, \eta(t))=\kappa_{\eta}\left(\mu_{\mathrm{h}}-\eta(t)\right)-\lambda_{\eta}, \tilde{c}(t, \gamma(t))=\kappa_{\gamma}\left(\mu_{\gamma}-\gamma(t)\right)-\lambda_{\gamma}, \\
& \tilde{f}(t, \beta(t))=\kappa_{\beta}\left(\mu_{\beta}-\beta(t)\right)-\lambda_{\beta}, b(t, \eta(t))=\sigma_{\eta}, \\
& d(t, \gamma(t))=\sigma_{\gamma}, g(t, \beta(t))=\sigma_{\beta} .
\end{aligned}
$$

In particular, the drift coefficient for $\eta$ can be re-written as follows:

$$
\tilde{a}(t, \eta(t))=\kappa_{\eta}\left(\tilde{\mu}_{\eta}-\eta(t)\right), \text { with } \tilde{\mu}_{\eta}=\mu_{\eta}-\frac{\lambda_{\eta}}{\kappa_{\eta}},
$$

and the same holds for the drift coefficients for $\gamma$ and $\beta^{2}$.

Since the calibration of the model in Equation (7) is done directly under the Q measure, the usage of $\tilde{\mu}$ instead of $\mu$, is not relevant for the derivation of the results.

As pointed out in [2], one of the main drawbacks of using an OU process for stochastic correlation is the fact that the process is not bounded over the interval $[-1,1]$, and this specially happens for a small value of the mean reversion rate and a large value of the volatility parameter. Nevertheless, because of its analytical tractability, we will derive the results also under the assumption that the stochastic correlations follow an OU process.

Under the $\mathrm{OU}$ assumption for the correlation processes, the remaining non-affine terms can be approximated as follows:

$$
\left\{\begin{array}{l}
\rho_{\mathrm{S} \beta} \sigma_{\beta} \sqrt{V(t)} \approx \rho_{\mathrm{S} \beta} \sigma_{\beta} \mathbb{E}^{\mathbb{Q}}[\sqrt{V(t)}], \rho_{\mathrm{S} \eta} \sigma_{\eta} \sqrt{V(t)} \approx \rho_{\mathrm{S} \eta} \sigma_{\eta} \mathbb{E}^{\mathbb{Q}}[\sqrt{V(t)}], \\
\rho_{\mathrm{X} \beta} \sigma_{\beta} \sqrt{U(t)} \approx \rho_{\mathrm{X} \beta} \sigma_{\beta} \mathbb{E}^{\mathbb{Q}}[\sqrt{U(t)}], \rho_{\mathrm{X} \gamma} \sigma_{\gamma} \sqrt{U(t)} \approx \rho_{\mathrm{X} \gamma} \sigma_{\gamma} \mathbb{E}^{\mathbb{Q}}[\sqrt{U(t)}],
\end{array}\right.
$$

where the above approximation has been proposed in [5].

In particular, the following expectations $\mathbb{E}^{\mathbb{Q}}[\sqrt{V(t)}], \mathbb{E}^{\mathbb{Q}}[\sqrt{U(t)}]$ involving the square root of the variance processes $V$ and $U$ can be approximated as stated in the next proposition.

Proposition 1 (3.1 in [2]) Let $v(t)$ a stochastic process, who dynamics is given by:

$$
\mathrm{d} v(t)=\kappa_{v}\left(\mu_{v}-v(t)\right) \mathrm{d} t+\sigma_{v} \sqrt{v(t)} \mathrm{d} W_{v}(t)^{\mathbb{Q}} .
$$

Then, $\mathbb{E}^{\mathbb{Q}}[\sqrt{v(t)}]$ can be approximated by.

$$
\mathbb{E}^{\mathbb{Q}}[\sqrt{v(t)}] \approx m_{v}+n_{v} \mathrm{e}^{-l_{v} t},
$$

where $m_{v}, n_{v}$ and $l_{v}$ are defined as:

${ }^{2} \mathrm{We}$, indeed, have that $\tilde{c}(t, \gamma(t))=\kappa_{\gamma}\left(\tilde{\mu}_{\gamma}-\gamma(t)\right)$, with $\tilde{\mu}_{\gamma}=\mu_{\gamma}-\frac{\lambda_{\gamma}}{\kappa_{\gamma}}$, and

$\tilde{f}(t, \beta(t))=\kappa_{\beta}\left(\tilde{\mu}_{\beta}-\beta(t)\right)$, with $\tilde{\mu}_{\beta}=\mu_{\beta}-\frac{\lambda_{\beta}}{\kappa_{\beta}}$. 


$$
m_{v}=\sqrt{\mu_{v}-\frac{\sigma_{v}^{2}}{8 \kappa_{v}}}, n_{v}=\sqrt{v_{0}}-m_{v}, l_{v}=-\ln \left(\frac{\hat{d}_{v}-m_{v}}{n_{v}}\right),
$$

with

$$
\hat{d}_{v}=\sqrt{\left(v_{0} \mathrm{e}^{-\kappa_{v}}-\frac{\sigma_{v}^{2}\left(1-\mathrm{e}^{-\kappa_{v}}\right)}{4 \kappa_{v}}\right)+\mu_{v}\left(1-\mathrm{e}^{-\kappa_{v}}\right)+\frac{\sigma_{v}^{2} \mu_{v}\left(1-\mathrm{e}^{-\kappa_{v}}\right)^{2}}{8 \kappa_{v} \mu_{v}+8 \kappa_{v} \mathrm{e}^{-\kappa_{v}}\left(v_{0}-\mu_{v}\right)} .}
$$

Proof. See Appendix A.1.

We have therefore all the elements in order to find a solution for the pricing $\mathrm{PDE}$ (8), under the assumption that the correlation processes follow an $\mathrm{OU}$ process.

First, we observe that Equation (8) becomes

$$
\begin{aligned}
& \frac{\partial P}{\partial t}+\left(r_{\mathrm{f}}-\frac{1}{2} V(t)-\beta(t) \mathbb{E}^{\mathbb{Q}}[\sqrt{V(t)}] \mathbb{E}^{\mathbb{Q}}[\sqrt{U(t)}]\right) \frac{\partial P}{\partial y} \\
& +\left(r_{\mathrm{d}}-r_{\mathrm{f}}-\frac{1}{2} U(t)\right) \frac{\partial P}{\partial z}+\kappa_{\eta}\left(\mu_{\eta}-\eta(t)\right) \frac{\partial P}{\partial \eta}+\kappa_{\mathrm{V}}\left(\mu_{\mathrm{V}}-V(t)\right) \frac{\partial P}{\partial v} \\
& +\kappa_{\mathrm{U}}\left(\mu_{\mathrm{U}}-U(t)\right) \frac{\partial P}{\partial u}+\kappa_{\gamma}\left(\mu_{\gamma}-\gamma(t)\right) \frac{\partial P}{\partial \gamma}+\kappa_{\beta}\left(\mu_{\beta}-\beta(t)\right) \frac{\partial P}{\partial \beta} \\
& +\frac{1}{2} V(t) \frac{\partial^{2} P}{\partial y^{2}}+\frac{1}{2} U(t) \frac{\partial^{2} P}{\partial z^{2}}+\frac{1}{2} \sigma_{\mathrm{V}}^{2} V(t) \frac{\partial^{2} P}{\partial \nu^{2}}+\frac{1}{2} \sigma_{\mathrm{U}}^{2} U(t) \frac{\partial^{2} P}{\partial u^{2}} \\
& +\frac{1}{2} \sigma_{\eta}^{2} \frac{\partial^{2} P}{\partial \eta^{2}}+\frac{1}{2} \sigma_{\gamma}^{2} \frac{\partial^{2} P}{\partial \gamma^{2}}+\frac{1}{2} \sigma_{\beta}^{2} \frac{\partial^{2} P}{\partial \beta^{2}}+\beta(t) \mathbb{E}^{\mathbb{Q}}[\sqrt{V(t)}] \mathbb{E}^{\mathbb{Q}}[\sqrt{U(t)}] \frac{\partial^{2} P}{\partial y \partial z} \\
& +\sigma_{\mathrm{v}} \mathbb{E}^{\mathbb{Q}}[V(t)] \eta(t) \frac{\partial^{2} P}{\partial y \partial \partial}+\sigma_{\mathrm{U}} \mathbb{E}^{\mathbb{Q}}[U(t)] \gamma(t) \frac{\partial^{2} P}{\partial z \partial u} \\
& +\rho_{\mathrm{S} \beta} \sigma_{\beta} \mathbb{E}^{\mathbb{Q}}[\sqrt{V(t)}] \frac{\partial^{2} P}{\partial y \partial \beta}+\rho_{\mathrm{S} \eta} \sigma_{\eta} \mathbb{E}^{\mathbb{Q}}[\sqrt{V(t)}] \frac{\partial^{2} P}{\partial y \partial \eta} \\
& +\rho_{\mathrm{X} \beta} \sigma_{\beta} \mathbb{E}^{\mathbb{Q}}[\sqrt{U(t)}] \frac{\partial^{2} P}{\partial z \partial \beta}+\rho_{\mathrm{x} \gamma} \sigma_{\gamma} E^{\mathbb{Q}}[\sqrt{U(t)}] \frac{\partial^{2} P}{\partial z \partial \gamma}-r_{\mathrm{d}} P=0 .
\end{aligned}
$$

By substituting (18) into the pricing PDE (29), we obtain the following PDEs in $P_{1}$ and $P_{2}$ respectively:

$$
\begin{aligned}
& \frac{\partial P_{1}}{\partial t}+\left(r_{\mathrm{f}}+\frac{1}{2} V(t)-\beta(t) \mathbb{E}[\sqrt{V(t)}] \mathbb{E}[\sqrt{U(t)}]\right) \frac{\partial P_{1}}{\partial y}+\left(r_{\mathrm{d}}-r_{\mathrm{f}}-\frac{1}{2} U(t)\right. \\
& +\beta(t) \mathbb{E}[\sqrt{V(t)}] \mathbb{E}[\sqrt{U(t)}]) \frac{\partial P_{1}}{\partial z}+\left(\kappa_{\eta}\left(\mu_{\eta}-\eta(t)\right)+\rho_{\mathrm{S} \eta} \sigma_{\eta} \mathbb{E}[\sqrt{V(t)}]\right) \frac{\partial P_{1}}{\partial \eta} \\
& +\left(\kappa_{\mathrm{V}}\left(\mu_{\mathrm{V}}-V(t)\right)+\sigma_{\mathrm{V}} \mathbb{E}[V(t)] \eta(t)\right) \frac{\partial P_{1}}{\partial v}+\kappa_{\mathrm{U}}\left(\mu_{\mathrm{U}}-U(t)\right) \frac{\partial P_{1}}{\partial u} \\
& +\left(\kappa_{\beta}\left(\mu_{\beta}-\beta(t)\right)+\rho_{\mathrm{S} \beta} \sigma_{\beta} \mathbb{E}[\sqrt{V(t)}]\right) \frac{\partial P_{1}}{\partial \beta}+\kappa_{\gamma}\left(\mu_{\gamma}-\gamma(t)\right) \frac{\partial P_{1}}{\partial \gamma} \\
& +\frac{1}{2} V(t) \frac{\partial^{2} P_{1}}{\partial y^{2}}+\frac{1}{2} U(t) \frac{\partial^{2} P_{1}}{\partial z^{2}}+\frac{1}{2} \sigma_{\mathrm{V}}^{2} V(t) \frac{\partial^{2} P_{1}}{\partial v^{2}}+\frac{1}{2} \sigma_{\mathrm{U}}^{2} U(t) \frac{\partial^{2} P_{1}}{\partial u^{2}} \\
& +\frac{1}{2} \sigma_{\beta}^{2} \frac{\partial^{2} P_{1}}{\partial \beta^{2}}+\frac{1}{2} \sigma_{\eta}^{2} \frac{\partial^{2} P_{1}}{\partial \eta^{2}}+\frac{1}{2} \sigma_{\gamma}^{2} \frac{\partial^{2} P_{1}}{\partial \gamma^{2}}+\beta(t) \mathbb{E}[\sqrt{V(t)}] \mathbb{E}[\sqrt{U(t)}] \frac{\partial^{2} P_{1}}{\partial y \partial z}
\end{aligned}
$$




$$
\begin{aligned}
& +\sigma_{\mathrm{V}} \mathbb{E}[V(t)] \eta(t) \frac{\partial^{2} P_{1}}{\partial y \partial v}+\sigma_{\mathrm{U}} \mathbb{E}[U(t)] \gamma(t) \frac{\partial^{2} P_{1}}{\partial z \partial u} \\
& +\rho_{\mathrm{S} \beta} \sigma_{\beta} \mathbb{E}[\sqrt{V(t)}] \frac{\partial^{2} P_{1}}{\partial \beta \partial y}+\rho_{\mathrm{S} \eta} \sigma_{\eta} \mathbb{E}[\sqrt{V(t)}] \frac{\partial^{2} P_{1}}{\partial \eta \partial y} \\
& +\rho_{\mathrm{X} \beta} \sigma_{\beta} \mathbb{E}[\sqrt{U(t)}] \frac{\partial^{2} P_{1}}{\partial z \partial \beta}+\rho_{\mathrm{X} \gamma} \sigma_{\gamma} \mathbb{E}[\sqrt{U(t)}] \frac{\partial^{2} P_{1}}{\partial z \partial \gamma}=0,
\end{aligned}
$$

and

$$
\begin{aligned}
& \frac{\partial P_{2}}{\partial t}+\left(r_{\mathrm{f}}-\frac{1}{2} V(t)-\beta(t) \mathbb{E}[\sqrt{V(t)}] \mathbb{E}[\sqrt{U(t)}]\right) \frac{\partial P_{2}}{\partial y} \\
& +\left(r_{\mathrm{d}}-r_{\mathrm{f}}-\frac{1}{2} U(t)\right) \frac{\partial P_{2}}{\partial z}+\kappa_{\eta}\left(\mu_{\eta}-\eta(t)\right) \frac{\partial P_{2}}{\partial \eta}+\kappa_{\mathrm{V}}\left(\mu_{\mathrm{V}}-V(t)\right) \frac{\partial P_{2}}{\partial v} \\
& +\kappa_{\mathrm{U}}\left(\mu_{\mathrm{U}}-U(t)\right) \frac{\partial P_{2}}{\partial u}+\kappa_{\beta}\left(\mu_{\beta}-\beta(t)\right) \frac{\partial P_{2}}{\partial \beta}+\kappa_{\gamma}\left(\mu_{\gamma}-\gamma(t)\right) \frac{\partial P_{2}}{\partial \gamma} \\
& +\frac{1}{2} V(t) \frac{\partial^{2} P_{2}}{\partial y^{2}}+\frac{1}{2} U(t) \frac{\partial^{2} P_{2}}{\partial z^{2}}+\frac{1}{2} \sigma_{\mathrm{V}}^{2} V(t) \frac{\partial^{2} P_{2}}{\partial v^{2}}+\frac{1}{2} \sigma_{\mathrm{U}}^{2} U(t) \frac{\partial^{2} P_{2}}{\partial u^{2}} \\
& +\frac{1}{2} \sigma_{\beta}^{2} \frac{\partial^{2} P_{2}}{\partial \beta^{2}}+\frac{1}{2} \sigma_{\eta}^{2} \frac{\partial^{2} P_{2}}{\partial \eta^{2}}+\frac{1}{2} \sigma_{\gamma}^{2} \frac{\partial^{2} P_{2}}{\partial \gamma^{2}}+\beta(t) \mathbb{E}[\sqrt{V(t)}] \mathbb{E}[\sqrt{U(t)}] \frac{\partial^{2} P_{2}}{\partial y \partial z} \\
& +\sigma_{\mathrm{V}} \mathbb{E}[V(t)] \eta(t) \frac{\partial^{2} P_{2}}{\partial y \partial v}+\sigma_{\mathrm{U}} \mathbb{E}_{\mathbb{U}}[U(t)] \gamma(t) \frac{\partial^{2} P_{2}}{\partial z \partial u} \\
& +\rho_{\mathrm{S} \beta} \sigma_{\beta} \mathbb{E}[\sqrt{V(t)}] \frac{\partial^{2} P_{2}}{\partial \beta \partial y}+\rho_{\mathrm{S} \eta} \sigma_{\eta} \mathbb{E}[\sqrt{V(t)}] \frac{\partial^{2} P_{2}}{\partial \eta \partial y} \\
& +\rho_{\mathrm{X} \beta} \sigma_{\beta} \mathbb{E}[\sqrt{U(t)}] \frac{\partial^{2} P_{2}}{\partial z \partial \beta}+\rho_{\mathrm{X} \gamma} \sigma_{\gamma} \mathbb{E}[\sqrt{U(t)}] \frac{\partial^{2} P_{2}}{\partial z \partial \gamma}=0 .
\end{aligned}
$$

As pointed out in the previous section, we know that the corresponding characteristic functions $\phi=\phi_{j}(t, y, z, v, u, \beta, \eta, \gamma ; \omega)$ of $P_{j}, j=1,2$, must also satisfy the PDEs (30) and (31), respectively. Their solutions can be found in a closed-form as stated in the next lemmas.

Lemma 1. The characteristic function of $P_{1}$ in (18), under the assumption that the correlation processes $\beta(t), \eta(t)$ and $\gamma(t)$ follow an OU process, is given by:

$$
\begin{aligned}
\phi_{1}^{\mathrm{oU}}= & \exp \left(A_{1}(\tau, \omega)+B_{1}(\tau, \omega) v(t)+C_{1}(\tau, \omega) u(t)+D_{1}(\tau, \omega) z(t)\right. \\
& \left.+E_{1}(\tau, \omega) \beta(t)+F_{1}(\tau, \omega) \eta(t)+G_{1}(\tau, \omega) \gamma(t)+i \omega y(t)\right),
\end{aligned}
$$

where $C_{1}(\tau, \omega)=D_{1}(\tau, \omega)=G_{1}(\tau, \omega) \equiv 0$ and where

$$
\begin{gathered}
B_{1}(\tau, \omega)=\frac{\kappa_{\mathrm{V}}-\bar{B}_{1}}{\sigma_{\mathrm{V}}^{2}} \frac{1-\mathrm{e}^{-\bar{B}_{1} \tau}}{1-\bar{B}_{2} \mathrm{e}^{-\bar{B}_{1} \tau}}, \\
E_{1}(\tau, \omega)=-i \omega\left[\frac{m_{\mathrm{V}} m_{\mathrm{U}}}{\kappa_{\beta}}\left(1-\mathrm{e}^{-\kappa_{\beta} \tau}\right)+\frac{m_{\mathrm{V}} n_{\mathrm{U}}}{\kappa_{\beta}+l_{\mathrm{U}}} \mathrm{e}^{-l_{\mathrm{U}}(T-\tau)}\left(1-\mathrm{e}^{-\left(\kappa_{\beta}+l_{\mathrm{U}}\right) \tau}\right)\right. \\
+\frac{m_{\mathrm{U}} n_{\mathrm{V}}}{\kappa_{\beta}+l_{\mathrm{V}}} \mathrm{e}^{-l_{\mathrm{V}}(T-\tau)}\left(1-\mathrm{e}^{-\left(\kappa_{\beta}+l_{\mathrm{V}}\right) \tau}\right) \\
\left.+\frac{n_{\mathrm{U}} n_{\mathrm{V}}}{\kappa_{\beta}+l_{\mathrm{U}}+l_{\mathrm{V}}} \mathrm{e}^{-\left(l_{\mathrm{U}}+l_{\mathrm{V}}\right)(T-\tau)}\left(1-\mathrm{e}^{-\left(\kappa_{\beta}+l_{\mathrm{U}}+l_{\mathrm{V}}\right) \tau}\right)\right],
\end{gathered}
$$




$$
\begin{aligned}
F_{1}(\tau, \omega)= & \frac{\left(\mu_{\mathrm{V}}-v(0)\right) \hat{B}_{1}}{\kappa_{\eta}+\kappa_{\mathrm{V}}-\bar{B}_{3}} \mathrm{e}^{\left(\kappa_{\mathrm{V}}-\bar{B}_{3}\right) \tau-\kappa_{\mathrm{V}} T}+\frac{\left(v(0)-\mu_{\mathrm{V}}\right) \hat{B}_{1}}{\kappa_{\eta}+\kappa_{\mathrm{V}}} \mathrm{e}^{\kappa_{\mathrm{V}}(\tau-T)} \\
& +\frac{\mu_{\mathrm{V}} \hat{B}_{1}}{\kappa_{\eta}}-\frac{\mu_{\mathrm{V}} \hat{B}_{1}}{\kappa_{\eta}-\bar{B}_{3}} \mathrm{e}^{-\bar{B}_{3} \tau}+\hat{B}_{1} \hat{B}_{2} \mathrm{e}^{-\kappa_{\eta} \tau}, \\
A_{1}(\tau, \omega)= & r_{\mathrm{f}} i \omega \tau+\kappa_{\mathrm{V}} \mu_{\mathrm{V}} H_{1}(\tau, \omega)+\left(\kappa_{\eta} \mu_{\eta}+(i \omega+1) \rho_{\mathrm{S} \eta} \sigma_{\eta} m_{\mathrm{V}}\right) H_{2}(\tau, \omega) \\
& +(i \omega+1) \rho_{\mathrm{S} \eta} \sigma_{\eta} n_{\mathrm{V}} H_{3}(\tau, \omega) \\
& +\left(\kappa_{\beta} \mu_{\beta}+(i \omega+1) \rho_{\mathrm{S} \beta} \sigma_{\beta} m_{\mathrm{V}}\right) H_{4}(\tau, \omega) \\
& +(i \omega+1) \rho_{\mathrm{S} \beta} \sigma_{\beta} n_{\mathrm{V}} H_{5}(\tau, \omega)+\frac{1}{2} \sigma_{\beta}^{2} H_{6}(\tau, \omega)+\frac{1}{2} \sigma_{\eta}^{2} H_{7}(\tau, \omega) .
\end{aligned}
$$

The H functions in Equation (36) are defined as.

$$
\begin{aligned}
& H_{1}(\tau, \omega)=\int_{0}^{\tau} B_{1}(s, \omega) \mathrm{d} s, H_{2}(\tau, \omega)=\int_{0}^{\tau} F_{1}(s, \omega) \mathrm{d} s, \\
& H_{3}(\tau, \omega)=\int_{0}^{\tau} \mathrm{e}^{-l_{\mathrm{V}}(T-s)} F_{1}(s, \omega) \mathrm{d} s, H_{4}(\tau, \omega)=\int_{0}^{\tau} E_{1}(s, \omega) \mathrm{d} s, \\
& H_{5}(\tau, \omega)=\int_{0}^{\tau} \mathrm{e}^{-l_{\mathrm{V}}(T-s)} E_{1}(s, \omega) \mathrm{d} s, H_{6}(\tau, \omega)=\int_{0}^{\tau} E_{1}(s, \omega)^{2} \mathrm{~d} s, \\
& H_{7}(\tau, \omega)=\int_{0}^{\tau} F_{1}(s, \omega)^{2} \mathrm{~d} s,
\end{aligned}
$$

and the coefficients $\bar{B}_{1}, \bar{B}_{2}, \bar{B}_{3}, \hat{B}_{1}, \hat{B}_{2}$ are respectively given by

$$
\begin{gathered}
\bar{B}_{1}=\sqrt{\kappa_{\mathrm{V}}^{2}+\sigma_{\mathrm{V}}^{2} \omega(\omega-i)}, \\
\bar{B}_{2}=\frac{-\kappa_{\mathrm{V}}+\bar{B}_{1}}{\kappa_{\mathrm{V}}+\bar{B}_{1}}, \\
\bar{B}_{3}=-\ln \left(\frac{\mathrm{e}^{-\bar{B}_{1}}-\bar{B}_{2} \mathrm{e}^{-\bar{B}_{1}}}{\left.1-\bar{B}_{2} \mathrm{e}^{-\bar{B}_{1}}\right),}\right. \\
\hat{B}_{1}=\frac{i \omega+1}{\sigma_{\mathrm{V}}}\left(\kappa_{\mathrm{V}}-\bar{B}_{1}\right), \\
\hat{B}_{2}=-\frac{\mu_{\mathrm{V}}}{\kappa_{\mathrm{V}}}-\frac{v(0)-\mu_{\mathrm{V}}}{\kappa_{\eta}+\kappa_{\mathrm{V}}} \mathrm{e}^{-\kappa_{\mathrm{V}} T}-\frac{\mu_{\mathrm{V}}-v(0)}{\kappa_{\eta}+\kappa_{\mathrm{V}}-\bar{B}_{3}} \mathrm{e}^{-\kappa_{\mathrm{V}} T}+\frac{\mu_{\mathrm{V}}}{\kappa_{\eta}-\bar{B}_{3}} .
\end{gathered}
$$

with $m_{\mathrm{U}}, m_{\mathrm{V}}, n_{\mathrm{U}}, n_{\mathrm{V}}, l_{\mathrm{U}}, l_{\mathrm{V}}$ as in Proposition 1 .

Proof. See Appendix A.2.

Lemma 2. The characteristic function of $P_{2}$ in (18), under the assumption that the correlation processes $\beta(t), \eta(t)$ and $\gamma(t)$ follow an $O U$ process, is given by:

$$
\begin{aligned}
\phi_{2}^{\mathrm{oU}}= & \exp \left(A_{2}(\tau, \omega)+B_{2}(\tau, \omega) v(t)+C_{2}(\tau, \omega) u(t)+D_{2}(\tau, \omega) z(t)\right. \\
& \left.+E_{2}(\tau, \omega) \beta(t)+F_{2}(\tau, \omega) \eta(t)+G_{2}(\tau, \omega) \gamma(t)+i \omega y(t)\right),
\end{aligned}
$$

where $C_{2}(\tau, \omega)=D_{2}(\tau, \omega)=G_{2}(\tau, \omega) \equiv 0$ and where

$$
B_{2}(\tau, \omega)=\frac{\kappa_{\mathrm{V}}-\tilde{B}_{1}}{\sigma_{\mathrm{V}}^{2}} \frac{1-\mathrm{e}^{-\tilde{B}_{1} \tau}}{1-\tilde{B}_{2} \mathrm{e}^{-\tilde{B}_{1} \tau}},
$$




$$
\begin{aligned}
E_{2}(\tau, \omega)= & i \omega\left[\frac{m_{\mathrm{V}} m_{\mathrm{U}}}{\kappa_{\beta}}\left(1-\mathrm{e}^{-\kappa_{\beta} \tau}\right)+\frac{m_{\mathrm{V}} n_{\mathrm{U}}}{\kappa_{\beta}+l_{\mathrm{U}}} \mathrm{e}^{-l_{\mathrm{U}}(T-\tau)}\left(1-\mathrm{e}^{-\left(\kappa_{\beta}+l_{\mathrm{U}}\right) \tau}\right)\right. \\
& +\frac{m_{\mathrm{U}} n_{\mathrm{V}}}{\kappa_{\beta}+l_{\mathrm{V}}} \mathrm{e}^{-l_{\mathrm{V}}(T-\tau)}\left(1-\mathrm{e}^{-\left(\kappa_{\beta}+l_{\mathrm{V}}\right) \tau}\right) \\
& \left.+\frac{n_{\mathrm{U}} n_{\mathrm{V}}}{\kappa_{\beta}+l_{\mathrm{U}}+l_{\mathrm{V}}} \mathrm{e}^{-\left(l_{\mathrm{U}}+l_{\mathrm{V}}\right)(T-\tau)}\left(1-\mathrm{e}^{-\left(\kappa_{\beta}+l_{\mathrm{U}}+l_{\mathrm{V}}\right) \tau}\right)\right], \\
F_{2}(\tau, \omega)= & \frac{\left(\mu_{\mathrm{V}}-v(0)\right) B_{1}^{*}}{\kappa_{\eta}+\kappa_{\mathrm{V}}-\tilde{B}_{3}} \mathrm{e}^{\left(\kappa_{\mathrm{V}}-\tilde{B}_{3}\right) \tau-\kappa_{\mathrm{V}} T}+\frac{\left(v(0)-\mu_{\mathrm{V}}\right) B_{1}^{*}}{\kappa_{\eta}+\kappa_{\mathrm{V}}} \mathrm{e}^{\kappa_{\mathrm{V}}(\tau-T)} \\
& +\frac{\mu_{\mathrm{V}} B_{1}^{*}}{\kappa_{\eta}}-\frac{\mu_{\mathrm{V}} B_{1}^{*}}{\kappa_{\eta}-\tilde{B}_{3}} \mathrm{e}^{-\tilde{B}_{3} \tau}+B_{1}^{*} B_{2}^{*} \mathrm{e}^{-\kappa_{\eta} \tau}, \\
A_{2}(\tau, \omega)= & r_{\mathrm{f}} i \omega \tau+\kappa_{\mathrm{V}} \mu_{\mathrm{V}} \tilde{H}_{1}(\tau, \omega)+\left(\kappa_{\eta} \mu_{\eta}+i \omega \rho_{\mathrm{S} \eta} \sigma_{\eta} m_{\mathrm{V}}\right) \tilde{H}_{2}(\tau, \omega) \\
& +i \omega \rho_{\mathrm{S} \eta} \sigma_{\eta} n_{\mathrm{V}} \tilde{H}_{3}(\tau, \omega)+\left(\kappa_{\beta} \mu_{\beta}+i \omega \rho_{\mathrm{S} \beta} \sigma_{\beta} m_{\mathrm{V}}\right) \tilde{H}_{4}(\tau, \omega) \\
& +i \omega \rho_{\mathrm{S} \beta} \sigma_{\beta} n_{\mathrm{V}} \tilde{H}_{5}(\tau, \omega)+\frac{1}{2} \sigma_{\beta}^{2} \tilde{H}_{6}(\tau, \omega)+\frac{1}{2} \sigma_{\eta}^{2} \tilde{H}_{7}(\tau, \omega) .
\end{aligned}
$$

The $\tilde{H}$ functions in Equation (47) are defined as.

$$
\begin{aligned}
& \tilde{H}_{1}(\tau, \omega)=\int_{0}^{\tau} B_{2}(s, \omega) \mathrm{d} s, \tilde{H}_{2}(\tau, \omega)=\int_{0}^{\tau} F_{2}(s, \omega) \mathrm{d} s, \\
& \tilde{H}_{3}(\tau, \omega)=\int_{0}^{\tau} \mathrm{e}^{-l_{\mathrm{V}}(T-s)} F_{2}(s, \omega) \mathrm{d} s, \tilde{H}_{4}(\tau, \omega)=\int_{0}^{\tau} E_{2}(s, \omega) \mathrm{d} s, \\
& \tilde{H}_{5}(\tau, \omega)=\int_{0}^{\tau} \mathrm{e}^{-l_{\mathrm{V}}(T-s)} E_{2}(s, \omega) \mathrm{d} s, \tilde{H}_{6}(\tau, \omega)=\int_{0}^{\tau} E_{2}(s, \omega)^{2} \mathrm{~d} s, \\
& \tilde{H}_{7}(\tau, \omega)=\int_{0}^{\tau} F_{2}(s, \omega)^{2} \mathrm{~d} s,
\end{aligned}
$$

and the coefficients $\tilde{B}_{1}, \tilde{B}_{2}, \tilde{B}_{3}, B_{1}^{*}, B_{2}^{*}$ are respectively given by

$$
\begin{gathered}
\tilde{B}_{1}=\sqrt{\kappa_{\mathrm{V}}^{2}+\sigma_{\mathrm{V}}^{2} \omega(\omega+i)}, \\
\tilde{B}_{2}=\frac{-\kappa_{\mathrm{V}}+\tilde{B}_{1}}{\kappa_{\mathrm{V}}+\tilde{B}_{1}}, \\
\tilde{B}_{3}=-\ln \left(\frac{\mathrm{e}^{-\tilde{B}_{1}}-\tilde{B}_{2} \mathrm{e}^{-\tilde{B}_{1}}}{1-\tilde{B}_{2} \mathrm{e}^{-\tilde{B}_{1}}}\right), \\
B_{1}^{*}=\frac{i \omega}{\sigma_{\mathrm{V}}}\left(\kappa_{\mathrm{V}}-\tilde{B}_{1}\right), \\
B_{2}^{*}=-\frac{\mu_{\mathrm{V}}}{\kappa_{\mathrm{V}}}-\frac{v(0)-\mu_{\mathrm{V}}}{\kappa_{\eta}+\kappa_{\mathrm{V}}} \mathrm{e}^{-\kappa_{\mathrm{V}} T}-\frac{\mu_{\mathrm{V}}-v(0)}{\kappa_{\eta}+\kappa_{\mathrm{V}}-\tilde{B}_{3}} \mathrm{e}^{-\kappa_{\mathrm{V}} T}+\frac{\mu_{\mathrm{V}}}{\kappa_{\eta}-\tilde{B}_{3}},
\end{gathered}
$$

with $m_{\mathrm{U}}, m_{\mathrm{V}}, n_{\mathrm{U}}, n_{\mathrm{V}}, l_{\mathrm{U}}, l_{\mathrm{V}}$ as in Proposition 1 .

The proof of Lemma 2 is similar to the one for Lemma 1 and is left to the reader.

Next, we turn to the calculation of the term

$$
\mathbb{E}^{\mathbb{Q}}\left[\mathrm{e}^{-\sqrt{V(0)} \sqrt{U(0)}]_{t}^{T} \beta(s) \mathrm{d} s}\right]
$$

in (18), where $\xi=\int_{t}^{T} \beta(s) \mathrm{d} s$ is the integrated OU process. Since the OU 
process is an affine process, we can calculate the above expectation in closed-form. This is presented in the next lemma.

Lemma 3. Let

$$
\xi=\int_{t}^{T} \beta(s) \mathrm{d} s
$$

be an integrated correlation process, where $\beta(t)$ follows an OU process.

Then, we have that

$$
\mathbb{E}^{\mathbb{Q}}\left[\mathrm{e}^{-\sqrt{V(0)} \sqrt{U(0)} \int_{0}^{t} \beta(s) \mathrm{d} s}\right]=\mathrm{e}^{-\delta(t)-\beta(0) \chi(t)},
$$

with

$$
\begin{gathered}
\delta(t)=\frac{\sqrt{V(0)} \sqrt{U(0)}}{\kappa_{\beta}}\left(1-\mathrm{e}^{-\kappa_{\beta} t}\right) \\
\chi(t)=\frac{V(0) U(0) \sigma_{\beta}^{2}}{4 \kappa_{\beta}^{3}}\left(\mathrm{e}^{-2 \kappa_{\beta} t}-4 \mathrm{e}^{-\kappa_{\beta} t}+3\right)+\mu_{\beta} \frac{\sqrt{V(0)} \sqrt{U(0)}}{\kappa_{\beta}}\left(\mathrm{e}^{-\kappa_{\beta} t}-1\right) \\
+\left(\mu_{\beta} \sqrt{V(0)} \sqrt{U(0)}-\frac{V(0) U(0) \sigma_{\beta}^{2}}{2 \kappa_{\beta}^{2}}\right) t .
\end{gathered}
$$

Proof. The proof follows the same line as the corresponding result proven in [1], Lemma 3.3, with constant volatilities $\sqrt{V(0)}, \sqrt{U(0)}$ respectively.

\subsection{The Bounded Jacobi Process}

In this section, we assume that the processes $\eta(t), \gamma(t)$ and $\beta(t)$ follow a bounded Jacobi (BJ) process. In particular, the drift coefficient for a BJ process is exactly the same as the one for an OU process. What changes is the volatility coefficient, and in particular, we have that

$$
\begin{aligned}
& b(t, \eta(t))=\sigma_{\eta} \sqrt{1-\eta(t)^{2}}, \\
& d(t, \gamma(t))=\sigma_{\gamma} \sqrt{1-\gamma(t)^{2}}, \\
& g(t, \beta(t))=\sigma_{\beta} \sqrt{1-\beta(t)^{2}} .
\end{aligned}
$$

The $\mathrm{BJ}$ process is bounded to $(-1,1)^{3}$ as long as the following restriction on the model parameters holds (for the detailed derivation, see [6]):

$$
\kappa_{\rho}>\frac{\sigma_{\rho}^{2}}{1 \pm \mu_{\rho}} \text {. }
$$

Similar to what we have done in Section 4.1, we observe that the non-affine terms under the BJ stochastic correlation assumption in the pricing PDE (8) can be approximated as follows:

$$
\begin{aligned}
& \sigma_{\eta}^{2}\left(1-\eta(t)^{2}\right) \approx \sigma_{\eta}^{2}\left(1-\mathbb{E}^{\mathbb{Q}}\left[\eta(t)^{2}\right]\right), \\
& \sigma_{\gamma}^{2}\left(1-\gamma(t)^{2}\right) \approx \sigma_{\gamma}^{2}\left(1-\mathbb{E}^{\mathbb{Q}}\left[\gamma(t)^{2}\right]\right), \\
& \sigma_{\beta}^{2}\left(1-\beta(t)^{2}\right) \approx \sigma_{\beta}^{2}\left(1-\mathbb{E}^{\mathbb{Q}}\left[\beta(t)^{2}\right]\right),
\end{aligned}
$$

${ }^{3}$ It means that the boundaries -1 and 1 are not attractive and unattainable. 
and

$$
\left\{\begin{array}{l}
\rho_{\mathrm{S} \beta} \sigma_{\beta} \sqrt{V(t)} \sqrt{1-\beta(t)^{2}} \approx \rho_{\mathrm{S} \beta} \sigma_{\beta} \mathbb{E}^{\mathbb{Q}}[\sqrt{V(t)}] \mathbb{E}^{\mathbb{Q}}\left[\sqrt{1-\beta(t)^{2}}\right], \\
\rho_{\mathrm{S} \eta} \sigma_{\eta} \sqrt{V(t)} \sqrt{1-\eta(t)^{2}} \approx \rho_{\mathrm{S} \eta} \sigma_{\eta} \mathbb{E}^{\mathbb{Q}}[\sqrt{V(t)}] \mathbb{E}^{\mathbb{Q}}\left[\sqrt{1-\eta(t)^{2}}\right], \\
\rho_{\mathrm{X} \beta} \sigma_{\beta} \sqrt{U(t)} \sqrt{1-\beta(t)^{2}} \approx \rho_{\mathrm{X} \beta} \sigma_{\beta} \mathbb{E}^{\mathbb{Q}}[\sqrt{U(t)}] \mathbb{E}^{\mathbb{Q}}\left[\sqrt{1-\beta(t)^{2}}\right], \\
\rho_{\mathrm{X} \gamma} \sigma_{\gamma} \sqrt{U(t)} \sqrt{1-\gamma(t)^{2}} \approx \rho_{\mathrm{X} \gamma} \sigma_{\gamma} \mathbb{E}^{\mathbb{Q}}[\sqrt{U(t)}] \mathbb{E}^{\mathbb{Q}}\left[\sqrt{1-\gamma(t)^{2}}\right] .
\end{array}\right.
$$

Now, if we look at Equation (59), we can see that the proposed approximations involved the second moment of the BJ stochastic correlation process.

In particular, [7] has shown that, for a stochastic process $\rho(t)$ following the $\mathrm{BJ}$ process dynamics, the second moment is given by:

$$
\begin{aligned}
\mathbb{E}^{\mathbb{Q}}\left[\rho(t)^{2}\right] & \frac{\mathrm{e}^{-t\left(\sigma_{\rho}^{3}+2 \kappa_{\rho}\right)}}{\sigma_{\rho}^{4}+3 \kappa_{\rho} \sigma_{\rho}^{2}+2 \kappa_{\rho}^{2}}\left(\rho(0)^{2}\left(\sigma_{\rho}^{4}+3 \kappa_{\rho} \sigma_{\rho}^{2}+2 \kappa_{\rho}^{2}\right)\right. \\
& +2 \mu_{\rho} \kappa_{\rho} \rho(0)\left(\sigma_{\rho}^{2}+2 \kappa_{\rho}\right)\left(\mathrm{e}^{\left(\sigma_{\rho}^{2}+\kappa_{\rho}\right) t}-1\right)+\sigma_{\rho}^{2}\left(\sigma_{\rho}^{2}+\kappa_{\rho}\right)\left(\mathrm{e}^{t\left(\sigma_{\rho}^{2}+2 \kappa_{\rho}\right)}-1\right) \\
& \left.-2 \mu_{\rho}^{2} \kappa_{\rho}\left(\kappa_{\rho}\left(2 \mathrm{e}^{t\left(\sigma_{\rho}^{2}+\kappa_{\rho}\right)}-\mathrm{e}^{t\left(\sigma_{\rho}^{2}+2 \kappa_{\rho}\right)}-1\right)-\sigma_{\rho}^{2} \mathrm{e}^{t\left(\sigma_{\rho}^{2}+\kappa_{\rho}\right)}\left(\mathrm{e}^{t \kappa_{\rho}}-1\right)\right)\right) .
\end{aligned}
$$

As Equation (61) is rather complicated and not convenient for further calculations, we rely instead on an approximation, which has been proposed in [2], and used as well in [1]. The result is reported in the following proposition.

Proposition 2 (3.2 in [2] or alternatively 3.1 in [1]) Let $\rho(t)$ a stochastic process, whose dynamics is given by:

$$
\mathrm{d} \rho(t)=\kappa_{\rho}\left(\mu_{\rho}-\rho(t)\right) \mathrm{d} t+\sigma_{\rho} \sqrt{1-\rho(t)^{2}} \mathrm{~d} W_{\rho}(t)^{\mathbb{Q}} .
$$

Then, the function $h_{\rho}(t) \equiv \mathbb{E}^{\mathbb{Q}}\left[\rho(t)^{2}\right]$ can be approximated by:

$$
\mathbb{E}^{\mathbb{Q}}\left[\rho(t)^{2}\right] \approx \mathrm{e}^{-s} \rho^{t}+r_{\rho} \mathrm{e}^{-p_{\rho} t}+q_{\rho},
$$

where

$$
\begin{gathered}
q_{\rho}=\frac{\left(\sigma_{\rho}^{2}+\kappa_{\rho}\right)\left(\sigma_{\rho}^{2}+2 \kappa_{\rho} \mu_{\rho}^{2}\right)}{\sigma_{\rho}^{4}+3 \kappa_{\rho} \sigma_{\rho}^{2}+2 \kappa_{\rho}^{2}}, r_{\rho}=\rho(0)^{2}-q_{\rho}-1, \\
s_{\rho}=-2 \ln \left(\alpha_{\rho}-r_{\rho} \mathrm{e}^{-\frac{p_{\rho}}{2}}\right), p_{\rho}=-2 \ln \left(\frac{r_{\rho} \alpha_{\rho}-\sqrt{r_{\rho}^{2} \alpha_{\rho}^{2}-\theta_{\rho} \psi_{\rho}}}{\theta_{\rho}}\right),
\end{gathered}
$$

with

$$
\alpha_{\rho}=h_{\rho}(0.5)-q_{\rho}, \theta_{\rho}=r_{\rho}\left(1+r_{\rho}\right), \psi_{\rho}=\alpha_{\rho}^{2}+q_{\rho}-h_{\rho}(1) .
$$

Proof. See Appendix B.2 in [2]. 
Finally, if we look at Equation (60), the only remaining quantity we need to calculate is $\mathbb{E}^{\mathbb{Q}}\left[\sqrt{1-\rho(t)^{2}}\right]$, where $\rho \in\{\eta, \gamma, \beta\}$. In order to calculate this expectation, we state the result in [2], where it is shown that

$$
\begin{aligned}
\mathbb{E}^{\mathbb{Q}}\left[\sqrt{1-\rho(t)^{2}}\right] & =\sqrt{\mathbb{E}^{\mathbb{Q}}\left[1-\rho(t)^{2}\right]-\frac{\mathbb{E}^{\mathbb{Q}}[\rho(t)]^{2}}{1-\mathbb{E}^{\mathbb{Q}}[\rho(t)]^{2}} \operatorname{Var}^{\mathbb{Q}}[\rho(t)]} \\
& =\sqrt{1-\frac{\mathbb{E}^{\mathbb{Q}}\left[\rho(t)^{2}\right]-\mathbb{E}^{\mathbb{Q}}[\rho(t)]^{4}}{1-\mathbb{E}^{\mathbb{Q}}[\rho(t)]^{2}}} .
\end{aligned}
$$

The next proposition contains the relevant approximation.

Proposition 3 (3.3 in [2] or alternatively 3.2 in [1]) Let $\rho(t)$ a stochastic process, whose dynamics is given by:

$$
\mathrm{d} \rho(t)=\kappa_{\rho}\left(\mu_{\rho}-\rho(t)\right) \mathrm{d} t+\sigma_{\rho} \sqrt{1-\rho(t)^{2}} \mathrm{~d} W_{\rho}(t)^{\mathbb{Q}} .
$$

Then, the function $\hat{h}_{\rho}(t) \equiv \mathbb{E}^{\mathbb{Q}}\left[\sqrt{1-\rho(t)^{2}}\right]$ can be approximated by.

$$
\mathbb{E}^{\mathbb{Q}}\left[\sqrt{1-\rho(t)^{2}}\right] \approx \mathrm{e}^{-\hat{s}_{\rho} t}+\hat{r}_{\rho} \mathrm{e}^{-\hat{p}_{\rho} t}+\hat{q}_{\rho},
$$

where

$$
\begin{gathered}
\hat{q}_{\rho}=\sqrt{1-\frac{\left(\sigma_{\rho}^{2}+\kappa_{\rho}\right)\left(\sigma_{\rho}^{2}+2 \kappa_{\rho} \mu_{\rho}^{2}\right)-\mu_{\rho}^{4}\left(\sigma_{\rho}^{4}+3 \kappa_{\rho} \sigma_{\rho}^{2}+2 \kappa_{\rho}^{2}\right)}{\left(1-\mu_{\rho}^{2}\right)\left(\sigma_{\rho}^{4}+3 \kappa_{\rho} \sigma_{\rho}^{2}+2 \kappa_{\rho}^{2}\right)},} \\
\hat{r}_{\rho}=\sqrt{1-\rho(0)^{2}}-\hat{q}_{\rho}-1, \hat{s}_{\rho}=-2 \ln \left(\hat{\alpha}_{\rho}-\hat{r}_{\rho} \mathrm{e}^{-\frac{\hat{p}_{\rho}}{2}}\right), \\
\hat{p}_{\rho}=-2 \ln \left(\frac{\hat{r}_{\rho} \hat{\alpha}_{\rho}-\sqrt{\hat{r}_{\rho}^{2} \hat{\alpha}_{\rho}^{2}-\hat{\theta}_{\rho} \hat{\psi}_{\rho}}}{\hat{\theta}_{\rho}}\right),
\end{gathered}
$$

with

$$
\hat{\alpha}_{\rho}=\hat{h}_{\rho}(0.5)-\hat{q}_{\rho}, \hat{\theta}_{\rho}=\hat{r}_{\rho}\left(1+\hat{r}_{\rho}\right), \hat{\psi}_{\rho}=\hat{\alpha}_{\rho}^{2}+\hat{q}_{\rho}-\hat{h}_{\rho}(1) .
$$

Proof. See Appendix B.3 in [2].

We have therefore all the elements in order to find a solution for the pricing PDE (8), under the assumption that the correlation processes follow a bounded Jacobi process.

First, we observe that Equation (8) becomes

$$
\begin{aligned}
& \frac{\partial P}{\partial t}+\left(r_{\mathrm{f}}-\frac{1}{2} V(t)-\beta(t) \mathbb{E}^{\mathbb{Q}}[\sqrt{V(t)}] \mathbb{E}^{\mathbb{Q}}[\sqrt{U(t)}]\right) \frac{\partial P}{\partial y} \\
& +\left(r_{\mathrm{d}}-r_{\mathrm{f}}-\frac{1}{2} U(t)\right) \frac{\partial P}{\partial z}+\kappa_{\eta}\left(\mu_{\eta}-\eta(t)\right) \frac{\partial P}{\partial \eta}+\kappa_{\mathrm{V}}\left(\mu_{\mathrm{V}}-V(t)\right) \frac{\partial P}{\partial v} \\
& +\kappa_{\mathrm{U}}\left(\mu_{\mathrm{U}}-U(t)\right) \frac{\partial P}{\partial u}+\kappa_{\gamma}\left(\mu_{\gamma}-\gamma(t)\right) \frac{\partial P}{\partial \gamma}+\kappa_{\beta}\left(\mu_{\beta}-\beta(t)\right) \frac{\partial P}{\partial \beta}
\end{aligned}
$$




$$
\begin{aligned}
& +\frac{1}{2} V(t) \frac{\partial^{2} P}{\partial y^{2}}+\frac{1}{2} U(t) \frac{\partial^{2} P}{\partial z^{2}}+\frac{1}{2} \sigma_{\mathrm{V}}^{2} V(t) \frac{\partial^{2} P}{\partial v^{2}}+\frac{1}{2} \sigma_{\mathrm{U}}^{2} U(t) \frac{\partial^{2} P}{\partial u^{2}} \\
& +\frac{1}{2} \sigma_{\eta}^{2}\left(1-\mathbb{E}^{\mathbb{Q}}\left[\eta(t)^{2}\right]\right) \frac{\partial^{2} P}{\partial \eta^{2}}+\frac{1}{2} \sigma_{\gamma}^{2}\left(1-\mathbb{E}^{\mathbb{Q}}\left[\gamma(t)^{2}\right]\right) \frac{\partial^{2} P}{\partial \gamma^{2}} \\
& +\frac{1}{2} \sigma_{\beta}^{2}\left(1-\mathbb{E}^{\mathbb{Q}}\left[\beta(t)^{2}\right]\right) \frac{\partial^{2} P}{\partial \beta^{2}}+\beta(t) \mathbb{E}^{\mathbb{Q}}[\sqrt{V(t)}] \mathbb{E}^{\mathbb{Q}}[\sqrt{U(t)}] \frac{\partial^{2} P}{\partial y \partial z} \\
& +\sigma_{\mathrm{V}} \mathbb{E}^{\mathbb{Q}}[V(t)] \eta(t) \frac{\partial^{2} P}{\partial y \partial v}+\sigma_{\mathrm{U}} \mathbb{E}^{\mathbb{Q}}[U(t)] \gamma(t) \frac{\partial^{2} P}{\partial z \partial u} \\
& +\rho_{\mathrm{S} \beta} \sigma_{\beta} \mathbb{E}^{\mathbb{Q}}[\sqrt{V(t)}] \mathbb{E}^{\mathbb{Q}}\left[\sqrt{1-\beta(t)^{2}}\right] \frac{\partial^{2} P}{\partial y \partial \beta} \\
& +\rho_{\mathrm{S} \eta} \sigma_{\eta} \mathbb{E}^{\mathbb{Q}}[\sqrt{V(t)}] \mathbb{E}^{\mathbb{Q}}\left[\sqrt{1-\eta(t)^{2}}\right] \frac{\partial^{2} P}{\partial y \partial \eta} \\
& +\rho_{\mathrm{X} \beta} \sigma_{\beta} \mathbb{E}^{\mathbb{Q}}[\sqrt{U(t)}] \mathbb{E}^{\mathbb{Q}}\left[\sqrt{1-\beta(t)^{2}}\right] \frac{\partial^{2} P}{\partial z \partial \beta} \\
& +\rho_{\mathrm{X} \gamma} \sigma_{\gamma} E^{\mathbb{Q}}[\sqrt{U(t)}] \mathbb{E}^{\mathbb{Q}}\left[\sqrt{1-\gamma(t)^{2}}\right] \frac{\partial^{2} P}{\partial z \partial \gamma}-r_{\mathrm{d}} P=0 .
\end{aligned}
$$

By substituting (18) into the pricing PDE (74), we obtain the following PDEs in $P_{1}$ and $P_{2}$ respectively:

$$
\begin{aligned}
& \frac{\partial P_{1}}{\partial t}+\left(r_{\mathrm{f}}+\frac{1}{2} V(t)-\beta(t) \mathbb{E}[\sqrt{V(t)}] \mathbb{E}[\sqrt{U(t)}]\right) \frac{\partial P_{1}}{\partial y} \\
& +\left(r_{\mathrm{d}}-r_{\mathrm{f}}-\frac{1}{2} U(t)+\beta(t) \mathbb{E}[\sqrt{V(t)}] \mathbb{E}[\sqrt{U(t)}]\right) \frac{\partial P_{1}}{\partial z} \\
& +\left(\kappa_{\eta}\left(\mu_{\eta}-\eta(t)\right)+\rho_{\mathrm{S} \eta} \sigma_{\eta} \mathbb{E}[\sqrt{V(t)}] \mathbb{E}\left[\sqrt{1-\eta(t)^{2}}\right]\right) \frac{\partial P_{1}}{\partial \eta} \\
& +\left(\kappa_{\mathrm{V}}\left(\mu_{\mathrm{V}}-V(t)\right)+\sigma_{\mathrm{V}} \mathbb{E}[V(t)] \eta(t)\right) \frac{\partial P_{1}}{\partial v}+\kappa_{\mathrm{U}}\left(\mu_{\mathrm{U}}-U(t)\right) \frac{\partial P_{1}}{\partial u} \\
& +\left(\kappa_{\beta}\left(\mu_{\beta}-\beta(t)\right)+\rho_{\mathrm{S} \beta} \sigma_{\beta} \mathbb{E}[\sqrt{V(t)}]\left[\sqrt{1-\beta(t)^{2}}\right]\right) \frac{\partial P_{1}}{\partial \beta} \\
& +\kappa_{\gamma}\left(\mu_{\gamma}-\gamma(t)\right) \frac{\partial P_{1}}{\partial \gamma}+\frac{1}{2} V(t) \frac{\partial^{2} P_{1}}{\partial y^{2}}+\frac{1}{2} U(t) \frac{\partial^{2} P_{1}}{\partial z^{2}}+\frac{1}{2} \sigma_{\mathrm{v}}^{2} V(t) \frac{\partial^{2} P_{1}}{\partial v^{2}} \\
& +\frac{1}{2} \sigma_{\mathrm{U}}^{2} U(t) \frac{\partial^{2} P_{1}}{\partial u^{2}}+\frac{1}{2} \sigma_{\beta}^{2}\left(1-\mathbb{E}\left[\beta(t)^{2}\right]\right) \frac{\partial^{2} P_{1}}{\partial \beta^{2}}+\frac{1}{2} \sigma_{\eta}^{2}\left(1-\mathbb{E}\left[\eta(t)^{2}\right]\right) \frac{\partial^{2} P_{1}}{\partial \eta^{2}} \\
& +\frac{1}{2} \sigma_{\gamma}^{2}\left(1-\mathbb{E}\left[\gamma(t)^{2}\right]\right) \frac{\partial^{2} P_{1}}{\partial \gamma^{2}}+\beta(t) \mathbb{E}[\sqrt{V(t)}] \mathbb{E}[\sqrt{U(t)}] \frac{\partial^{2} P_{1}}{\partial y \partial z} \\
& +\sigma_{\mathrm{v}} \mathbb{E}[V(t)] \eta(t) \frac{\partial^{2} P_{1}}{\partial y \partial v}+\sigma_{\mathrm{U}} \mathbb{E}[U(t)] \gamma(t) \frac{\partial^{2} P_{1}}{\partial z \partial u} \\
& +\rho_{\mathrm{S} \beta} \sigma_{\beta} \mathbb{E}[\sqrt{V(t)}] \mathbb{E}\left[\sqrt{1-\beta(t)^{2}}\right] \frac{\partial^{2} P_{1}}{\partial \beta \partial y} \\
& +\rho_{\mathrm{S} \eta} \sigma_{\eta} \mathbb{E}[\sqrt{V(t)}] \mathbb{E}\left[\sqrt{1-\eta(t)^{2}}\right] \frac{\partial^{2} P_{1}}{\partial \eta \partial y} \\
& +\rho_{\mathrm{X} \beta} \sigma_{\beta} \mathbb{E}[\sqrt{U(t)}] \mathbb{E}\left[\sqrt{1-\beta(t)^{2}}\right] \frac{\partial^{2} P_{1}}{\partial z \partial \beta} \\
& +\rho_{\mathrm{X} \gamma} \sigma_{\gamma} \mathbb{E}[\sqrt{U(t)}] \mathbb{E}\left[\sqrt{1-\gamma(t)^{2}}\right] \frac{\partial^{2} P_{1}}{\partial z \partial \gamma}=0,
\end{aligned}
$$


and

$$
\begin{aligned}
& \frac{\partial P_{2}}{\partial t}+\left(r_{\mathrm{f}}-\frac{1}{2} V(t)-\beta(t) \mathbb{E}[\sqrt{V(t)}] \mathbb{E}[\sqrt{U(t)}]\right) \frac{\partial P_{2}}{\partial y} \\
& +\left(r_{\mathrm{d}}-r_{\mathrm{f}}-\frac{1}{2} U(t)\right) \frac{\partial P_{2}}{\partial z}+\kappa_{\eta}\left(\mu_{\eta}-\eta(t)\right) \frac{\partial P_{2}}{\partial \eta}+\kappa_{\mathrm{V}}\left(\mu_{\mathrm{V}}-V(t)\right) \frac{\partial P_{2}}{\partial v} \\
& +\kappa_{\mathrm{U}}\left(\mu_{\mathrm{U}}-U(t)\right) \frac{\partial P_{2}}{\partial u}+\kappa_{\beta}\left(\mu_{\beta}-\beta(t)\right) \frac{\partial P_{2}}{\partial \beta}+\kappa_{\gamma}\left(\mu_{\gamma}-\gamma(t)\right) \frac{\partial P_{2}}{\partial \gamma} \\
& +\frac{1}{2} V(t) \frac{\partial^{2} P_{2}}{\partial y^{2}}+\frac{1}{2} U(t) \frac{\partial^{2} P_{2}}{\partial z^{2}}+\frac{1}{2} \sigma_{\mathrm{V}}^{2} V(t) \frac{\partial^{2} P_{2}}{\partial v^{2}}+\frac{1}{2} \sigma_{\mathrm{U}}^{2} U(t) \frac{\partial^{2} P_{2}}{\partial u^{2}} \\
& +\frac{1}{2} \sigma_{\beta}^{2}\left(1-\mathbb{E}\left[\beta(t)^{2}\right]\right) \frac{\partial^{2} P_{2}}{\partial \beta^{2}}+\frac{1}{2} \sigma_{\eta}^{2}\left(1-\mathbb{E}\left[\eta(t)^{2}\right]\right) \frac{\partial^{2} P_{2}}{\partial \eta^{2}} \\
& +\frac{1}{2} \sigma_{\gamma}^{2}\left(1-\mathbb{E}\left[\gamma(t)^{2}\right]\right) \frac{\partial^{2} P_{2}}{\partial \gamma^{2}}+\beta(t) \mathbb{E}[\sqrt{V(t)}] \mathbb{E}[\sqrt{U(t)}] \frac{\partial^{2} P_{2}}{\partial y \partial z} \\
& +\sigma_{\mathrm{V}} \mathbb{E}[V(t)] \eta(t) \frac{\partial^{2} P_{2}}{\partial y \partial v}+\sigma_{\mathrm{U}} \mathbb{E}[U(t)] \gamma(t) \frac{\partial^{2} P_{2}}{\partial z \partial u} \\
& +\rho_{\mathrm{S} \beta} \sigma_{\beta} \mathbb{E}[\sqrt{V(t)}] \mathbb{E}\left[\sqrt{1-\beta(t)^{2}}\right] \frac{\partial^{2} P_{2}}{\partial \beta \partial y} \\
& +\rho_{\mathrm{S} \eta} \sigma_{\eta} \mathbb{E}[\sqrt{V(t)}] \mathbb{E}\left[\sqrt{1-\eta(t)^{2}}\right] \frac{\partial^{2} P_{2}}{\partial \eta \partial y} \\
& +\rho_{\mathrm{X} \beta} \sigma_{\beta} \mathbb{E}[\sqrt{U(t)}] \mathbb{E}\left[\sqrt{1-\beta(t)^{2}}\right] \frac{\partial^{2} P_{2}}{\partial z \partial \beta} \\
& +\rho_{\mathrm{X} \gamma} \sigma_{\gamma} \mathbb{E}[\sqrt{U(t)}] \mathbb{E}\left[\sqrt{1-\gamma(t)^{2}}\right] \frac{\partial^{2} P_{2}}{\partial z \partial \gamma}=0 .
\end{aligned}
$$

As pointed out in the previous section, we know that the corresponding characteristic functions $\phi=\phi_{j}(t, y, z, v, u, \beta, \eta, \gamma ; \omega)$ of $P_{j}, j=1,2$, must also satisfy the PDEs (75) and (76), respectively. Their solutions can be found in a closed-form as stated in the next lemmas.

Lemma 4. The characteristic function of $P_{1}$ in (18), under the assumption that the correlation processes $\beta(t), \eta(t)$ and $\gamma(t)$ follow a BJ process, is given by:

$$
\begin{aligned}
\phi_{1}^{\mathrm{BJ}}= & \exp \left(\tilde{A}_{1}(\tau, \omega)+B_{1}(\tau, \omega) v(t)+C_{1}(\tau, \omega) u(t)+D_{1}(\tau, \omega) z(t)\right. \\
& \left.+E_{1}(\tau, \omega) \beta(t)+F_{1}(\tau, \omega) \eta(t)+G_{1}(\tau, \omega) \gamma(t)+i \omega y(t)\right),
\end{aligned}
$$

where $B_{1}, C_{1}, D_{1}, E_{1}, F_{1}$ and $G_{1}$ are as in Lemma 1 , and where $\tilde{A}_{1}$ is given by:

$$
\begin{aligned}
\tilde{A}_{1}(\tau, \omega)= & r_{\mathrm{f}} i \omega \tau+\kappa_{\mathrm{V}} \mu_{\mathrm{V}} H_{1}(\tau, \omega)+\left(\kappa_{\eta} \mu_{\eta}+(i \omega+1) \rho_{\mathrm{S} \eta} \sigma_{\eta} m_{\mathrm{V}} \hat{q}_{\eta}\right) H_{2}(\tau, \omega) \\
& +(i \omega+1) \rho_{\mathrm{S} \eta} \sigma_{\eta}\left(m_{\mathrm{V}} H_{3}(\tau, \omega)+m_{\mathrm{V}} \hat{r}_{\eta} H_{4}(\tau, \omega)\right. \\
& \left.+n_{\mathrm{V}} H_{5}(\tau, \omega)+n_{\mathrm{V}} \hat{r}_{\eta} H_{6}(\tau, \omega)+n_{\mathrm{V}} \hat{q}_{\eta} H_{7}(\tau, \omega)\right) \\
& +\left(\kappa_{\beta} \mu_{\beta}+(i \omega+1) \rho_{\mathrm{S} \beta} \sigma_{\beta} m_{\mathrm{V}} \hat{q}_{\beta}\right) H_{8}(\tau, \omega) \\
& +(i \omega+1) \rho_{\mathrm{S} \beta} \sigma_{\beta}\left(m_{\mathrm{V}} H_{9}(\tau, \omega)+m_{\mathrm{V}} \hat{r}_{\beta} H_{10}(\tau, \omega)+n_{\mathrm{V}} H_{11}(\tau, \omega)\right.
\end{aligned}
$$




$$
\begin{aligned}
& \left.+n_{\mathrm{V}} \hat{r}_{\beta} H_{12}(\tau, \omega)+n_{\mathrm{v}} \hat{q}_{\beta} H_{13}(\tau, \omega)\right)+\frac{1}{2}\left(\sigma_{\beta}^{2}\left(1-q_{\beta}\right) H_{14}(\tau, \omega)\right. \\
& \left.+\sigma_{\eta}^{2}\left(1-q_{\eta}\right) H_{15}(\tau, \omega)\right)-\frac{1}{2}\left(\sigma_{\beta}^{2}\left(H_{16}(\tau, \omega)+r_{\beta} H_{17}(\tau, \omega)\right)\right. \\
& \left.+\sigma_{\eta}^{2}\left(H_{18}(\tau, \omega)+r_{\eta} H_{19}(\tau, \omega)\right)\right) .
\end{aligned}
$$

The H functions in Equation (78) are defined as.

$$
\begin{aligned}
& H_{1}(\tau, \omega)=\int_{0}^{\tau} B_{1}(s, \omega) \mathrm{d} s, H_{2}(\tau, \omega)=\int_{0}^{\tau} F_{1}(s, \omega) \mathrm{d} s, \\
& H_{3}(\tau, \omega)=\hat{H}\left(\tau, \omega, \hat{s}_{\eta}\right), H_{4}(\tau, \omega)=\hat{H}\left(\tau, \omega, \hat{p}_{\eta}\right), \\
& H_{5}(\tau, \omega)=\hat{H}\left(\tau, \omega, l_{\mathrm{V}}+\hat{s}_{\eta}\right), H_{6}(\tau, \omega)=\hat{H}\left(\tau, \omega, l_{\mathrm{V}}+\hat{p}_{\eta}\right), \\
& H_{7}(\tau, \omega)=\hat{H}\left(\tau, \omega, l_{\mathrm{V}}\right), H_{8}(\tau, \omega)=\int_{0}^{\tau} E_{1}(s, \omega) \mathrm{d} s, \\
& H_{9}(\tau, \omega)=\bar{H}\left(\tau, \omega, \hat{s}_{\beta}\right), H_{10}(\tau, \omega)=\bar{H}\left(\tau, \omega, \hat{p}_{\beta}\right), \\
& H_{11}(\tau, \omega)=\bar{H}\left(\tau, \omega, l_{\mathrm{V}}+\hat{s}_{\beta}\right), H_{12}(\tau, \omega)=\bar{H}\left(\tau, \omega, l_{\mathrm{V}}+\hat{p}_{\beta}\right), \\
& H_{13}(\tau, \omega)=\bar{H}\left(\tau, \omega, l_{\mathrm{V}}\right), H_{14}(\tau, \omega)=\int_{0}^{\tau} E_{1}(s, \omega)^{2} \mathrm{~d} s, \\
& H_{15}(\tau, \omega)=\int_{0}^{\tau} F_{1}(s, \omega)^{2} \mathrm{~d} s, H_{16}(\tau, \omega)=H^{\star}\left(\tau, \omega, s_{\beta}\right), \\
& H_{17}(\tau, \omega)=H^{\star}\left(\tau, \omega, p_{\beta}\right), H_{18}(\tau, \omega)=H^{\circ}\left(\tau, \omega, s_{\eta}\right), \\
& H_{19}(\tau, \omega)=H^{\circ}\left(\tau, \omega, p_{\eta}\right),
\end{aligned}
$$

where the functions $\hat{H}, \bar{H}, H^{\star}$ and $H^{\circ}$ are given by:

$$
\begin{aligned}
& \hat{H}(\tau, \omega, a)=\int_{0}^{\tau} \mathrm{e}^{-a(T-s)} F_{1}(s, \omega) \mathrm{d} s, \bar{H}(\tau, \omega, a)=\int_{0}^{\tau} \mathrm{e}^{-a(T-s)} E_{1}(s, \omega) \mathrm{d} s, \\
& H^{\star}(\tau, \omega, a)=\int_{0}^{\tau} \mathrm{e}^{-a(T-s)} E_{1}^{2}(s, \omega) \mathrm{d} s, H^{\circ}(\tau, \omega, a)=\int_{0}^{\tau} \mathrm{e}^{-a(T-s)} F_{1}^{2}(s, \omega) \mathrm{d} s,
\end{aligned}
$$

and all the other parameters have been already defined in Lemma 1.

Proof. See Appendix A.3.

Lemma 5. The characteristic function of $P_{2}$ in (18), under the assumption that the correlation processes $\beta(t), \eta(t)$ and $\gamma(t)$ follow an BJ process, is given by:

$$
\begin{aligned}
\phi_{2}^{\mathrm{BJ}}= & \exp \left(\tilde{A}_{2}(\tau, \omega)+B_{2}(\tau, \omega) v(t)+C_{2}(\tau, \omega) u(t)+D_{2}(\tau, \omega) z(t)\right. \\
& \left.+E_{2}(\tau, \omega) \beta(t)+F_{2}(\tau, \omega) \eta(t)+G_{2}(\tau, \omega) \gamma(t)+i \omega y(t)\right),
\end{aligned}
$$

where $B_{2}, C_{2}, D_{2}, E_{2}, F_{2}$ and $G_{2}$ are as in Lemma 2, and where $\tilde{A}_{2}$ is given by:

$$
\begin{aligned}
\tilde{A}_{2}(\tau, \omega)= & r_{\mathrm{f}} i \omega \tau+\kappa_{\mathrm{V}} \mu_{\mathrm{V}} \tilde{H}_{1}(\tau, \omega)+\left(\kappa_{\eta} \mu_{\eta}+i \omega \rho_{\mathrm{S} \eta} \sigma_{\eta} m_{\mathrm{v}} \hat{q}_{\eta}\right) \tilde{H}_{2}(\tau, \omega) \\
& +i \omega \rho_{\mathrm{S} \eta} \sigma_{\eta}\left(m_{\mathrm{V}} \tilde{H}_{3}(\tau, \omega)+m_{\mathrm{V}} \hat{r}_{\eta} \tilde{H}_{4}(\tau, \omega)\right. \\
& \left.+n_{\mathrm{V}} \tilde{H}_{5}(\tau, \omega)+n_{\mathrm{V}} \hat{r}_{\eta} \tilde{H}_{6}(\tau, \omega)+n_{\mathrm{V}} \hat{q}_{\eta} \tilde{H}_{7}(\tau, \omega)\right) \\
& +\left(\kappa_{\beta} \mu_{\beta}+i \omega \rho_{\mathrm{S} \beta} \sigma_{\beta} m_{\mathrm{V}} \hat{q}_{\beta}\right) \tilde{H}_{8}(\tau, \omega) \\
& +i \omega \rho_{\mathrm{S} \beta} \sigma_{\beta}\left(m_{\mathrm{V}} \tilde{H}_{9}(\tau, \omega)+m_{\mathrm{V}} \hat{r}_{\beta} \tilde{H}_{10}(\tau, \omega)+n_{\mathrm{V}} \tilde{H}_{11}(\tau, \omega)\right.
\end{aligned}
$$




$$
\begin{aligned}
& \left.+n_{\mathrm{V}} \hat{r}_{\beta} \tilde{H}_{12}(\tau, \omega)+n_{\mathrm{v}} \hat{q}_{\beta} \tilde{H}_{13}(\tau, \omega)\right)+\frac{1}{2}\left(\sigma_{\beta}^{2}\left(1-q_{\beta}\right) \tilde{H}_{14}(\tau, \omega)\right. \\
& \left.+\sigma_{\eta}^{2}\left(1-q_{\eta}\right) \tilde{H}_{15}(\tau, \omega)\right)-\frac{1}{2}\left(\sigma_{\beta}^{2}\left(\tilde{H}_{16}(\tau, \omega)+r_{\beta} \tilde{H}_{17}(\tau, \omega)\right)\right. \\
& \left.+\sigma_{\eta}^{2}\left(\tilde{H}_{18}(\tau, \omega)+r_{\eta} \tilde{H}_{19}(\tau, \omega)\right)\right) .
\end{aligned}
$$

The $\tilde{H}$ functions in Equation (82) are defined as:

$$
\begin{aligned}
& \tilde{H}_{1}(\tau, \omega)=\int_{0}^{\tau} B_{2}(s, \omega) \mathrm{d} s, \tilde{H}_{2}(\tau, \omega)=\int_{0}^{\tau} F_{2}(s, \omega) \mathrm{d} s \\
& \tilde{H}_{3}(\tau, \omega)=\hat{K}\left(\tau, \omega, \hat{s}_{\eta}\right), \tilde{H}_{4}(\tau, \omega)=\hat{K}\left(\tau, \omega, \hat{p}_{\eta}\right) \\
& \tilde{H}_{5}(\tau, \omega)=\hat{K}\left(\tau, \omega, l_{\mathrm{V}}+\hat{s}_{\eta}\right), \tilde{H}_{6}(\tau, \omega)=\hat{K}\left(\tau, \omega, l_{\mathrm{V}}+\hat{p}_{\eta}\right), \\
& \tilde{H}_{7}(\tau, \omega)=\hat{K}\left(\tau, \omega, l_{\mathrm{v}}\right), \tilde{H}_{8}(\tau, \omega)=\int_{0}^{\tau} E_{2}(s, \omega) \mathrm{d} s, \\
& \tilde{H}_{9}(\tau, \omega)=\bar{K}\left(\tau, \omega, \hat{s}_{\beta}\right), \tilde{H}_{10}(\tau, \omega)=\bar{K}\left(\tau, \omega, \hat{p}_{\beta}\right) \\
& \tilde{H}_{11}(\tau, \omega)=\bar{K}\left(\tau, \omega, l_{\mathrm{V}}+\hat{s}_{\beta}\right), \tilde{H}_{12}(\tau, \omega)=\bar{K}\left(\tau, \omega, l_{\mathrm{V}}+\hat{p}_{\beta}\right), \\
& \tilde{H}_{13}(\tau, \omega)=\bar{K}\left(\tau, \omega, l_{\mathrm{V}}\right), \tilde{H}_{14}(\tau, \omega)=\int_{0}^{\tau} E_{2}(s, \omega)^{2} \mathrm{~d} s, \\
& \tilde{H}_{15}(\tau, \omega)=\int_{0}^{\tau} F_{2}(s, \omega)^{2} \mathrm{~d} s, \tilde{H}_{16}(\tau, \omega)=K^{\star}\left(\tau, \omega, s_{\beta}\right) \\
& \tilde{H}_{17}(\tau, \omega)=K^{\star}\left(\tau, \omega, p_{\beta}\right), \tilde{H}_{18}(\tau, \omega)=K^{\circ}\left(\tau, \omega, s_{\eta}\right) \\
& \tilde{H}_{19}(\tau, \omega)=K^{\circ}\left(\tau, \omega, p_{\eta}\right),
\end{aligned}
$$

where the functions $\hat{K}, \bar{K}, K^{\star}$ and $K^{\circ}$ are given by.

$$
\begin{aligned}
& \hat{K}(\tau, \omega, a)=\int_{0}^{\tau} \mathrm{e}^{-a(T-s)} F_{2}(s, \omega) \mathrm{d} s, \bar{K}(\tau, \omega, a)=\int_{0}^{\tau} \mathrm{e}^{-a(T-s)} E_{2}(s, \omega) \mathrm{d} s, \\
& K^{\star}(\tau, \omega, a)=\int_{0}^{\tau} \mathrm{e}^{-a(T-s)} E_{2}^{2}(s, \omega) \mathrm{d} s, K^{\circ}(\tau, \omega, a)=\int_{0}^{\tau} \mathrm{e}^{-a(T-s)} F_{2}^{2}(s, \omega) \mathrm{d} s,
\end{aligned}
$$

and where all the other parameters have been already defined in Lemma 2.

The proof of Lemma 5 is similar to the one for Lemma 4 and is left to the reader.

Lemma 6. Let

$$
\xi=\int_{t}^{T} \beta(s) \mathrm{d} s
$$

be an integrated correlation process, where $\beta(t)$ follows a BJ process.

Then, we have that

$$
\mathbb{E}^{\mathbb{Q}}\left[\mathrm{e}^{-\sqrt{V(0)} \sqrt{U(0)} \int_{0}^{t} \beta(s) \mathrm{d} s}\right]=\mathrm{e}^{-\tilde{\delta}(t)-\beta(0) \tilde{\chi}(t)},
$$

with

$$
\begin{gathered}
\tilde{\delta}(t)=\frac{\sqrt{V(0)} \sqrt{U(0)}}{\kappa_{\beta}}\left(1-\mathrm{e}^{-\kappa_{\beta} t}\right) \\
\tilde{\chi}(t)=\frac{V(0) U(0) \sigma_{\beta}^{2}}{2 \kappa_{\beta}^{2}}\left(\frac{2\left(\mathrm{e}^{-\left(\kappa_{\beta}+s_{\beta}\right) t}-1\right)}{\kappa_{\beta}+s_{\beta}}+\frac{2 r_{\beta}\left(\mathrm{e}^{-\left(\kappa_{\beta}+p_{\beta}\right) t}-1\right)}{\kappa_{\beta}+p_{\beta}}\right. \\
-\frac{\mathrm{e}^{\left(2 \kappa_{\beta}+s_{\beta}\right) t}-1}{2 \kappa_{\beta}+s_{\beta}}-\frac{r_{\beta}\left(\mathrm{e}^{-\left(2 \kappa_{\beta}+p_{\beta}\right) t}-1\right)}{2 \kappa_{\beta}+p_{\beta}}
\end{gathered}
$$




$$
\begin{aligned}
& +\frac{2\left(q_{\beta}-1\right)\left(\mathrm{e}^{-\kappa_{\beta} t}-1\right)}{\kappa_{\beta}}-\frac{\left(q_{\beta}-1\right)\left(\mathrm{e}^{-2 \kappa_{\beta} t}-1\right)}{2 \kappa_{\beta}} \\
& \left.-\frac{\mathrm{e}^{-s_{\beta} t}-1}{s_{\beta}}-\frac{r_{\beta}\left(\mathrm{e}^{-p_{\beta} t}-1\right)}{p_{\beta}}+\left(q_{\beta}-1\right) t\right) \\
& +\frac{\sqrt{V(0)} \sqrt{U(0)} t}{\kappa_{\beta}}+\frac{\sqrt{U(0)} \sqrt{V(0)}\left(\mathrm{e}^{-\kappa_{\beta} t}-1\right)}{\kappa_{\beta}^{2}},
\end{aligned}
$$

where $q, r, s, p$ have been defined in Proposition 2.

\section{Monte Carlo Model Simulation: A Numerical Scheme}

In this section, we discuss how to simulate the paths for the model in Equation (7) in order to compute the price of FX quanto options applying Monte Carlo simulation. In particular, we need to generate random paths of $(U(t), V(t), \eta(t), \gamma(t), \beta(t), Z(t), Y(t))$ for all $t \in\left\{t_{i}\right\}_{i=1}^{N} \equiv \mathcal{T}^{4}$.

To be more precise, for an arbitrary time increment $\Delta$, we need to generate a random sample of

$(U(t+\Delta), V(t+\Delta), \eta(t+\Delta), \gamma(t+\Delta), \beta(t+\Delta), Z(t+\Delta), Y(t+\Delta))$ for given $(U(t), V(t), \eta(t), \gamma(t), \beta(t), Y(t))$.

Repeated application of the resulting one period scheme will generate a full path:

$$
(U(t), V(t), \eta(t), \gamma(t), \beta(t), Z(t), Y(t)), t \in \mathcal{T},
$$

for the model

$$
\left\{\begin{array}{l}
\mathrm{d} U(t)=\left[\kappa_{\mathrm{U}}\left(\mu_{\mathrm{U}}-U(t)\right)-\lambda_{\mathrm{U}}(t)\right] \mathrm{d} t+\sigma_{\mathrm{U}} \sqrt{U(t)} \mathrm{d} W_{\mathrm{U}}(t)^{\mathbb{Q}}, \\
\mathrm{d} V(t)=\left[\kappa_{\mathrm{V}}\left(\mu_{\mathrm{V}}-V(t)\right)-\lambda_{\mathrm{V}}(t)\right] \mathrm{d} t+\sigma_{\mathrm{V}} \sqrt{V(t)} \mathrm{d} W_{\mathrm{V}}(t)^{\mathbb{Q}}, \\
\mathrm{d} \gamma(t)=\left(c(t, \gamma(t))-\lambda_{\gamma}(t)\right) \mathrm{d} t+d(t, \gamma(t)) \mathrm{d} W_{\gamma}(t)^{\mathbb{Q}}, \gamma(0) \in[-1,1], \\
\mathrm{d} \eta(t)=\left(a(t, \eta(t))-\lambda_{\eta}(t)\right) \mathrm{d} t+b(t, \eta(t)) \mathrm{d} W_{\eta}(t)^{\mathbb{Q}}, \eta(0) \in[-1,1], \\
\mathrm{d} \beta(t)=\left(f(t, \beta(t))-\lambda_{\beta}(t)\right) \mathrm{d} t+g(t, \beta(t)) \mathrm{d} W_{\beta}(t)^{\mathbb{Q}}, \beta(0) \in[-1,1], \\
\mathrm{d} Z(t)=\left(r_{\mathrm{d}}-r_{\mathrm{f}}-\frac{1}{2} U(t)\right) \mathrm{d} t+\sqrt{U(t)} \mathrm{d} W_{\mathrm{X}}(t)^{\mathbb{Q}}, \\
\mathrm{d} Y(t)=\left(r_{\mathrm{f}}-\frac{1}{2} V(t)-\beta(t) \sqrt{V(t)} \sqrt{U(t)}\right) \mathrm{d} t+\sqrt{V(t)} \mathrm{d} W_{\mathrm{S}}(t)^{\mathbb{Q}},
\end{array}\right.
$$

\subsection{Cholesky Decomposition}

As pointed out in [8], a straight discretization of the model in Equation (88) may lead to the problem of leaking correlation. To tackle this problem, we reformulated the system of SDEs (88) with respect to independent Brownian motions as defined below:

${ }^{4}$ As mentioned in Section 2, we apply the log-transform to the underlying asset $S, Y(t)=\ln S(t)$, and to the exchange rate $X, Z(t)=\ln X(t)$. 


$$
\left\{\begin{array}{l}
\mathrm{d} W_{\mathrm{U}}(t)=\mathrm{d} \hat{W}_{\mathrm{U}}(t), \mathrm{d} W_{\mathrm{V}}(t)=\mathrm{d} \hat{W}_{\mathrm{V}}(t), \mathrm{d} W_{\gamma}(t)=\mathrm{d} \hat{W}_{\gamma}(t), \mathrm{d} W_{\eta}(t)=\mathrm{d} \hat{W}_{\eta}(t), \mathrm{d} W_{\beta}(t)=\mathrm{d} \hat{W}_{\beta}(t), \\
\mathrm{d} W_{\mathrm{X}}(t)=\gamma(t) \mathrm{d} \hat{W}_{\mathrm{U}}(t)+\rho_{\mathrm{X} \gamma} \mathrm{d} \hat{W}_{\gamma}(t)+\rho_{\mathrm{X} \beta} \mathrm{d} \hat{W}_{\beta}(t)+\sqrt{1-\gamma(t)^{2}-\rho_{\mathrm{X} \gamma}^{2}-\rho_{\mathrm{X} \beta}^{2}} \mathrm{~d} \hat{W}_{\mathrm{X}}(t), \\
\mathrm{d} W_{\mathrm{S}}(t)=\eta(t) \mathrm{d} \hat{W}_{\mathrm{V}}(t)+\rho_{\mathrm{S} \beta} \mathrm{d} \hat{W}_{\beta}(t)+\tilde{\rho}_{\mathrm{SX}} \mathrm{d} \hat{W}_{\mathrm{X}}(t)+\sqrt{1-\eta(t)^{2}-\rho_{\mathrm{S} \beta}^{2}-\tilde{\rho}_{\mathrm{SX}}^{2}} \mathrm{~d} \hat{W}_{\mathrm{S}}(t),
\end{array}\right.
$$

where

$$
\tilde{\rho}_{\mathrm{SX}} \equiv \frac{\beta(t)-\rho_{\mathrm{X} \beta} \rho_{\mathrm{S} \beta}}{\sqrt{1-\gamma(t)^{2}-\rho_{\mathrm{X} \gamma}^{2}-\rho_{\mathrm{X} \beta}^{2}}} \in[-1,1],
$$

under the conditions that

$$
\gamma(t)^{2}+\rho_{\mathrm{X} \gamma}^{2}+\rho_{\mathrm{X} \beta}^{2}<1, \eta(t)^{2}+\rho_{\mathrm{S} \beta}^{2}+\tilde{\rho}_{\mathrm{SX}}^{2}<1 .
$$

Therefore, the Cholesky decomposition of the correlation matrix $R$ defined in Section 2 can be easily calculated. In the following sections we will discuss the discretization schemes for each of the processes in Equation (88).

\subsection{Discretization Scheme for $U(t)$ and $V(t)$}

To discretize the variance processes $U(t)$ and $V(t)$, we employ the full truncation scheme as in [9], which reads as:

$$
\left\{\begin{array}{l}
\hat{v}(t+\Delta)=v(t)+\mu_{v} \kappa_{v} \Delta-\kappa_{v} \Delta \max (v(t), 0)+\sigma_{v} \sqrt{\max (v(t), 0)} \sqrt{\Delta} Z_{v}, \\
v(t+\Delta)=\max (v(t+\Delta), 0)
\end{array}\right.
$$

which is a valid alternative to the Quadratic-Exponential (QE) scheme proposed in [8].

\subsection{Discretization Scheme for $\eta(t), \gamma(t)$ and $\beta(t)$}

Let us assume first that the correlation processes $\eta(t), \gamma(t)$ and $\beta(t)$ follow an OU process. It is well know that if the process $\rho(t)$ follows an OU dynamics

$$
\mathrm{d} \rho(t)=\kappa_{\rho}\left(\mu_{\rho}-\rho(t)\right) \mathrm{d} t+\sigma_{\rho} \mathrm{d} W_{\rho}(t),
$$

then its exact solution at time $t+\Delta$, given the information available at time $t$ is given by:

$$
\rho(t+\Delta)=\rho(t) \mathrm{e}^{-\kappa_{\rho} \Delta}+\mu_{\rho}\left(1-\mathrm{e}^{-\kappa_{\rho} \Delta}\right)+\sigma_{\rho} \sqrt{\frac{1-\mathrm{e}^{-2 \kappa_{\rho} \Delta}}{2 \kappa_{\rho}}} Z_{\rho},
$$

where $Z_{\rho}$ is a standard Gaussian random variable.

If we instead assume that the correlation processes $\gamma(t), \eta(t)$ and $\gamma(t)$, follow a BJ dynamics, then its SDE is given by:

$$
\mathrm{d} \rho(t)=\kappa_{\rho}\left(\mu_{\rho}-\rho(t)\right) \mathrm{d} t+\sigma_{\rho} \sqrt{1-\rho(t)^{2}} \mathrm{~d} W_{\rho}(t), \text { for } \rho \in\{\gamma, \eta, \beta\} .
$$

Given the lack of an explicit solution for the above SDE, we propose to discretize the dynamics of $\rho(t)$ via either the Euler scheme 


$$
\rho(t+\Delta) \approx \hat{\rho}(t+\Delta)=\kappa_{\rho} \mu_{\rho} \Delta+\left(1-\kappa_{\rho} \Delta\right) \hat{\rho}(t)+\sigma_{\rho} \sqrt{1-\hat{\rho}(t)^{2}} \sqrt{\Delta} Z_{\rho},
$$

or via the Milstein scheme

$$
\begin{aligned}
\rho(t+\Delta) \approx & \hat{\rho}(t+\Delta) \\
= & \kappa_{\rho} \mu_{\rho} \Delta+\left(1-\kappa_{\rho} \Delta\right) \hat{\rho}(t)+\sigma_{\rho} \sqrt{1-\hat{\rho}(t)^{2}} \sqrt{\Delta} Z_{\rho} \\
& -\frac{1}{2} \sigma_{\rho}^{2} \hat{\rho}(t) \Delta\left(Z_{\rho}^{2}-1\right) .
\end{aligned}
$$

\subsection{Discretization Scheme for $Y(t)$ and $Z(t)$}

To discretize the dynamics for the log-underlying price $Y(t)=\ln S(t)$ and for the $\log$-FX rate $Z(t)=\ln X(t)$, we employ the Euler scheme based on the Cholesky decomposition presented in Section 5.1. In particular, we have that

$$
\begin{aligned}
& \hat{Z}(t+\Delta)=\hat{Z}(t)+\left(r_{\mathrm{d}}-r_{\mathrm{f}}-\frac{1}{2} \hat{U}(t)\right) \Delta \\
& +\sqrt{\hat{U}(t)} \sqrt{\Delta}\left(\hat{\gamma}(t) \hat{Z}_{\mathrm{U}}+\rho_{\mathrm{X} \gamma} \hat{Z}_{\gamma}+\rho_{\mathrm{X} \beta} \hat{Z}_{\beta}+\sqrt{1-\hat{\gamma}(t)^{2}-\rho_{\mathrm{X} \gamma}^{2}-\rho_{\mathrm{X} \beta}^{2}} \hat{Z}_{\mathrm{X}}\right),
\end{aligned}
$$

and

$$
\begin{aligned}
& \hat{Y}(t+\Delta)=\hat{Y}(t)+\left(r_{\mathrm{f}}-\frac{1}{2} \hat{V}(t)-\hat{\beta}(t) \sqrt{\hat{U}(t)} \sqrt{\hat{V}(t)}\right) \Delta \\
& +\sqrt{\hat{V}(t)} \sqrt{\Delta}\left(\hat{\eta}(t) \hat{Z}_{\mathrm{V}}+\rho_{\mathrm{S} \beta} \hat{Z}_{\beta}+\tilde{\rho}_{\mathrm{SX}} \hat{Z}_{\mathrm{X}}+\sqrt{1-\hat{\eta}(t)^{2}-\rho_{\mathrm{S} \beta}^{2}-\tilde{\rho}_{\mathrm{SX}}^{2}} \hat{Z}_{\mathrm{S}}\right) .
\end{aligned}
$$

We conclude this section by remarking that, although the FX rate does not explicitly enter in the payoff of a quanto option, we do still need to simulate it, as the standard Gaussian random variable $\hat{Z}_{\mathrm{x}}$ enters explicitly in the dynamics for the log-underlying price $Y$, as shown in Equation (97).

\section{Numerical Experiments}

In this section we compare the option prices using the closed-form approximation Formula (18) to the prices computed by performing a Monte Carlo simulation according to the numerical scheme presented in Section 5. In particular, we have considered quanto call options on a foreign stock priced in the domestic currency, where the majority of the model parameters are defined at the bottom of Table 1.

In Table 1 we have reported the results using different stochastic processes specification (namely the Ornstein-Uhlenbeck and the bounded Jacobi processes) and for different strikes. Six different scenarios have been considered under the following assumptions (as said all the other model parameters are at the bottom of the table and they have been kept fixed along the scenarios):

1) $\beta(0)=\mu_{\beta}=0$ and $\rho_{\mathrm{S} \beta}=\rho_{\mathrm{X} \beta}=\rho_{\mathrm{S} \eta}=\rho_{\mathrm{X} \gamma}=0$,

2) $\beta(0)=0, \mu_{\beta}=0.5$ and $\rho_{\mathrm{S} \beta}=\rho_{\mathrm{X} \beta}=\rho_{\mathrm{S} \eta}=\rho_{\mathrm{X} \gamma}=0$,

3) $\beta(0)=0, \mu_{\beta}=-0.5$ and $\rho_{\mathrm{S} \beta}=\rho_{\mathrm{X} \beta}=\rho_{\mathrm{S} \eta}=\rho_{\mathrm{X} \gamma}=0$,

4) $\beta(0)=\mu_{\beta}=0, \rho_{\mathrm{S} \beta}=\rho_{\mathrm{X} \beta}=0.5$ and $\rho_{\mathrm{S} \eta}=\rho_{\mathrm{X} \gamma}=0$, 
Table 1. The other parameters are assumed as: $S(0)=100, X(0)=1, r_{\mathrm{d}}=0.03, r_{\mathrm{f}}=0.05, T=1, U(0)=V(0)=0.02$, $\mu_{\mathrm{U}}=\mu_{\mathrm{V}}=0.03, \kappa_{\mathrm{U}}=\kappa_{\mathrm{V}}=2.1, \sigma_{\mathrm{U}}=\sigma_{\mathrm{V}}=0.1$, and $\eta(0)=\gamma(0)=-0.2, \mu_{\eta}=\mu_{\gamma}=-0.3, \kappa_{\eta}=\kappa_{\gamma}=3.4, \sigma_{\eta}=\sigma_{\gamma}=0.1$. The numbers in round brackets represent the standard deviations. The number of Monte Carlo simulations is equal to $100,000$.

\begin{tabular}{|c|c|c|c|c|c|c|c|c|c|}
\hline \multirow{2}{*}{ Scenario } & \multicolumn{3}{|c|}{ Model Parameters } & \multicolumn{3}{|c|}{ OU Stochastic Correlation } & \multicolumn{3}{|c|}{ BJ Stochastic Correlation } \\
\hline & $\beta(0)$ & $\mu_{\beta}$ & Strike & MC Price & Approx & Rel Error & MC Price & Approx & Rel Error \\
\hline \multirow[t]{9}{*}{1} & 0 & 0.0 & 80 & $24.6479(0.0506)$ & 24.6472 & $-0.00 \%$ & $24.6483(0.0506)$ & 24.6476 & $-0.00 \%$ \\
\hline & & & 85 & $20.1757(0.0489)$ & 20.1426 & $-0.16 \%$ & $20.1761(0.0489)$ & 20.1430 & $-0.16 \%$ \\
\hline & & & 90 & $16.0172(0.0463)$ & 15.8726 & $-0.91 \%$ & $16.0177(0.0463)$ & 15.8731 & $-0.91 \%$ \\
\hline & & & 95 & $12.2973(0.0423)$ & 12.0183 & $-2.32 \%$ & $12.2976(0.0427)$ & 12.0186 & $-2.32 \%$ \\
\hline & & & 100 & $9.1217(0.0384)$ & 8.7531 & $-4.21 \%$ & $9.1221(0.0384)$ & 8.7535 & $-4.21 \%$ \\
\hline & & & 105 & $6.5438(0.0335)$ & 6.1505 & $-6.40 \%$ & $6.5440(0.0335)$ & 6.1507 & $-6.39 \%$ \\
\hline & & & 110 & $4.5422(0.0285)$ & 4.1780 & $-8.72 \%$ & $4.5425(0.0285)$ & 4.1784 & $-8.71 \%$ \\
\hline & & & 115 & $3.0571(0.0236)$ & 2.7525 & $-11.07 \%$ & $3.0573(0.0236)$ & 2.7527 & $-11.07 \%$ \\
\hline & & & 120 & $2.0019(0.0192)$ & 1.7642 & $-13.48 \%$ & $2.0020(0.0192)$ & 1.7643 & $-13.47 \%$ \\
\hline \multirow[t]{9}{*}{2} & 0 & 0.5 & 80 & $23.8150(0.0498)$ & 23.8102 & $-0.02 \%$ & $23.8138(0.0498)$ & 23.8090 & $-0.02 \%$ \\
\hline & & & 85 & $19.3741(0.0480)$ & 19.3008 & $-0.38 \%$ & $19.3729(0.0480)$ & 19.2995 & $-0.38 \%$ \\
\hline & & & 90 & $15.2703(0.0453)$ & 15.0387 & $-1.54 \%$ & $15.2693(0.0453)$ & 15.0376 & $-1.54 \%$ \\
\hline & & & 95 & $11.6337(0.0416)$ & 11.2522 & $-3.39 \%$ & $11.6329(0.0416)$ & 11.2513 & $-3.39 \%$ \\
\hline & & & 100 & $8.5556(0.0371)$ & 8.0958 & $-5.68 \%$ & $8.5549(0.0371)$ & 8.0949 & $-5.68 \%$ \\
\hline & & & 105 & $6.0753(0.0321)$ & 5.6123 & $-8.25 \%$ & $6.0748(0.0321)$ & 5.6117 & $-8.25 \%$ \\
\hline & & & 110 & $4.1717(0.0271)$ & 3.7607 & $-10.93 \%$ & $4.1713(0.0271)$ & 3.7602 & $-10.93 \%$ \\
\hline & & & 115 & $2.7783(0.0223)$ & 2.4455 & $-13.61 \%$ & $2.7781(0.0223)$ & 2.4452 & $-13.61 \%$ \\
\hline & & & 120 & $1.7961(0.0180)$ & 1.5425 & $-16.44 \%$ & $1.7958(0.0180)$ & 1.5422 & $-16.44 \%$ \\
\hline \multirow[t]{10}{*}{3} & 0 & -0.5 & 80 & $25.4883(0.0514)$ & 25.4847 & $-0.01 \%$ & $25.4900(0.0514)$ & 25.4864 & $-0.01 \%$ \\
\hline & & & 85 & $20.9794(0.0498)$ & 20.9712 & $-0.04 \%$ & $20.9811(0.0498)$ & 20.9728 & $-0.04 \%$ \\
\hline & & & 90 & $16.773(0.0473)$ & 16.7199 & $-0.32 \%$ & $16.7746(0.0473)$ & 16.7217 & $-0.32 \%$ \\
\hline & & & 95 & $12.9872(0.0439)$ & 12.8213 & $-1.29 \%$ & $12.9885(0.0439)$ & 12.8226 & $-1.29 \%$ \\
\hline & & & 100 & $9.7213(0.0396)$ & 9.4572 & $-2.79 \%$ & $9.7224(0.0396)$ & 9.4585 & $-2.79 \%$ \\
\hline & & & 105 & $7.0384(0.0348)$ & 6.7269 & $-4.63 \%$ & $7.0394(0.0348)$ & 6.7281 & $-4.63 \%$ \\
\hline & & & 110 & $4.9338(0.0298)$ & 4.6267 & $-6.64 \%$ & $4.9345(0.0298)$ & 4.6275 & $-6.63 \%$ \\
\hline & & & 115 & $3.3552(0.0249)$ & 3.0870 & $-8.69 \%$ & $3.3557(0.0249)$ & 3.0876 & $-8.68 \%$ \\
\hline & & & 120 & $2.2245(0.0204)$ & 2.0091 & $-10.72 \%$ & $2.2248(0.0204)$ & 2.0094 & $-10.72 \%$ \\
\hline & $\rho_{\mathrm{s} \beta}$ & $\rho_{\mathrm{x} \beta}$ & Strike & MC Price & Approx & Rel Error & MC Price & Approx & Rel Error \\
\hline \multirow[t]{5}{*}{4} & 0.5 & 0.5 & 80 & $24.6494(0.0506)$ & 24.6487 & $-0.00 \%$ & $24.6492(0.0506)$ & 24.6485 & $-0.00 \%$ \\
\hline & & & 85 & $20.1723(0.0489)$ & 20.1386 & $-0.02 \%$ & $20.1718(0.0489)$ & 20.1380 & $-0.18 \%$ \\
\hline & & & 90 & $16.0182(0.0463)$ & 15.8726 & $-0.92 \%$ & $16.0172(0.0463)$ & 15.8712 & $-0.92 \%$ \\
\hline & & & 95 & $12.3098(0.0427)$ & 12.0310 & $-2.32 \%$ & $12.3086(0.0427)$ & 12.0292 & $-2.32 \%$ \\
\hline & & & 100 & $9.1403(0.0383)$ & 8.7727 & $-4.19 \%$ & $9.1389(0.0383)$ & 8.7705 & $-4.20 \%$ \\
\hline
\end{tabular}




\begin{tabular}{|c|c|c|c|c|c|c|c|c|c|}
\hline & & & 105 & $6.5570(0.0334)$ & 6.1636 & $-6.38 \%$ & $6.5555(0.0334)$ & 6.1614 & $-6.40 \%$ \\
\hline & & & 110 & $4.5517(0.0284)$ & 4.1869 & $-8.71 \%$ & $4.5502(0.0284)$ & 4.1847 & $-8.73 \%$ \\
\hline & & & 115 & $3.0590(0.0235)$ & 2.7530 & $-11.12 \%$ & $3.0576(0.0235)$ & 2.7510 & $-11.14 \%$ \\
\hline & & & 120 & $1.9949(0.0191)$ & 1.7552 & $-13.65 \%$ & $1.9935(0.0191)$ & 1.7534 & $-13.69 \%$ \\
\hline \multirow[t]{10}{*}{5} & -0.5 & -0.5 & 80 & $24.6577(0.0508)$ & 24.6571 & $-0.00 \%$ & $24.6594(0.0508)$ & 24.6588 & $-0.00 \%$ \\
\hline & & & 85 & $20.1758(0.0492)$ & 20.1431 & $-0.16 \%$ & $20.1776(0.0492)$ & 20.1451 & $-0.16 \%$ \\
\hline & & & 90 & $16.0215(0.0465)$ & 15.8785 & $-0.90 \%$ & $16.0236(0.0466)$ & 15.8811 & $-0.90 \%$ \\
\hline & & & 95 & $12.3172(0.0430)$ & 12.0432 & $-2.27 \%$ & $12.3196(0.0430)$ & 12.0464 & $-2.27 \%$ \\
\hline & & & 100 & $9.1541(0.0386)$ & 8.7932 & $-4.10 \%$ & $9.1568(0.0386)$ & 8.7969 & $-4.09 \%$ \\
\hline & & & 105 & $6.5792(0.0337)$ & 6.1939 & $-6.22 \%$ & $6.5818(0.0337)$ & 6.1974 & $-6.20 \%$ \\
\hline & & & 110 & $4.5789(0.0286)$ & 4.2220 & $-8.45 \%$ & $4.5811(0.0286)$ & 4.2249 & $-8.43 \%$ \\
\hline & & & 115 & $3.0899(0.0238)$ & 2.7908 & $-10.72 \%$ & $3.0919(0.0238)$ & 2.7934 & $-10.69 \%$ \\
\hline & & & 120 & $2.0307(0.0194)$ & 1.7970 & $-13.01 \%$ & $2.0324(0.0194)$ & 1.7991 & $-12.96 \%$ \\
\hline & $\rho_{\mathrm{S} \eta}$ & $\rho_{\mathrm{x} \gamma}$ & Strike & MC Price & Approx & Rel Error & MC Price & Approx & Rel Error \\
\hline \multirow[t]{9}{*}{6} & -0.5 & -0.5 & 80 & $24.6515(0.0506)$ & 24.6508 & $-0.00 \%$ & $24.6515(0.0506)$ & 24.6508 & $-0.00 \%$ \\
\hline & & & 85 & $20.1751(0.0490)$ & 20.1416 & $-0.16 \%$ & $20.1748(0.0490)$ & 20.1412 & $-0.18 \%$ \\
\hline & & & 90 & $16.0203(0.0463)$ & 15.8749 & $-0.92 \%$ & $16.0195(0.0463)$ & 15.8738 & $-0.93 \%$ \\
\hline & & & 95 & $12.3113(0.0427)$ & 12.0326 & $-2.32 \%$ & $12.3101(0.0427)$ & 12.0308 & $-2.32 \%$ \\
\hline & & & 100 & $9.1420(0.0383)$ & 8.7744 & $-4.19 \%$ & $9.1406(0.0383)$ & 8.7723 & $-4.20 \%$ \\
\hline & & & 105 & $6.5574(0.0335)$ & 6.1638 & $-6.39 \%$ & $6.5562(0.0334)$ & 6.1619 & $-6.40 \%$ \\
\hline & & & 110 & $4.5516(0.0284)$ & 4.1866 & $-8.72 \%$ & $4.5562(0.0284)$ & 4.1900 & $-8.74 \%$ \\
\hline & & & 115 & $3.0605(0.0236)$ & 2.7545 & $-11.11 \%$ & $3.0592(0.0236)$ & 2.7527 & $-11.14 \%$ \\
\hline & & & 120 & $1.9973(0.0192)$ & 1.7578 & $-13.62 \%$ & $1.9959(0.0192)$ & 1.7560 & $-13.66 \%$ \\
\hline
\end{tabular}

5) $\beta(0)=\mu_{\beta}=0, \quad \rho_{\mathrm{S} \beta}=\rho_{\mathrm{X} \beta}=-0.5$ and $\rho_{\mathrm{S} \eta}=\rho_{\mathrm{X} \gamma}=0$,

6) $\beta(0)=\mu_{\beta}=0, \quad \rho_{\mathrm{S} \beta}=\rho_{\mathrm{X} \beta}=0.5$ and $\rho_{\mathrm{S} \eta}=\rho_{\mathrm{X} \gamma}=-0.5$.

As pointed out in [2] and in [1], one should choose a large value for the mean reversion rate of the correlation processes and a small value for the volatility coefficient in order to ensure that the generated correlations by the OU process lie in the interval $(-1,1)$, while for the BJ process the condition in Equation (58) has to be fulfilled. Besides that, care has to be taken also in the choice of the other model parameters for the stochastic processes (namely the initial value and the long term mean level) because of the conditions implied by the Cholesky decomposition, see Equation (91). We remark that in all the scenarios considered in this Section all the model constraints have been taken into account.

If we look at the relative errors reported in Table 1, we can see that the ap- 
proximations in both models give accurate results, but the accuracy of the approximation decreases when passing from in-the-money to out-of-the-money options. If we consider Scenarios 1 - 3 first, we can see the effect of changing the long-term mean level for the correlation process $\beta(t)$ on the option value. Indeed, the fact that the price in Scenario 2 (resp. 3) is lower (higher) than the one in Scenario 1 can be explained from the fact that the drift of the underlying process decreases (increases), under the same volatility assumption. If we compare Scenarios 4 and 5 with Scenario 1, we can see that increasing (decreasing) the correlations between the stochastic drivers for the underlying price (resp. FX rate) and the correlation process $\beta$ does not have a huge impact on the option price. This has been already noted in [2] and it is due to the fact that we are pricing the option under a low volatility regime for the correlation process $\beta$. Also, this explains as well why the option price given by the two model specifications for the correlation process are very close. The same conclusion holds for Scenario 6 where we have assumed a non-zero correlation between the stochastic drivers for the underlying price (resp. FX rate) and the correlation process $\eta$ (resp. $\gamma$ ).

\section{Conclusion and Future Work}

In this paper we have incorporated a stochastic correlation structure into the pricing of FX quanto options where both the dynamics for the underlying asset and for the exchange rate are given by a stochastic volatility model. This has been done not only assuming a stochastic correlation between the underlying asset and its variance process (and the same between the exchange rate and its variance process), but also assuming a stochastic correlation between the underlying asset and the exchange rate. In particular, under the assumption that the set of stochastic correlation processes follow an Ornstein-Uhlenbeck and a bounded Jacobi process respectively, we have derived a closed-form approximation for the characteristic function of the underlying asset, by approximating non-affine terms in the model dynamics. The comparison of both approximations with the Monte Carlo method has also been discussed. Given the fact that the analytical tractability of the formulas allows for fast pricing and calibration purposes, improvements of the present work could be to focus on the calibration of the model parameters by looking at real market quotes from the FX market and also to run more scenario analysis under stressed market conditions. We leave them as future work.

\section{Acknowledgements}

We thank the Editor and two referees for their comments.

\section{Conflicts of Interest}

The author declares no conflicts of interest regarding the publication of this paper. 


\section{References}

[1] Teng, L., Ehrhardt, M. and Günther, M. (2018) Quanto Pricing in Stochastic Correlation Models. International Journal of Theoretical and Applied Finance, 21, Article ID: 1850038. https://doi.org/10.1142/S0219024918500383

[2] Teng, L., Ehrhardt, M. and Günther, M. (2016) On the Heston Model with Stochastic Correlation. International Journal of Theoretical and Applied Finance, 19, Article ID: 1650033. https://doi.org/10.1142/S0219024916500333

[3] Heston, S.L. (1993) A Closed-Form Solution for Options with Stochastic Volatility with Applications to Bond and Currency Options. The Review of Financial Studies, 6, 327-343.

[4] Cox, J.C., Ingersoll, J.E. and Ross, S.A. (1985) A Theory of the Term Structure of Interest Rates. Econometrica, 53, 385-407. https://doi.org/10.2307/1911242

[5] Grzelak, L.A. and Oosterlee, C.W. (2011) On the Heston Model with Stochastic Interest Rates. SIAM Journal on Financial Mathematics, 2, 255-286. https://doi.org/10.1137/090756119

[6] Van Emmerich, C. (2006) Modelling Correlation as a Stochastic Process. University of Wuppertal, Wuppertal.

[7] Van Emmerich, C. (2007) A Square Root Process for Modelling Correlation. University of Wuppertal, Wuppertal.

[8] Andersen, L. (2008) Simple and Efficient Simulation of the Heston Stochastic Volatility Model. Journal of Computational Finance, 11, 1-42. https://doi.org/10.21314/JCF.2008.189

[9] Lord, R., Koekkoek, R. and van Dijk, D. (2010) A Comparison of Biased Simulation Schemes for Stochastic Volatility Models. Quantitative Finance, 10, 177-194. https://doi.org/10.1080/14697680802392496 


\section{Appendix}

\section{A.1. The Proof of Proposition 1}

In order to find a first order approximation for $\mathbb{E}[\sqrt{v(t)}]$ we can apply the so-called delta method which states that a function $\phi(X)$ can be approximated by a first order Taylor expansion around $\mathbb{E}[X]$, for a given random variable $X$, with expectation $\mathbb{E}[X]$ and variance $\operatorname{Var}[X]$, under the assumption that the first two moments of $X$ exist and that the first derivative of $\phi(X)$ with respect to $X$ exists and is sufficiently smooth.

Therefore, we obtain by first order Taylor expansion:

$$
\phi(X) \approx \phi(\mathbb{E}[X])+(X-\mathbb{E}[X]) \frac{\partial}{\partial X} \phi(\mathbb{E}[X]) .
$$

Now, the variance of $\phi(X)$ can be approximated by taking the variance of the right-hand side of Equation (98), which reads as follows:

$$
\begin{aligned}
\operatorname{Var}[\phi(X)] & \approx \operatorname{Var}\left[\phi(\mathbb{E}[X])+(X-\mathbb{E}[X]) \frac{\partial}{\partial X} \phi(\mathbb{E}[X])\right] \\
& =\left(\frac{\partial}{\partial X} \phi(\mathbb{E}[X])\right)^{2} \operatorname{Var}[X] .
\end{aligned}
$$

Now, if we apply this result to the function $\phi(v(t))=\sqrt{v(t)}$, we have that

$$
\operatorname{Var}[\sqrt{v(t)}] \approx\left(\frac{1}{2} \frac{1}{\sqrt{\mathbb{E}[v(t)]}}\right)^{2} \operatorname{Var}[v(t)]=\frac{1}{4} \frac{\operatorname{Var}[v(t)]}{\mathbb{E}[v(t)]} .
$$

Besides it, we know from the definition of variance that

$$
\operatorname{Var}[\sqrt{v(t)}]=\mathbb{E}[v(t)]-(\mathbb{E}[v(t)])^{2},
$$

and combining Equaitons (100) and (101), we obtain the following approximation for $\mathbb{E}[\sqrt{v(t)}]$ :

$$
\mathbb{E}[\sqrt{v(t)}] \approx \sqrt{\mathbb{E}[v(t)]-\frac{1}{4} \frac{\operatorname{Var}[v(t)]}{\mathbb{E}[v(t)]}} .
$$

Since $v(t)$ is a square root process, then its explicit solution at time $t$ is given by:

$$
v(t)=v(0) \mathrm{e}^{-\kappa_{v} t}+\mu_{v}\left(1-\mathrm{e}^{-\kappa_{v} t}\right)+\sigma_{v} \int_{0}^{t} \mathrm{e}^{\kappa_{v}(s-t)} \sqrt{v(s)} \mathrm{d} W_{v}(s) .
$$

Further calculations show that the expectation and the variance of $v(t)$ can be expressed as follows:

$$
\mathbb{E}[v(t)]=\tilde{a}_{v}(t)\left(\tilde{b}_{v}+\tilde{c}_{v}(t)\right), \operatorname{Var}[v(t)]=\tilde{a}_{v}(t)^{2}\left(2 \tilde{b}_{v}+4 \tilde{c}_{v}(t)\right),
$$

where the coefficients $\tilde{a}_{v}(t), b_{v}, c_{v}(t)$ are given by:

$$
\tilde{a}_{v}(t)=\frac{\sigma_{v}^{2}}{4 \kappa_{v}}\left(1-\mathrm{e}^{-\kappa_{v} t}\right), \tilde{b}_{v}=\frac{4 \kappa_{v} \mu_{v}}{\sigma_{v}^{2}}, \tilde{c}_{v}(t)=\frac{4 \kappa_{v} v(0) \mathrm{e}^{-\kappa_{v} t}}{\sigma_{v}^{2}\left(1-\mathrm{e}^{-\kappa_{v} t}\right)} .
$$

Now, substituting these expressions in Equation (102) gives 


$$
\begin{aligned}
\mathbb{E}[\sqrt{v(t)}] & \approx \sqrt{\tilde{a}_{v}(t)\left(\tilde{b}_{v}+\tilde{c}_{v}(t)\right)-\frac{1}{4} \frac{\tilde{a}_{v}(t)^{2}\left(2 \tilde{b}_{v}+4 \tilde{c}_{v}(t)\right)}{\tilde{a}_{v}(t)\left(\tilde{b}_{v}+\tilde{c}_{v}(t)\right)}} \\
& =\sqrt{\tilde{a}_{v}(t)\left(\tilde{c}_{v}(t)-1\right)+\tilde{a}_{v}(t) \tilde{b}_{v}+\frac{\tilde{a}_{v}(t) \tilde{b}_{v}}{2\left(\tilde{b}_{v}+\tilde{c}_{v}(t)\right)}} .
\end{aligned}
$$

Finally, if we denote by $\Lambda(t)$ the above expectation, then $\Lambda(t)$ can be further approximated by a function of the following form:

$$
\Lambda(t) \approx \tilde{\Lambda}(t)=m_{v}+n_{v} \mathrm{e}^{-l_{v} t},
$$

where the coefficients $m_{v}, n_{v}, l_{v}$ are found by matching the functions $\Lambda(t)$ and $\tilde{\Lambda}(t)$ for $t \rightarrow+\infty, t \rightarrow 0$ and for $t \rightarrow 1$.

In particular, it can be proved that (see Result 3.3 in [5])

$$
\left\{\begin{array}{l}
\lim _{t \rightarrow+\infty} \Lambda(t)=\sqrt{\mu_{v}-\frac{\sigma_{v}^{2}}{8 \kappa_{v}}}=m_{v}=\lim _{t \rightarrow+\infty} \tilde{\Lambda}(t), \\
\lim _{t \rightarrow 0} \Lambda(t)=\sqrt{v(0)}=m_{v}+n_{v}=\lim _{t \rightarrow 0} \tilde{\Lambda}(t), \\
\lim _{t \rightarrow 1} \Lambda(t)=\Lambda(1)=m_{v}+n_{v} \mathrm{e}^{-l_{v}}=\lim _{t \rightarrow 1} \tilde{\Lambda}(t),
\end{array}\right.
$$

from which it follows that

$$
m_{v}=\sqrt{\mu_{v}-\frac{\sigma_{v}^{2}}{8 \kappa_{v}}}, n_{v}=\sqrt{\nu_{0}}-m_{v}, l_{v}=-\ln \left(\frac{\hat{d}_{v}-m_{v}}{n_{v}}\right),
$$

where we have defined

$$
\hat{d}_{v}=\sqrt{\left(v_{0} \mathrm{e}^{-\kappa_{v}}-\frac{\sigma_{v}^{2}\left(1-\mathrm{e}^{-\kappa_{v}}\right)}{4 \kappa_{v}}\right)+\mu_{v}\left(1-\mathrm{e}^{-\kappa_{v}}\right)+\frac{\sigma_{v}^{2} \mu_{v}\left(1-\mathrm{e}^{-\kappa_{v}}\right)^{2}}{8 \kappa_{v} \mu_{v}+8 \kappa_{v} \mathrm{e}^{-\kappa_{v}}\left(v_{0}-\mu_{v}\right)} .}
$$

This completes the proof.

\section{A.2. The Proof of Lemma 1}

Since the system generating the pricing PDE for $P_{1}$ (30) is of affine form, we look for a solution $\phi_{1}=\phi_{1}(\tau, \omega)$ of the following form:

$$
\begin{aligned}
\phi_{1}^{\mathrm{OU}}= & \exp \left(A_{1}(\tau, \omega)+B_{1}(\tau, \omega) v(t)+C_{1}(\tau, \omega) u(t)+D_{1}(\tau, \omega) z(t)\right. \\
& \left.+E_{1}(\tau, \omega) \beta(t)+F_{1}(\tau, \omega) \eta(t)+G_{1}(\tau, \omega) \gamma(t)+i \omega y(t)\right),
\end{aligned}
$$

with

$$
A_{1}(0, \omega)=B_{1}(0, \omega)=C_{1}(0, \omega)=D_{1}(0, \omega)=E_{1}(0, \omega)=F_{1}(0, \omega)=G_{1}(0, \omega)=0 .
$$

Substituting all the partial derivatives of $\phi_{1}$ into (30) gives:

$$
\begin{aligned}
& -\left(\frac{\partial A_{1}}{\partial \tau}+v \frac{\partial B_{1}}{\partial \tau}+u \frac{\partial C_{1}}{\partial \tau}+z \frac{\partial D_{1}}{\partial \tau}+\beta \frac{\partial E_{1}}{\partial \tau}+\eta \frac{\partial F_{1}}{\partial \tau}+\gamma \frac{\partial G_{1}}{\partial \tau}\right) \\
& +\left(r_{\mathrm{f}}+\frac{1}{2} v-\beta \mathbb{E}[\sqrt{v}] \mathbb{E}[\sqrt{u}]\right) i \omega+\left(r_{\mathrm{d}}-r_{\mathrm{f}}-\frac{1}{2} u+\beta \mathbb{E}[\sqrt{v}] \mathbb{E}[\sqrt{u}]\right) D_{1} \\
& +\left(\kappa_{\eta}\left(\mu_{\eta}-\eta\right)+\rho_{\mathrm{S} \eta} \sigma_{\eta} \mathbb{E}[\sqrt{v}]\right) F_{1}+\left(\kappa_{\mathrm{v}}\left(\mu_{\mathrm{v}}-v\right)+\sigma_{\mathrm{v}} \mathbb{E}[v] \eta\right) B_{1} \\
& +\kappa_{\mathrm{U}}\left(\mu_{\mathrm{U}}-u\right) C_{1}+\left(\kappa_{\beta}\left(\mu_{\beta}-\beta\right)+\rho_{\mathrm{S} \beta} \sigma_{\beta} \mathbb{E}[\sqrt{v}]\right) E_{1}+\kappa_{\gamma}\left(\mu_{\gamma}-\gamma\right) G_{1}
\end{aligned}
$$




$$
\begin{aligned}
& -\frac{1}{2} \omega^{2} v+\frac{1}{2} u D_{1}^{2}+\frac{1}{2} \sigma_{\mathrm{v}}^{2} v B_{1}^{2}+\frac{1}{2} \sigma_{\mathrm{U}}^{2} u C_{1}^{2}+\frac{1}{2} \sigma_{\beta}^{2} E_{1}^{2}+\frac{1}{2} \sigma_{\eta}^{2} F_{1}^{2}+\frac{1}{2} \sigma_{\gamma}^{2} G_{1}^{2} \\
& +\beta \mathbb{E}[\sqrt{v}][\sqrt{u}] i \omega D_{1}+\sigma_{\mathrm{v}} \mathbb{E}[v] \eta i \omega B_{1}+\sigma_{\mathrm{U}} \mathbb{E}[u] \gamma D_{1} C_{1} \\
& +\rho_{\mathrm{S} \beta} \sigma_{\beta} \mathbb{E}[\sqrt{v}] i \omega E_{1}+\rho_{\mathrm{S} \eta} \sigma_{\eta} \mathbb{E}[\sqrt{v}] i \omega F_{1}+\rho_{X \beta} \sigma_{\beta} \mathbb{E}[\sqrt{u}] D_{1} E_{1} \\
& +\rho_{\mathrm{X} \gamma} \sigma_{\gamma} \mathbb{E}[\sqrt{u}] D_{1} G_{1}=0 .
\end{aligned}
$$

Now, collecting together homogenous terms in $y, z, v, u, \beta, \eta, \gamma$ gives the following system of equations:

$$
\begin{gathered}
\frac{\partial B_{1}}{\partial \tau}+\kappa_{\mathrm{V}} B_{1}-\frac{1}{2} \sigma_{\mathrm{v}}^{2} B_{1}^{2}+\frac{1}{2} \omega(\omega-i)=0, \\
\frac{\partial C_{1}}{\partial \tau}+\kappa_{\mathrm{U}} C_{1}-\frac{1}{2} \sigma_{\mathrm{U}}^{2} C_{1}^{2}-\frac{1}{2} D_{1}\left(D_{1}-1\right)=0, \\
\frac{\partial D_{1}}{\partial \tau}=0, \\
\frac{\partial E_{1}}{\partial \tau}+\kappa_{\beta} E_{1}-\mathbb{E}[\sqrt{u}] \mathbb{E}[\sqrt{v}]\left(i \omega\left(D_{1}-1\right)+D_{1}\right)=0, \\
\frac{\partial F_{1}}{\partial \tau}+\kappa_{\eta} F_{1}-\sigma_{\mathrm{v}} \mathbb{E}[v] B_{1}(i \omega+1)=0, \\
\frac{\partial G_{1}}{\partial \tau}+\kappa_{\gamma} G_{1}-\sigma_{\mathrm{U}} \mathbb{E}[u] D_{1} C_{1}=0,
\end{gathered}
$$

and

$$
\begin{aligned}
\frac{\partial A_{1}}{\partial \tau}= & r_{\mathrm{f}} i \omega+\left(\kappa_{\eta} \mu_{\eta}+\rho_{\mathrm{S} \eta} \sigma_{\eta} \mathbb{E}[\sqrt{v}](i \omega+1)\right) F_{1}+\kappa_{\mathrm{V}} \mu_{\mathrm{V}} B_{1}+\kappa_{\mathrm{U}} \mu_{\mathrm{U}} C_{1} \\
& +\left(\kappa_{\beta} \mu_{\beta}+\rho_{\mathrm{S} \beta} \sigma_{\beta} \mathbb{E}[\sqrt{v}](i \omega+1)\right) E_{1}+\kappa_{\gamma} \mu_{\gamma} G_{1}+\frac{1}{2} \sigma_{\beta}^{2} E_{1}^{2}+\frac{1}{2} \sigma_{\eta}^{2} F_{1}^{2} \\
& +\frac{1}{2} \sigma_{\gamma}^{2} G_{1}^{2}+\rho_{X \beta} \sigma_{\beta} \mathbb{E}[\sqrt{u}] D_{1} E_{1}+\rho_{\mathrm{X} \gamma} \sigma_{\gamma} \mathbb{E}[\sqrt{u}] D_{1} G_{1}+\left(r_{\mathrm{d}}-r_{\mathrm{f}}\right) D_{1} .
\end{aligned}
$$

From Equation (115), due to the initial condition $D_{1}(0, \omega)=0$, we obtain $D_{1}(\tau, \omega) \equiv 0$, which is consistent with the fact that the option payoff does not depend explicitly on the exchange rate $Z(t)=\ln X(t)$.

The fact that $D_{1}(\tau, \omega)=0$ implies that also $G_{1}(\tau, \omega)=0$, because of the initial condition $G_{1}(0, \omega)=0$.

Calculation of $B_{1}(\tau, \omega)$

Equation (113) is a Riccati equation, whose corresponding second order ordinary differential equation (ODE) reads as follows:

$$
\theta^{\prime \prime}+\kappa_{\mathrm{V}} \theta^{\prime}-\frac{1}{4} \sigma_{\mathrm{V}}^{2} \omega(\omega-i)=0 .
$$

The solution of the above equation is:

$$
\theta_{1,2}=\frac{-\kappa_{\mathrm{v}} \pm \sqrt{\kappa_{\mathrm{V}}^{2}+\sigma_{\mathrm{v}}^{2} \omega(\omega-i)}}{2},
$$

and therefore, the solution of the Riccati Equation (113) is given by: 


$$
B_{1}(\tau, \omega)=-\frac{2}{\sigma_{\mathrm{V}}^{2}}\left(\frac{\theta_{3} \theta_{1} \mathrm{e}^{\theta_{1} \tau}+\theta_{2} \mathrm{e}^{\theta_{2} \tau}}{\theta_{3} \mathrm{e}^{\theta_{1} \tau}+\mathrm{e}^{\theta_{2} \tau}}\right) .
$$

Using the initial condition $B(0, \omega)=0$, we find that the constant is equal to $\theta_{3}=-\frac{\theta_{2}}{\theta_{1}}$.

Further calculations show that $B_{1}(\tau, \omega)$ can be expressed as follows:

$$
B_{1}(\tau, \omega)=-\frac{2 \theta_{2}}{\sigma_{\mathrm{V}}^{2}} \frac{1-\mathrm{e}^{\left(\theta_{1}-\theta_{2}\right) \tau}}{1-\frac{\theta_{2}}{\theta_{1}} \mathrm{e}^{\left(\theta_{1}-\theta_{2}\right) \tau}} .
$$

Now, it can be shown that

$$
\begin{gathered}
\frac{\theta_{2}}{\theta_{1}}=\bar{B}_{2}=\frac{-\kappa_{\mathrm{V}}+\sqrt{\kappa_{\mathrm{V}}^{2}+\sigma_{\mathrm{V}}^{2} \omega(\omega-i)}}{\kappa_{\mathrm{V}}+\sqrt{\kappa_{\mathrm{V}}^{2}+\sigma_{\mathrm{V}}^{2} \omega(\omega-i)}}=\frac{-\kappa_{\mathrm{V}}+\bar{B}_{1}}{\kappa_{\mathrm{V}}+\bar{B}_{1}}, \\
\theta_{1}-\theta_{2}=-\bar{B}_{1},-\frac{2 \theta_{2}}{\sigma_{\mathrm{V}}^{2}}=\frac{\kappa_{\mathrm{V}}-\bar{B}_{1}}{\sigma_{\mathrm{V}}^{2}}, \text { with } \bar{B}_{1}=\sqrt{\kappa_{\mathrm{V}}^{2}+\sigma_{\mathrm{V}}^{2} \omega(\omega-i)},
\end{gathered}
$$

and hence $B_{1}(\tau, \omega)$ reads as:

$$
B_{1}(\tau, \omega)=\frac{\kappa_{\mathrm{V}}-\bar{B}_{1}}{\sigma_{\mathrm{V}}^{2}} \frac{1-\mathrm{e}^{-\bar{B}_{1} \tau}}{1-\bar{B}_{2} \mathrm{e}^{-\bar{B}_{1} \tau}} .
$$

Calculation of $C_{1}(\tau, \omega)$

If we focus now on Equation (114), this is again a Riccati-type equation in $C_{1}$ and its corresponding second order ODE reads as:

$$
\theta^{\prime \prime}+\kappa_{\mathrm{U}} \theta^{\prime}=0,
$$

and $\theta_{1}=-\kappa_{\mathrm{U}}, \theta_{2}=0$.

A general solution for $C_{1}$ can therefore be written as follows:

$$
C_{1}(\tau, \omega)=-\frac{2}{\sigma_{U}^{2}} \frac{\theta_{3} \theta_{1} \mathrm{e}^{\theta_{1} \tau}+\theta_{2} \mathrm{e}^{\theta_{2} \tau}}{\theta_{1} \mathrm{e}^{\theta_{1} \tau}+\mathrm{e}^{\theta_{2} \tau}},
$$

but due to the initial condition $C_{1}(0, \omega)=0$, we have $\theta_{3}=0$, and hence $C_{1}(\tau, \omega)=0$.

Calculation of $E_{1}(\tau, \omega)$

Equation (116) can be re-written as follows:

$$
\frac{\partial E_{1}}{\partial \tau}+\kappa_{\beta} E_{1}+\mathbb{E}[\sqrt{u}] \mathbb{E}[\sqrt{v}] i \omega=0,
$$

where we know that (see Proposition 1)

$$
\mathbb{E}[\sqrt{v}(t)] \approx m_{\mathrm{V}}+n_{\mathrm{V}} \mathrm{e}^{-l_{\mathrm{V}}(T-\tau)} \text { and } \mathbb{E}[\sqrt{u}(t)] \approx m_{\mathrm{U}}+n_{\mathrm{U}} \mathrm{e}^{-l_{\mathrm{U}}(T-\tau)} .
$$

Since

$$
\begin{aligned}
& \mathbb{E}[\sqrt{v}(t)] \mathbb{E}[\sqrt{u}(t)] \\
& =m_{\mathrm{V}} m_{\mathrm{U}}+m_{\mathrm{V}} n_{\mathrm{U}} \mathrm{e}^{-l_{\mathrm{U}}(T-\tau)}+m_{\mathrm{U}} n_{\mathrm{V}} \mathrm{e}^{-l_{\mathrm{V}}(T-\tau)}+n_{\mathrm{U}} n_{\mathrm{V}} \mathrm{e}^{-\left(l_{\mathrm{U}}+l_{\mathrm{V}}\right)(T-\tau)},
\end{aligned}
$$

the solution for the first-order ODE in $E_{1}$ reads as: 


$$
\begin{aligned}
E_{1}(\tau, \omega)= & (-i \omega) \mathrm{e}^{-\kappa_{\beta} \tau} \int_{0}^{\tau} \mathrm{e}^{\kappa_{\beta} s}\left(m_{\mathrm{V}} m_{\mathrm{U}}+m_{\mathrm{V}} n_{\mathrm{U}} \mathrm{e}^{-l_{\mathrm{U}}(T-s)}+m_{\mathrm{U}} n_{\mathrm{V}} \mathrm{e}^{-l_{\mathrm{V}}(T-s)}\right. \\
& \left.+n_{\mathrm{U}} n_{\mathrm{V}} \mathrm{e}^{-\left(l_{\mathrm{U}}+l_{\mathrm{V}}\right)(T-s)}\right) \mathrm{d} s .
\end{aligned}
$$

Further calculations show that

$$
\begin{aligned}
E_{1}(\tau, \omega)= & -i \omega\left[\frac{m_{\mathrm{V}} m_{\mathrm{U}}}{\kappa_{\beta}}\left(1-\mathrm{e}^{-\kappa_{\beta} \tau}\right)+\frac{m_{\mathrm{V}} n_{\mathrm{U}}}{\kappa_{\beta}+l_{\mathrm{U}}} \mathrm{e}^{-l_{\mathrm{U}}(T-\tau)}\left(1-\mathrm{e}^{-\left(\kappa_{\beta}+l_{\mathrm{U}}\right) \tau}\right)\right. \\
& +\frac{m_{\mathrm{U}} n_{\mathrm{V}}}{\kappa_{\beta}+l_{\mathrm{V}}} \mathrm{e}^{-l_{\mathrm{V}}(T-\tau)}\left(1-\mathrm{e}^{-\left(\kappa_{\beta}+l_{\mathrm{V}}\right) \tau}\right) \\
& \left.+\frac{n_{\mathrm{U}} n_{\mathrm{V}}}{\kappa_{\beta}+l_{\mathrm{U}}+l_{\mathrm{V}}} \mathrm{e}^{-\left(l_{\mathrm{U}}+l_{\mathrm{V}}\right)(T-\tau)}\left(1-\mathrm{e}^{-\left(\kappa_{\beta}+l_{\mathrm{U}}+l_{\mathrm{V}}\right) \tau}\right)\right] .
\end{aligned}
$$

Calculation of $F_{1}(\tau, \omega)$

We look then at Equation (117):

$$
\frac{\partial F_{1}}{\partial \tau}+\kappa_{\eta} F_{1}=\sigma_{\mathrm{v}} \mathbb{E}[v] B_{1}(i \omega+1),
$$

where we know that

$$
\mathbb{E}[v(t)]=\left(v(0)-\mu_{\mathrm{V}}\right) \mathrm{e}^{-\kappa_{\mathrm{V}}(T-\tau)}+\mu_{\mathrm{V}} \text { and } B_{1}(\tau, \omega)=\frac{\kappa_{\mathrm{V}}-\bar{B}_{1}}{\sigma_{\mathrm{V}}^{2}} \frac{1-\mathrm{e}^{-\bar{B}_{1} \tau}}{1-\bar{B}_{2} \mathrm{e}^{-\bar{B}_{1} \tau}} .
$$

As done in Teng et al. (2016) (Appendix B.1) we approximate the term

$$
\frac{1-\mathrm{e}^{-\bar{B}_{1} \tau}}{1-\bar{B}_{2} \mathrm{e}^{-\bar{B}_{1} \tau}} \approx 1-\mathrm{e}^{-\bar{B}_{3} \tau}, \text { with } \bar{B}_{3}=-\ln \left(\frac{\mathrm{e}^{-\bar{B}_{1}}-\bar{B}_{2} \mathrm{e}^{-\bar{B}_{1}}}{1-\bar{B}_{2} \mathrm{e}^{-\bar{B}_{1}}}\right),
$$

from which it follows that

$$
\frac{\partial F_{1}}{\partial \tau}+\kappa_{\eta} F_{1}=\frac{\kappa_{\mathrm{V}}-\bar{B}_{1}}{\sigma_{\mathrm{V}}}(i \omega+1)\left(1-\mathrm{e}^{-\bar{B}_{3} \tau}\right)\left(\mu_{\mathrm{V}}+\left(v(0)-\mu_{\mathrm{V}}\right) \mathrm{e}^{-\kappa_{\mathrm{V}}(T-\tau)}\right),
$$

whose solution reads as (since we have $F_{1}(0, \omega)=0$ ):

$$
\begin{aligned}
F_{1}(\tau, \omega)= & \frac{\left(\mu_{\mathrm{V}}-v(0)\right) \hat{B}_{1}}{\kappa_{\eta}+\kappa_{\mathrm{V}}-\bar{B}_{3}} \mathrm{e}^{\left(\kappa_{\mathrm{V}}-\bar{B}_{3}\right) \tau-\kappa_{\mathrm{V}} T}+\frac{\left(v(0)-\mu_{\mathrm{V}}\right) \hat{B}_{1}}{\kappa_{\eta}+\kappa_{\mathrm{V}}} \mathrm{e}^{\kappa_{\mathrm{V}}(\tau-T)} \\
& +\frac{\mu_{\mathrm{V}} \hat{B}_{1}}{\kappa_{\eta}}-\frac{\mu_{\mathrm{V}} \hat{B}_{1}}{\kappa_{\eta}-\bar{B}_{3}} \mathrm{e}^{-\bar{B}_{3} \tau}+\hat{B}_{1} \hat{B}_{2} \mathrm{e}^{-\kappa_{\eta} \tau}
\end{aligned}
$$

with

$$
\hat{B}_{1}=\frac{i \omega+1}{\sigma_{\mathrm{V}}}\left(\kappa_{\mathrm{V}}-\bar{B}_{1}\right)
$$

and

$$
\hat{B}_{2}=-\frac{\mu_{\mathrm{V}}}{\kappa_{\mathrm{V}}}-\frac{v(0)-\mu_{\mathrm{V}}}{\kappa_{\eta}+\kappa_{\mathrm{V}}} \mathrm{e}^{-\kappa_{\mathrm{V}} T}-\frac{\mu_{\mathrm{V}}-v(0)}{\kappa_{\eta}+\kappa_{\mathrm{V}}-\bar{B}_{3}} \mathrm{e}^{-\kappa_{\mathrm{V}} T}+\frac{\mu_{\mathrm{V}}}{\kappa_{\eta}-\bar{B}_{3}} .
$$

Calculation of $A_{1}(\tau, \omega)$

The last term to be computed is $A_{1}$ : 


$$
\begin{aligned}
A_{1}(\tau, \omega)= & r_{\mathrm{f}} i \omega \tau+\int_{0}^{\tau}\left(\kappa_{\eta} \mu_{\eta}+(i \omega+1) \rho_{\mathrm{S} \eta} \sigma_{\eta} \mathbb{E}[\sqrt{v(T-s)}]\right) F_{1}(s, \omega) \mathrm{d} s \\
& +\kappa_{\mathrm{V}} \mu_{\mathrm{V}} \int_{0}^{\tau} B_{1}(s, \omega) \mathrm{d} s+\int_{0}^{\tau}\left(\kappa_{\beta} \mu_{\beta}+(i \omega+1) \rho_{\mathrm{S} \beta} \sigma_{\beta} \mathbb{E}[\sqrt{v(T-s)}]\right) \\
& \times E_{1}(s, \omega) \mathrm{d} s+\frac{1}{2}\left[\sigma_{\beta}^{2} \int_{0}^{\tau} E_{1}^{2}(s, \omega) \mathrm{d} s+\sigma_{\eta}^{2} \int_{0}^{\tau} F_{1}^{2}(s, \omega) \mathrm{d} s\right]
\end{aligned}
$$

In particular, it can be proved (after tedious calculations) that

$$
\begin{aligned}
& \kappa_{\mathrm{V}} \mu_{\mathrm{V}} \int_{0}^{\tau} B_{1}(s, \omega) \mathrm{d} s=\frac{\kappa_{\mathrm{V}} \mu_{\mathrm{V}}}{\sigma_{\mathrm{V}}^{2}}\left(\left(\kappa_{\mathrm{V}}-\bar{B}_{1}\right) \tau-2 \ln \left(\frac{1-\bar{B}_{2} \mathrm{e}^{-\bar{B}_{1} \tau}}{1-\bar{B}_{2}}\right)\right), \\
& \kappa_{\eta} \mu_{\eta} \int_{0}^{\tau} F_{1}(s, \omega) \mathrm{d} s=\kappa_{\eta} \mu_{\eta}\left[\frac{\left(v(0)-\mu_{\mathrm{V}}\right) \hat{B}_{1} \mathrm{e}^{-\kappa_{\mathrm{V}} T}}{\kappa_{\eta}+\kappa_{\mathrm{V}}-\bar{B}_{3}} \frac{1-\mathrm{e}^{\left(\kappa_{\mathrm{V}}-\bar{B}_{3}\right) \tau}}{\kappa_{\mathrm{V}}-\bar{B}_{3}}\right. \\
& +\frac{\left(\mu_{\mathrm{V}}-v(0)\right) \hat{B}_{1} \mathrm{e}^{-\kappa_{\mathrm{V}} T}}{\kappa_{\eta}+\kappa_{\mathrm{V}}}\left(1-\mathrm{e}^{-\kappa_{\mathrm{V}} \tau}\right)+\frac{\mu_{\mathrm{V}} \hat{B}_{1}}{\kappa_{\eta}} \tau \\
& \left.-\frac{\mu_{\mathrm{V}} \hat{B}_{1}}{\bar{B}_{3}\left(\kappa_{\eta}-\bar{B}_{3}\right)}\left(1-\mathrm{e}^{-\bar{B}_{3} \tau}\right)+\frac{\hat{B}_{1} \hat{B}_{2}}{\kappa_{\eta}}\left(1-\mathrm{e}^{-\kappa_{\eta} \tau}\right)\right] \text {. }
\end{aligned}
$$

Besides that, we notice that

$$
\int_{0}^{\tau} \mathbb{E}[\sqrt{v(T-s)}] F_{1}(s, \omega) \mathrm{d} s \approx m_{\mathrm{V}} \int_{0}^{\tau} F_{1}(s, \omega) \mathrm{d} s+n_{\mathrm{V}} \int_{0}^{\tau} \mathrm{e}^{-l_{\mathrm{V}}(T-s)} F_{1}(s, \omega) \mathrm{d} s,
$$

where

$$
\begin{aligned}
& \int_{0}^{\tau} \mathrm{e}^{-l_{\mathrm{V}}(T-s)} F_{1}(s, \omega) \mathrm{d} s \\
& =\frac{\left(\mu_{\mathrm{V}}-v(0)\right) \mathrm{e}^{-\left(\kappa_{\mathrm{V}}+l_{\mathrm{V}}\right) T}}{\kappa_{\eta}+\kappa_{\mathrm{V}}-\bar{B}_{3}} \frac{\mathrm{e}^{\left(l_{\mathrm{V}}+\kappa_{\mathrm{V}}-\bar{B}_{3}\right) \tau}-1}{l_{\mathrm{V}}+\kappa_{\mathrm{V}}-\bar{B}_{3}} \\
& +\frac{\left(v(0)-\mu_{\mathrm{V}}\right) \mathrm{e}^{-\left(l_{\mathrm{V}}+\kappa_{\mathrm{V}}\right) T}}{\kappa_{\eta}+\kappa_{\mathrm{V}}} \frac{\mathrm{e}^{\left(\kappa_{\mathrm{V}}+l_{\mathrm{V}}\right) \tau}-1}{l_{\mathrm{V}}+\kappa_{\mathrm{V}}} \\
& +\frac{\mu_{\mathrm{V}} \hat{B}_{1} \mathrm{e}^{-l_{\mathrm{V}} T}}{\kappa_{\eta} l_{\mathrm{V}}}\left(\mathrm{e}^{l_{\mathrm{V}} \tau}-1\right)-\frac{\mu_{\mathrm{V}} \hat{B}_{1} \mathrm{e}^{-l_{\mathrm{V}} T}}{\kappa_{\eta}-\bar{B}_{3}} \frac{\mathrm{e}^{\left(l_{\mathrm{V}}-\bar{B}_{3}\right) \tau}-1}{l_{\mathrm{V}}-\bar{B}_{3}} \\
& +\frac{\hat{B}_{1} \hat{B}_{2} \mathrm{e}^{-l_{\mathrm{V}} T}}{l_{\mathrm{V}}-\kappa_{\eta}}\left(\mathrm{e}^{\left(l_{\mathrm{V}}-\kappa_{\eta}\right) \tau}-1\right) .
\end{aligned}
$$

Now, further calculations show that

$$
\begin{aligned}
& \kappa_{\beta} \mu_{\beta} \int_{0}^{\tau} E_{1}(s, \omega) \mathrm{d} s \\
& =-i \omega \kappa_{\beta} \mu_{\beta}\left[\frac{m_{\mathrm{U}} m_{\mathrm{V}}}{\kappa_{\beta}}\left(\tau+\frac{\mathrm{e}^{-\kappa_{\beta} \tau}-1}{\kappa_{\beta}}\right)+\frac{m_{\mathrm{U}} m_{\mathrm{V}}}{\kappa_{\beta}+l_{\mathrm{U}}} \mathrm{e}^{-l_{\mathrm{U}} T}\left(\frac{\mathrm{e}^{l_{\mathrm{U}} \tau}-1}{l_{\mathrm{U}}}\right.\right. \\
& \left.+\frac{\mathrm{e}^{-\kappa_{\beta} \tau}-1}{\kappa_{\beta}}\right)+\frac{m_{\mathrm{U}} n_{\mathrm{V}}}{\kappa_{\mathrm{b}}+l_{\mathrm{V}}} \mathrm{e}^{-l_{\mathrm{V}} T}\left(\frac{\mathrm{e}^{l_{\mathrm{V} \tau}}-1}{l_{\mathrm{V}}}+\frac{\mathrm{e}^{-\kappa_{\beta} \tau}-1}{\kappa_{\beta}}\right) \\
& \left.+\frac{n_{\mathrm{U}} n_{\mathrm{V}}}{\kappa_{\beta}+l_{\mathrm{U}}+l_{\mathrm{V}}} \mathrm{e}^{-\left(l_{\mathrm{U}}+l_{\mathrm{V}}\right) T}\left(\frac{\mathrm{e}^{\left(l_{\mathrm{U}}+l_{\mathrm{V}}\right) \tau}-1}{l_{\mathrm{U}}+l_{\mathrm{V}}}+\frac{\mathrm{e}^{-\kappa_{\beta} \tau}-1}{\kappa_{\beta}}\right)\right],
\end{aligned}
$$

and 


$$
\int_{0}^{\tau} \mathbb{E}[\sqrt{v(T-s)}] E_{1}(s, \omega) \mathrm{d} s \approx m_{\mathrm{V}} \int_{0}^{\tau} E_{1}(s, \omega) \mathrm{d} s+n_{\mathrm{V}} \int_{0}^{\tau} \mathrm{e}^{-l_{\mathrm{v}}(T-s)} E_{1}(s, \omega) \mathrm{d} s,
$$

where

$$
\begin{aligned}
& \int_{0}^{\tau} \mathrm{e}^{-l_{\mathrm{V}}(T-s)} E_{1}(s, \omega) \mathrm{d} s \\
& =-(i \omega)\left[\frac{m_{\mathrm{V}} m_{\mathrm{U}}}{\kappa_{\beta}} \mathrm{e}^{-l_{\mathrm{V}} T}\left(\frac{\mathrm{e}^{l_{\mathrm{V}} \tau}-1}{l_{\mathrm{V}}}-\frac{\mathrm{e}^{\left(l_{\mathrm{V}}-\kappa_{\beta}\right) \tau}-1}{l_{\mathrm{V}}-\kappa_{\beta}}\right)\right. \\
& +\frac{m_{\mathrm{V}} n_{\mathrm{U}}}{\kappa_{\beta}+l_{\mathrm{U}}} \mathrm{e}^{-\left(l_{\mathrm{U}}+l_{\mathrm{V}}\right) T}\left(\frac{\mathrm{e}^{\left(l_{\mathrm{U}}+l_{\mathrm{V}}\right) \tau}-1}{l_{\mathrm{U}}+l_{\mathrm{V}}}-\frac{\mathrm{e}^{\left(l_{\mathrm{V}}-\kappa_{\beta}\right) \tau}-1}{l_{\mathrm{V}}-\kappa_{\beta}}\right) \\
& +\frac{m_{\mathrm{U}} n_{\mathrm{V}}}{\kappa_{\beta}+l_{\mathrm{V}}} \mathrm{e}^{-2 l_{\mathrm{V}} T}\left(\frac{\mathrm{e}^{2 l_{\mathrm{V}} \tau}-1}{2 l_{\mathrm{V}}}-\frac{\mathrm{e}^{\left(l_{\mathrm{V}}-\kappa_{\beta}\right) \tau}-1}{l_{\mathrm{V}}-\kappa_{\beta}}\right) \\
& \left.+\frac{n_{\mathrm{U}} n_{\mathrm{V}}}{\kappa_{\beta}+l_{\mathrm{U}}+l_{\mathrm{V}}} \mathrm{e}^{-\left(l_{\mathrm{U}}+2 l_{\mathrm{V}}\right) T}\left(\frac{\mathrm{e}^{\left(l_{\mathrm{U}}+2 l_{\mathrm{V}}\right) \tau}-1}{l_{\mathrm{U}}+2 l_{\mathrm{V}}}-\frac{\mathrm{e}^{\left(l_{\mathrm{V}}-\kappa_{\beta}\right) \tau}-1}{l_{\mathrm{V}}-\kappa_{\beta}}\right)\right] .
\end{aligned}
$$

Finally, it can be proved that

$$
\begin{aligned}
\int_{0}^{\tau} E_{1}(s, \omega)^{2} \mathrm{~d} s & -\omega^{2}\left[\frac{m_{\mathrm{V}}^{2} m_{\mathrm{U}}^{2}}{\kappa_{\beta}^{2}}\left(\tau+\frac{1-\mathrm{e}^{-2 \kappa_{\beta} \tau}}{2 \kappa_{\beta}}-\frac{2}{\kappa_{\beta}}\left(1-\mathrm{e}^{-\kappa_{\beta} \tau}\right)\right)+\frac{m_{\mathrm{V}}^{2} n_{\mathrm{U}}^{2}}{\left(\kappa_{\beta}+\kappa_{\mathrm{U}}\right)^{2}} \mathrm{e}^{-2 l_{\mathrm{U}} T}\right. \\
& \times\left(\frac{1}{2 l_{\mathrm{U}}}\left(\mathrm{e}^{2 l_{\mathrm{U}} \tau}-1\right)-\frac{1}{2 \kappa_{\beta}}\left(\mathrm{e}^{-2 \kappa_{\beta} \tau}-1\right)-\frac{2}{\kappa_{\beta}}\left(\mathrm{e}^{\kappa_{\beta} \tau}-1\right)\right) \\
& +\frac{m_{\mathrm{U}}^{2} n_{\mathrm{V}}^{2}}{\left(\kappa_{\beta}+l_{\mathrm{V}}\right)^{2}} \mathrm{e}^{-2 l_{\mathrm{V}} T}\left(\frac{\mathrm{e}^{2 l_{\mathrm{V}} \tau}-1}{2 l_{\mathrm{V}}}-\frac{1}{2 \kappa_{\beta}}\left(\mathrm{e}^{-2 \kappa_{\beta} \tau}-1\right)\right. \\
& \left.-\frac{2}{l_{\mathrm{V}}-\kappa_{\beta}}\left(\mathrm{e}^{\left(l_{\mathrm{V}}-\kappa_{\beta}\right) \tau}-1\right)\right)+\frac{n_{\mathrm{U}}^{2} n_{\mathrm{V}}^{2}}{\left(\kappa_{\beta}+l_{\mathrm{U}}+l_{\mathrm{V}}\right)^{2}} \mathrm{e}^{-2\left(l_{\mathrm{U}}+l_{\mathrm{V}}\right) T}\left(\frac{\mathrm{e}^{2\left(l_{\mathrm{U}}+l_{\mathrm{V}}\right) \tau}-1}{2\left(l_{\mathrm{U}}+l_{\mathrm{V}}\right)}\right. \\
- & \left.\frac{\mathrm{e}^{-2 \kappa_{\beta} \tau}-1}{2 \kappa_{\beta}}-\frac{2}{l_{\mathrm{V}}+l_{\mathrm{U}}-\kappa_{\beta}}\left(\mathrm{e}^{\left(l_{\mathrm{V}}+l_{\mathrm{U}}-\kappa_{\beta}\right) \tau}-1\right)\right) \\
+ & 2 \frac{m_{\mathrm{V}}^{2} m_{\mathrm{U}} n_{\mathrm{U}}}{\kappa_{\beta}\left(\kappa_{\beta}+l_{\mathrm{U}}\right)} \mathrm{e}^{-l_{\mathrm{U}} T}\left(\frac{\mathrm{e}^{l_{\mathrm{U}} \tau}-1}{l_{\mathrm{U}}}+\frac{\mathrm{e}^{-\kappa_{\beta}}-1}{\kappa_{\beta}}-\frac{\mathrm{e}^{\left(l_{\mathrm{U}}-\kappa_{\beta}\right)}-1}{l_{\mathrm{U}}-\kappa_{\beta}}-\frac{\mathrm{e}^{-2 \kappa_{\beta} \tau}-1}{2 \kappa_{\beta}}\right) \\
+ & 2 \frac{m_{\mathrm{V}} m_{\mathrm{U}}^{2} n_{\mathrm{V}}}{\kappa_{\beta}\left(\kappa_{\beta}+l_{\mathrm{V}}\right)} \mathrm{e}^{-l_{\mathrm{V}} T}\left(\frac{\mathrm{e}^{l_{\mathrm{V}} \tau}-1}{l_{\mathrm{V}}}+\frac{\mathrm{e}^{-\kappa_{\beta} \tau}-1}{\kappa_{\beta}}-\frac{\mathrm{e}^{\left(l_{\mathrm{V}}-\kappa_{\beta}\right) \tau}-1}{l_{\mathrm{V}}-\kappa_{\beta}}-\frac{\mathrm{e}^{-2 \kappa_{\beta} \tau}-1}{2 \kappa_{\beta}}\right) \\
+ & 2 \frac{m_{\mathrm{V}} m_{\mathrm{U}} n_{\mathrm{V}} n_{\mathrm{U}}}{\kappa_{\beta}\left(\kappa_{\beta}+l_{\mathrm{U}}+l_{\mathrm{V}}\right)} \mathrm{e}^{-\left(l_{\mathrm{U}}+l_{\mathrm{V}}\right) T}-\frac{\mathrm{e}^{\left(l_{\mathrm{U}}+l_{\mathrm{V}}\right) \tau}-1}{l_{\mathrm{U}}+l_{\mathrm{V}}}-\frac{\mathrm{e}^{\left(l_{\mathrm{U}}+l_{\mathrm{V}}-\kappa_{\beta}\right) \tau}-1}{l_{\mathrm{U}}+l_{\mathrm{V}}-\kappa_{\beta}}+\frac{\mathrm{e}^{-\kappa_{\beta} \tau}-1}{\kappa_{\beta}}+2 \frac{m_{\mathrm{V}} n_{\mathrm{U}} m_{\mathrm{U}} n_{\mathrm{V}}}{\left(\kappa_{\beta}+l_{\mathrm{U}}\right)\left(\kappa_{\beta}+l_{\mathrm{V}}\right)} \mathrm{e}^{-\left(l_{\mathrm{U}}+l_{\mathrm{V}}\right) T}\left(\frac{\mathrm{e}^{\left(l_{\mathrm{U}}+l_{\mathrm{V}}\right) \tau}-1}{l_{\mathrm{U}}+l_{\mathrm{V}}}-\frac{\mathrm{e}^{\left(l_{\mathrm{V}}-\kappa_{\beta}\right) \tau}-1}{l_{\mathrm{V}}-\kappa_{\beta}}\right.
\end{aligned}
$$




$$
\begin{aligned}
& \left.-\frac{\mathrm{e}^{\left(l_{\mathrm{U}}-\kappa_{\beta}\right) \tau}-1}{l_{\mathrm{U}}-\kappa_{\beta}}-\frac{\mathrm{e}^{-2 \kappa_{\beta} \tau}-1}{2 \kappa_{\beta}}\right)+2 \frac{m_{\mathrm{V}} n_{\mathrm{U}}^{2} n_{\mathrm{V}}}{\left(\kappa_{\beta}+l_{\mathrm{U}}\right)\left(\kappa_{\beta}+l_{\mathrm{U}}+l_{\mathrm{V}}\right)} \mathrm{e}^{-\left(2 l_{\mathrm{U}}+l_{\mathrm{V}}\right) T} \\
& \times\left(\frac{\mathrm{e}^{\left(2 l_{\mathrm{U}}+l_{\mathrm{V}}\right) \tau}-1}{2 l_{\mathrm{U}}+l_{\mathrm{V}}}-\frac{\mathrm{e}^{\left(l_{\mathrm{U}}-\kappa_{\beta}\right) \tau}-1}{l_{\mathrm{U}}-\kappa_{\beta}}-\frac{\mathrm{e}^{\left(l_{\mathrm{U}}+l_{\mathrm{V}}-\kappa_{\beta}\right) \tau}-1}{l_{\mathrm{U}}+l_{\mathrm{V}}-\kappa_{\beta}}-\frac{\mathrm{e}^{-2 \kappa_{\beta} \tau}-1}{2 \kappa_{\beta}}\right) \\
& +2 \frac{m_{\mathrm{U}} n_{\mathrm{U}} n_{\mathrm{V}}^{2}}{\left(\kappa_{\beta}+l_{\mathrm{V}}\right)\left(\kappa_{\beta}+l_{\mathrm{U}}+l_{\mathrm{V}}\right)} \mathrm{e}^{-\left(l_{\mathrm{U}}+2 l_{\mathrm{V}}\right) T}\left(\frac{\mathrm{e}^{\left(l_{\mathrm{U}}+2 l_{\mathrm{V}}\right) \tau}-1}{l_{\mathrm{U}}+2 l_{\mathrm{V}}}-\frac{\mathrm{e}^{\left(l_{\mathrm{U}}+l_{\mathrm{V}}-\kappa_{\beta}\right) \tau}-1}{l_{\mathrm{U}}+l_{\mathrm{V}}-\kappa_{\beta}}\right. \\
& \left.\left.-\frac{\mathrm{e}^{\left(l_{\mathrm{V}}-\kappa_{\beta}\right) \tau}-1}{l_{\mathrm{V}}-\kappa_{\beta}}-\frac{\mathrm{e}^{-2 \kappa_{\beta} \tau}-1}{2 \kappa_{\beta}}\right)\right],
\end{aligned}
$$

and that

$$
\begin{aligned}
& \int_{0}^{\tau} F_{1}(s, \omega)^{2} \mathrm{~d} s \\
& =\frac{\left(\mu_{\mathrm{V}}-v(0)\right)^{2}}{\left(\kappa_{\eta}+\kappa_{\mathrm{V}}-\bar{B}_{3}\right)^{2}} \hat{B}_{1}^{2} \mathrm{e}^{-2 \kappa_{\mathrm{V}} T} \frac{\mathrm{e}^{2\left(\kappa_{\mathrm{V}}-\bar{B}_{3}\right) \tau}-1}{2\left(\kappa_{\mathrm{V}}-\bar{B}_{3}\right)}+\frac{\left(v(0)-\mu_{\mathrm{V}}\right)^{2}}{\left(\kappa_{\eta}+\kappa_{\mathrm{V}}\right)^{2}} \hat{B}_{1}^{2} \mathrm{e}^{-2 \kappa_{\mathrm{V}} T} \frac{\mathrm{e}^{2 \kappa_{\mathrm{V}} \tau}-1}{2 \kappa_{\mathrm{V}}} \\
& +\frac{\mu_{\mathrm{V}}^{2} \hat{B}_{1}^{2}}{\kappa_{\eta}^{2}} \tau-\frac{\mu_{\mathrm{V}}^{2} \hat{B}_{1}^{2}}{\left(\kappa_{\eta}-\bar{B}_{3}\right)^{2}} \frac{\mathrm{e}^{-2 \bar{B}_{3} \tau}-1}{2 \bar{B}_{3}}-\frac{\hat{B}_{1}^{2} \hat{B}_{2}^{2}}{2 \kappa_{\eta}}\left(\mathrm{e}^{-2 \kappa_{\eta} \tau}-1\right) \\
& +2 \frac{\left(\mu_{\mathrm{V}}-v(0)\right)\left(v(0)-\mu_{\mathrm{V}}\right) \hat{B}_{1}^{2}}{\left(\kappa_{\eta}+\kappa_{\mathrm{V}}-\bar{B}_{3}\right)\left(\kappa_{\mathrm{V}}+\kappa_{\eta}\right)} \frac{\mathrm{e}^{2\left(\kappa_{\mathrm{V}}-\bar{B}_{3}\right) \tau}-1}{2\left(\kappa_{\mathrm{V}}-\bar{B}_{3}\right)}+2 \frac{\mu_{\mathrm{V}}\left(\mu_{\mathrm{V}}-v(0)\right)}{\kappa_{\eta}\left(\kappa_{\eta}+\kappa_{\mathrm{V}}-\bar{B}_{3}\right)} \hat{B}_{1}^{2} \mathrm{e}^{-\kappa_{\mathrm{V}} T} \\
& \times \frac{\mathrm{e}^{\left(\kappa_{\mathrm{V}}-\bar{B}_{3}\right) \tau}-1}{\kappa_{\mathrm{V}}-\bar{B}_{3}}-2 \frac{\mu_{\mathrm{V}}\left(\mu_{\mathrm{V}}-v(0)\right) \hat{B}_{1} \mathrm{e}^{-\kappa_{\mathrm{V}} T}}{\left(\kappa_{\eta}-\bar{B}_{3}\right)\left(\kappa_{\eta}+\kappa_{\mathrm{V}}-\bar{B}_{3}\right)} \frac{\mathrm{e}^{\left(\kappa_{\mathrm{V}}-2 \bar{B}_{3}\right) \tau}-1}{\kappa_{\mathrm{V}}-2 \bar{B}_{3}} \\
& +2 \frac{\left(\mu_{\mathrm{V}}-v(0)\right) \hat{B}_{1}^{2} \hat{B}_{2} \mathrm{e}^{-\kappa_{\mathrm{V}} T}}{\kappa_{\eta}+\kappa_{\mathrm{V}}-\bar{B}_{3}} \frac{\mathrm{e}^{\left(\kappa_{\mathrm{V}}-\bar{B}_{3}-\kappa_{\eta}\right) \tau}-1}{\kappa_{\mathrm{V}}-\bar{B}_{3}-\kappa_{\eta}}+2 \frac{\mu_{\mathrm{V}}\left(v(0)-\mu_{\mathrm{V}}\right) \hat{B}_{1}^{2} \mathrm{e}^{-\kappa_{\mathrm{V}} T}}{\kappa_{\eta}\left(\kappa_{\eta}+\kappa_{\mathrm{V}}\right)} \frac{\mathrm{e}^{\kappa_{\mathrm{V}} \tau}-1}{\kappa_{\mathrm{V}}} \\
& -2 \frac{\mu_{\mathrm{V}}\left(v(0)-\mu_{\mathrm{V}}\right) \hat{B}_{1}^{2} \mathrm{e}^{-\kappa_{\mathrm{V}} T}}{\left(\kappa_{\eta}+\kappa_{\mathrm{V}}\right)\left(\kappa_{\eta}-\bar{B}_{3}\right)} \frac{\mathrm{e}^{\left(\kappa_{\mathrm{V}}-\bar{B}_{3}\right) \tau}-1}{\kappa_{\mathrm{V}}-\bar{B}_{3}}+2 \frac{\left(v(0)-\mu_{\mathrm{V}}\right) \hat{B}_{1}^{2} \hat{B}_{2} \mathrm{e}^{-\kappa_{\mathrm{V}} T}}{\kappa_{\mathrm{V}}+\kappa_{\eta}} \frac{\mathrm{e}^{\left(\kappa_{\mathrm{V}}-\kappa_{\eta}\right)}-1}{\kappa_{\mathrm{V}}-\kappa_{\eta}} \\
& -2 \frac{\mu_{\mathrm{V}}^{2} \hat{B}_{1}^{2}}{\kappa_{\eta}\left(\kappa_{\eta}-\bar{B}_{3}\right)} \frac{\mathrm{e}^{-\bar{B}_{3} \tau}-1}{\bar{B}_{3}}-2 \frac{\mu_{\mathrm{V}} \hat{B}_{1}^{2} \hat{B}_{2}}{\kappa_{\eta}} \frac{\mathrm{e}^{-\kappa_{\eta} \tau}-1}{\kappa_{\eta}}+2 \frac{\mu_{\mathrm{V}} \hat{B}_{1}^{2} \hat{B}_{2}}{\kappa_{\eta}-\bar{B}_{3}} \frac{\mathrm{e}^{-\bar{B}_{3} \tau}-1}{\bar{B}_{3}} .
\end{aligned}
$$

Collecting all the terms together completes the proof.

\section{A.3. The Proof of Lemma 4}

Since the system generating the pricing PDE for $P_{1}$ (75) is of affine form, we look for a solution $\phi_{1}=\phi_{1}(\tau, \omega)$ of the following form:

$$
\begin{aligned}
\phi_{1}^{\mathrm{BJ}}= & \exp \left(T_{1}(\tau, \omega)+L_{1}(\tau, \omega) v(t)+M_{1}(\tau, \omega) u(t)+N_{1}(\tau, \omega) z(t)\right. \\
& \left.+Q_{1}(\tau, \omega) \beta(t)+R_{1}(\tau, \omega) \eta(t)+S_{1}(\tau, \omega) \gamma(t)+i \omega y(t)\right),
\end{aligned}
$$

with

$$
T_{1}(0, \omega)=L_{1}(0, \omega)=M_{1}(0, \omega)=N_{1}(0, \omega)=Q_{1}(0, \omega)=R_{1}(0, \omega)=S_{1}(0, \omega)=0
$$

Substituting all the partial derivatives of $\phi_{1}$ into (75) gives: 


$$
\begin{aligned}
& -\left(\frac{\partial T_{1}}{\partial \tau}+v \frac{\partial L_{1}}{\partial \tau}+u \frac{\partial M_{1}}{\partial \tau}+z \frac{\partial N_{1}}{\partial \tau}+\beta \frac{\partial Q 1}{\partial \tau}+\eta \frac{\partial R_{1}}{\partial \tau}+\gamma \frac{\partial S_{1}}{\partial \tau}\right) \\
& +\left(r_{\mathrm{f}}+\frac{1}{2} v-\beta \mathbb{E}[\sqrt{v}] \mathbb{E}[\sqrt{u}]\right) i \omega+\left(r_{\mathrm{d}}-r_{\mathrm{f}}-\frac{1}{2} u+\beta \mathbb{E}[\sqrt{v}] \mathbb{E}[\sqrt{u}]\right) N_{1} \\
& +\left(\kappa_{\eta}\left(\mu_{\eta}-\eta\right)+\rho_{\mathrm{S} \eta} \sigma_{\eta} \mathbb{E}[\sqrt{v}] \mathbb{E}\left[\sqrt{1-\eta^{2}}\right]\right) R_{1}+\left(\kappa_{\mathrm{V}}\left(\mu_{\mathrm{v}}-v\right)+\sigma_{\mathrm{v}} \mathbb{E}[v] \eta\right) L_{1} \\
& +\kappa_{\mathrm{U}}\left(\mu_{\mathrm{U}}-u\right) M_{1}+\left(\kappa_{\beta}\left(\mu_{\beta}-\beta\right)+\rho_{\mathrm{S} \beta} \sigma_{\beta} \mathbb{E}[\sqrt{v}] \mathbb{E}\left[\sqrt{1-\beta^{2}}\right]\right) Q_{1} \\
& +\kappa_{\gamma}\left(\mu_{\gamma}-\gamma\right) S_{1}-\frac{1}{2} \omega^{2} v+\frac{1}{2} u N_{1}^{2}+\frac{1}{2} \sigma_{\mathrm{v}}^{2} v L_{1}^{2}+\frac{1}{2} \sigma_{\mathrm{U}}^{2} u M_{1}^{2}+\frac{1}{2} \sigma_{\beta}^{2}\left(1-\mathbb{E}\left[\beta^{2}\right]\right) Q_{1}^{2} \\
& +\frac{1}{2} \sigma_{\eta}^{2}\left(1-\mathbb{E}\left[\eta^{2}\right]\right) R_{1}^{2}+\frac{1}{2} \sigma_{\gamma}^{2}\left(1-\mathbb{E}\left[\gamma^{2}\right]\right) S_{1}^{2}+\beta \mathbb{E}[\sqrt{v}][\sqrt{u}] i \omega N_{1} \\
& +\sigma_{\mathrm{v}} \mathbb{E}[v] \eta i \omega L_{1}+\sigma_{\mathrm{U}} \mathbb{E}[u] \gamma N_{1} M_{1}+\rho_{\mathrm{S} \beta} \sigma_{\beta} \mathbb{E}[\sqrt{v}] \mathbb{E}\left[\sqrt{1-\beta^{2}}\right] i \omega Q_{1} \\
& +\rho_{\mathrm{S} \eta} \sigma_{\eta} \mathbb{E}[\sqrt{v}] \mathbb{E}\left[\sqrt{1-\eta^{2}}\right] i \omega R_{1}+\rho_{X \beta} \sigma_{\beta} \mathbb{E}[\sqrt{u}] \mathbb{E}\left[\sqrt{1-\beta^{2}}\right] N_{1} Q_{1} \\
& +\rho_{\mathrm{X} \gamma} \sigma_{\gamma} \mathbb{E}[\sqrt{u}] \mathbb{E}\left[\sqrt{1-\gamma^{2}}\right] N_{1} S_{1}=0 .
\end{aligned}
$$

Now, collecting together homogenous terms in $y, z, v, u, \beta, \eta, \gamma$ gives the following system of equations:

$$
\begin{gathered}
\frac{\partial L_{1}}{\partial \tau}+\kappa_{\mathrm{V}} L_{1}-\frac{1}{2} \sigma_{\mathrm{V}}^{2} L_{1}^{2}+\frac{1}{2} \omega(\omega-i)=0, \\
\frac{\partial M_{1}}{\partial \tau}+\kappa_{\mathrm{U}} M_{1}-\frac{1}{2} \sigma_{\mathrm{U}}^{2} M_{1}^{2}-\frac{1}{2} N_{1}\left(N_{1}-1\right)=0, \\
\frac{\partial N_{1}}{\partial \tau}=0, \\
\frac{\partial Q_{1}}{\partial \tau}+\kappa_{\beta} Q_{1}-\mathbb{E}[\sqrt{u}] \mathbb{E}[\sqrt{v}]\left(i \omega\left(N_{1}-1\right)+N_{1}\right)=0, \\
\frac{\partial R_{1}}{\partial \tau}+\kappa_{\eta} R_{1}-\sigma_{\mathrm{V}} \mathbb{E}[v] L_{1}(i \omega+1)=0, \\
\frac{\partial S_{1}}{\partial \tau}+\kappa_{\gamma} S_{1}-\sigma_{\mathrm{U}} \mathbb{E}[u] N_{1} M_{1}=0,
\end{gathered}
$$

and

$$
\begin{aligned}
\frac{\partial T_{1}}{\partial \tau}= & r_{\mathrm{f}} i \omega+\left(\kappa_{\eta} \mu_{\eta}+\rho_{\mathrm{S} \eta} \sigma_{\eta} \mathbb{E}[\sqrt{v}] \mathbb{E}\left[\sqrt{1-\eta^{2}}\right](i \omega+1)\right) R_{1}+\kappa_{\mathrm{V}} \mu_{\mathrm{v}} L_{1} \\
& +\kappa_{\mathrm{U}} \mu_{\mathrm{U}} M_{1}+\left(\kappa_{\beta} \mu_{\beta}+\rho_{\mathrm{S} \beta} \sigma_{\beta} \mathbb{E}[\sqrt{v}] \mathbb{E}\left[\sqrt{1-\beta^{2}}\right](i \omega+1)\right) Q_{1} \\
& +\kappa_{\gamma} \mu_{\gamma} S_{1}+\frac{1}{2} \sigma_{\beta}^{2}\left(1-\mathbb{E}\left[\beta^{2}\right]\right) Q_{1}^{2}+\frac{1}{2} \sigma_{\eta}^{2}\left(1-\mathbb{E}\left[\eta^{2}\right]\right) R_{1}^{2} \\
& +\frac{1}{2} \sigma_{\gamma}^{2}\left(1-\mathbb{E}\left[\gamma^{2}\right]\right) S_{1}^{2}+\rho_{X \beta} \sigma_{\beta} \mathbb{E}[\sqrt{u}] \mathbb{E}\left[\sqrt{1-\beta^{2}}\right] N_{1} Q_{1} \\
& +\rho_{\mathrm{x} \gamma} \sigma_{\gamma} \mathbb{E}[\sqrt{u}] \mathbb{E}\left[\sqrt{1-\gamma^{2}}\right] N_{1} S_{1}+\left(r_{\mathrm{d}}-r_{\mathrm{f}}\right) N_{1} .
\end{aligned}
$$

If we compare Equations (146)-(151) with Equations (113)-(118), we can conclude that 


$$
\begin{aligned}
& L_{1}(\tau, \omega) \equiv B_{1}(\tau, \omega), M_{1}(\tau, \omega) \equiv C_{1}(\tau, \omega), N_{1}(\tau, \omega) \equiv D_{1}(\tau, \omega), \\
& Q_{1}(\tau, \omega) \equiv E_{1}(\tau, \omega), R(\tau, \omega) \equiv F_{1}(\tau, \omega) \text { and } G_{1}(\tau, \omega) \equiv S_{1}(\tau, \omega),
\end{aligned}
$$

and therefore the only term to be calculated is $T_{1}(\tau, \omega)$, which can be re-written as follows:

$$
\begin{aligned}
T_{1}(\tau, \omega)= & { }_{\mathrm{f}} i \omega \tau+\int_{0}^{\tau}\left(\kappa_{\eta} \mu_{\eta}+(i \omega+1) \rho_{\mathrm{S} \eta} \sigma_{\eta} \mathbb{E}[\sqrt{v(T-s)}] \mathbb{E}\left[\sqrt{1-\eta(T-s)^{2}}\right]\right) \\
& \times F_{1}(s, \omega) \mathrm{d} s+\kappa_{\mathrm{V}} \mu_{\mathrm{V}} \int_{0}^{\tau} B_{1}(s, \omega) \mathrm{d} s \\
& +\int_{0}^{\tau}\left(\kappa_{\beta} \mu_{\beta}+(i \omega+1) \rho_{\mathrm{S} \beta} \sigma_{\beta} \mathbb{E}[\sqrt{v(T-s)}]\left[\sqrt{1-\beta(T-s)^{2}}\right]\right) \\
& \times E_{1}(s, \omega) \mathrm{d} s+\frac{1}{2}\left[\sigma_{\beta}^{2} \int_{0}^{\tau}\left(1-\mathbb{E}\left[\beta(T-s)^{2}\right]\right) E_{1}^{2}(s, \omega) \mathrm{d} s\right. \\
& \left.+\sigma_{\eta}^{2} \int_{0}^{\tau}\left(1-\mathbb{E}\left[\eta(T-s)^{2}\right]\right) F_{1}^{2}(s, \omega) \mathrm{d} s\right] .
\end{aligned}
$$

Now, from Propositions 1,2 , and 3 respectively, we know that $\mathbb{E}[\sqrt{v}], \mathbb{E}\left[\rho^{2}\right]$, and $\mathbb{E}\left[\sqrt{1-\rho^{2}}\right]$, with $\rho \in\{\beta, \eta\}$, can be approximated as follows:

$$
\begin{gathered}
\mathbb{E}[\sqrt{v(T-s)}] \approx m_{\mathrm{V}}+n_{\mathrm{V}} \mathrm{e}^{-l_{\mathrm{V}}(T-s)}, \\
\mathbb{E}\left[\sqrt{1-\beta(T-s)^{2}}\right] \approx \mathrm{e}^{-\hat{s}_{\beta}(T-s)}+\hat{r}_{\beta} \mathrm{e}^{-\hat{p}_{\beta}(T-s)}+\hat{q}_{\beta}, \\
\mathbb{E}\left[\sqrt{1-\eta(T-s)^{2}}\right] \approx \mathrm{e}^{-\hat{s}_{\eta}(T-s)}+\hat{r}_{\eta} \mathrm{e}^{-\hat{p}_{\eta}(T-s)}+\hat{q}_{\eta}, \\
\mathbb{E}\left[\beta(T-s)^{2}\right] \approx \mathrm{e}^{-s_{\beta}(T-s)}+r_{\beta} \mathrm{e}^{-p_{\beta}(T-s)}+q_{\beta}, \\
\mathbb{E}\left[\eta(T-s)^{2}\right] \approx \mathrm{e}^{-s_{\eta}(T-s)}+r_{\eta} \mathrm{e}^{-p_{\eta}(T-s)}+q_{\eta},
\end{gathered}
$$

where the coefficients $m_{\mathrm{V}}, n_{\mathrm{V}}, l_{\mathrm{V}}, s_{\rho}, r_{\rho}, p_{\rho}, q_{\rho}, \hat{s}_{\rho}, \hat{r}_{\rho}, \hat{p}_{\rho}, \hat{q}_{\rho}$, for $\rho \in\{\beta, \eta\}$, are defined in the relevant propositions.

Therefore, further calculations show that

$$
\begin{aligned}
& \int_{0}^{\tau} \mathbb{E}[\sqrt{v(T-s)}] \mathbb{E}\left[\sqrt{1-\eta^{2}(T-s)}\right] F_{1}(s, \omega) \mathrm{d} s \\
& =m_{\mathrm{V}} \int_{0}^{\tau} \mathrm{e}^{-\hat{s}_{\eta}(T-s)} F_{1}(s, \omega) \mathrm{d} s+m_{\mathrm{V}} \hat{r}_{\eta} \int_{0}^{\tau} \mathrm{e}^{-\hat{p}_{\eta}(T-s)} F_{1}(s, \omega) \mathrm{d} s \\
& +m_{\mathrm{V}} \hat{q}_{\eta} \int_{0}^{\tau} F_{1}(s, \omega) \mathrm{d} s+n_{\mathrm{V}} \int_{0}^{\tau} \mathrm{e}^{-\left(l_{\mathrm{V}}+\hat{s}_{\eta}\right)(T-s)} F_{1}(s, \omega) \mathrm{d} s \\
& +n_{\mathrm{V}} \hat{r}_{\eta} \int_{0}^{\tau} \mathrm{e}^{-\left(l_{\mathrm{V}}+\hat{p}_{\eta}\right)(T-s)} F_{1}(s, \omega) \mathrm{d} s+n_{\mathrm{V}} \hat{q}_{\eta} \int_{0}^{\tau} \mathrm{e}^{-l_{\mathrm{V}}(T-s)} F_{1}(s, \omega) \mathrm{d} s .
\end{aligned}
$$

Repeating the same calculation for the same integral with $\beta$ and $E_{1}$ instead of $\eta$ and $F_{1}$ gives

$$
\begin{aligned}
& \int_{0}^{\tau} \mathbb{E}[\sqrt{v(T-s)}] \mathbb{E}\left[\sqrt{1-\beta^{2}(T-s)}\right] E_{1}(s, \omega) \mathrm{d} s \\
& =m_{\mathrm{V}} \int_{0}^{\tau} \mathrm{e}^{-\hat{s}_{\beta}(T-s)} E_{1}(s, \omega) \mathrm{d} s+m_{\mathrm{V}} \hat{r}_{\beta} \int_{0}^{\tau} \mathrm{e}^{-\hat{p}_{\beta}(T-s)} E_{1}(s, \omega) \mathrm{d} s \\
& +m_{\mathrm{V}} \hat{q}_{\beta} \int_{0}^{\tau} E_{1}(s, \omega) \mathrm{d} s+n_{\mathrm{V}} \int_{0}^{\tau} \mathrm{e}^{-\left(l_{\mathrm{V}}+\hat{s}_{\beta}\right)(T-s)} E_{1}(s, \omega) \mathrm{d} s \\
& +n_{\mathrm{V}} \hat{r}_{\beta} \int_{0}^{\tau} \mathrm{e}^{-\left(l_{\mathrm{v}}+\hat{p}_{\beta}\right)(T-s)} E_{1}(s, \omega) \mathrm{d} s+n_{\mathrm{V}} \hat{q}_{\beta} \int_{0}^{\tau} \mathrm{e}^{-l_{\mathrm{V}}(T-s)} E_{1}(s, \omega) \mathrm{d} s .
\end{aligned}
$$


Besides it, it can be shown as well that

$$
\begin{aligned}
& \int_{0}^{\tau} \mathbb{E}\left[\beta(T-s)^{2}\right] E_{1}^{2}(s, \omega) \mathrm{d} s \\
& =\int_{0}^{\tau} \mathrm{e}^{-s_{\beta}(T-s)} E_{1}^{2}(s, \omega) \mathrm{d} s+r_{\beta} \int_{0}^{\tau} \mathrm{e}^{-p_{\beta}(T-s)} E_{1}^{2}(s, \omega) \mathrm{d} s+q_{\mathrm{b}} \int_{0}^{\tau} E_{1}^{2}(s, \omega) \mathrm{d} s,
\end{aligned}
$$

and

$$
\begin{aligned}
& \int_{0}^{\tau} \mathbb{E}\left[\eta(T-s)^{2}\right] F_{1}^{2}(s, \omega) \mathrm{d} s \\
& =\int_{0}^{\tau} \mathrm{e}^{-s_{\eta}(T-s)} F_{1}^{2}(s, \omega) \mathrm{d} s+r_{\eta} \int_{0}^{\tau} \mathrm{e}^{-p_{\eta}(T-s)} F_{1}^{2}(s, \omega) \mathrm{d} s+q_{\eta} \int_{0}^{\tau} F_{1}^{2}(s, \omega) \mathrm{d} s .
\end{aligned}
$$

Hence, the only integrals to be computed are of the following form:

$$
\int_{0}^{\tau} \mathrm{e}^{-a(T-s)} E_{1}^{2}(s, \omega) \mathrm{d} s \text { and } \int_{0}^{\tau} \mathrm{e}^{-a(T-s)} F_{1}^{2}(s, \omega) \mathrm{d} s
$$

since all the other integrals

$$
\begin{aligned}
& \int_{0}^{\tau} E_{1}(s, \omega) \mathrm{d} s, \int_{0}^{\tau} F_{1}(s, \omega) \mathrm{d} s, \int_{0}^{\tau} \mathrm{e}^{-a(T-s)} E_{1}(s, \omega) \mathrm{d} s, \\
& \int_{0}^{\tau} \mathrm{e}^{-a(T-s)} F_{1}(s, \omega) \mathrm{d} s, \int_{0}^{\tau} E_{1}^{2}(s, \omega) \mathrm{d} s, \int_{0}^{\tau} F_{1}^{2}(s, \omega) \mathrm{d} s
\end{aligned}
$$

have been already computed in Appendix A.2.

In particular, it can be proved that

$$
\begin{aligned}
& \int_{0}^{\tau} \mathrm{e}^{-a(T-s)} F_{1}^{2}(s, \omega) \mathrm{d} s \\
& =\frac{\left(\mu_{\mathrm{V}}-v(0)\right)^{2}}{\left(\kappa_{\eta}+\kappa_{\mathrm{V}}-\bar{B}_{3}\right)^{2}} \hat{B}_{1}^{2} \mathrm{e}^{-\left(a+2 \kappa_{\mathrm{V}}\right) T} \frac{\mathrm{e}^{\left(a+2\left(\kappa_{\mathrm{V}}-\bar{B}_{3}\right)\right) \tau}-1}{a+2\left(\kappa_{\mathrm{V}}-\bar{B}_{3}\right)} \\
& +\frac{\left(v(0)-\mu_{\mathrm{V}}\right)^{2}}{\left(\kappa_{\eta}+\kappa_{\mathrm{V}}\right)^{2}} \hat{B}_{1}^{2} \mathrm{e}^{-\left(a+2 \kappa_{\mathrm{V}}\right) T} \frac{\mathrm{e}^{\left(a+2 \kappa_{\mathrm{V}}\right) \tau}-1}{a+2 \kappa_{\mathrm{V}}}+\frac{\mu_{\mathrm{V}}^{2}}{\kappa_{\eta}^{2}} \hat{B}_{1}^{2} \mathrm{e}^{-a T} \frac{\mathrm{e}^{a \tau}-1}{a} \\
& +\frac{\mu_{\mathrm{V}}^{2}}{\left(\kappa_{\eta}-\bar{B}_{3}\right)^{2}} \hat{B}_{1}^{2} \mathrm{e}^{-a T} \frac{\mathrm{e}^{\left(a-2 \bar{B}_{3}\right) \tau}-1}{a-2 \bar{B}_{3}}+\frac{\hat{B}_{1}^{2} \hat{B}_{2}^{2} \mathrm{e}^{-a T}\left(\mathrm{e}^{\left(a-\kappa_{\eta}\right) \tau}-1\right)}{a-\kappa_{\eta}} \\
& +2 \frac{\left(\mu_{\mathrm{V}}-v(0)\right)\left(v(0)-\mu_{\mathrm{V}}\right)}{\left(\kappa_{\eta}+\kappa_{\mathrm{V}}\right)\left(\kappa_{\eta}+\kappa_{\mathrm{V}}-\bar{B}_{3}\right)} \hat{B}_{1}^{2} \mathrm{e}^{-\left(a+2 \kappa_{\mathrm{V}}\right) T} \frac{\mathrm{e}^{\left(a+2 \kappa_{\mathrm{V}}-\bar{B}_{3}\right) \tau}-1}{a+2 \kappa_{\mathrm{V}}-\bar{B}_{3}} \\
& +2 \frac{\mu_{\mathrm{V}}-v(0) \mu_{\mathrm{V}}}{\kappa_{\eta}\left(\kappa_{\eta}+\kappa_{\mathrm{V}}-\bar{B}_{3}\right)} \hat{B}_{1}^{2} \mathrm{e}^{-\left(a+\kappa_{\mathrm{V}}\right) T} \frac{\mathrm{e}^{\left(a+\kappa_{\mathrm{V}}-\bar{B}_{3}\right) \tau}-1}{a+\kappa_{\mathrm{V}}-\bar{B}_{3}} \\
& -2 \frac{\mu_{\mathrm{V}}\left(\mu_{\mathrm{V}}-v(0)\right)}{\left(\kappa_{\eta}-\bar{B}_{3}\right)\left(\kappa_{\eta}+\kappa_{\mathrm{V}}-\bar{B}_{3}\right)} \hat{B}_{1}^{2} \mathrm{e}^{-\left(\kappa_{\mathrm{V}}+a\right) T} \frac{\mathrm{e}^{\left(a+\kappa_{\mathrm{V}}-2 \bar{B}_{3}\right) \tau}-1}{a+\kappa_{\mathrm{V}}-2 \bar{B}_{3}} \\
& +2 \frac{\mu_{\mathrm{V}}-v(0)}{\kappa_{\eta}+\kappa_{\mathrm{V}}-\bar{B}_{3}} \hat{B}_{1}^{2} \hat{B}_{2} \mathrm{e}^{-\left(a+\kappa_{\mathrm{V}}\right) T} \frac{\mathrm{e}^{\left(a+\kappa_{\mathrm{V}}-\bar{B}_{3}-\kappa_{\eta}\right) \tau}-1}{a+\kappa_{\mathrm{V}}-\bar{B}_{3}-\kappa_{\eta}} \\
& +2 \frac{\left(v(0)-\mu_{\mathrm{V}}\right) \mu_{\mathrm{V}}}{\kappa_{\eta}\left(\kappa_{\mathrm{V}}+\kappa_{\eta}\right)} \hat{B}_{1}^{2} \mathrm{e}^{-\left(a+\kappa_{\mathrm{V}}\right) T} \frac{\mathrm{e}^{a \tau}-1}{a}-2 \frac{\mu_{\mathrm{V}}\left(v(0)-\mu_{\mathrm{V}}\right)}{\left(\kappa_{\eta}+\kappa_{\mathrm{V}}\right)\left(\kappa_{\eta}-\bar{B}_{3}\right)} \hat{B}_{1}^{2} \\
& \times \mathrm{e}^{-\left(a+\kappa_{\mathrm{V}}\right) T} \frac{\mathrm{e}^{\left(a+\kappa_{\mathrm{V}}-\kappa_{\eta}\right) \tau}-1}{a+\kappa_{\mathrm{V}}-\kappa_{\eta}}+2 \frac{v(0)-\mu_{\mathrm{V}}}{\kappa_{\eta}+\kappa_{\mathrm{V}}} \hat{B}_{1}^{2} \hat{B}_{2} \mathrm{e}^{-\left(a+\kappa_{\mathrm{V}}\right) T}
\end{aligned}
$$




$$
\begin{aligned}
& \times \frac{\mathrm{e}^{\left(a+\kappa_{\mathrm{V}}-\kappa_{\eta}\right) \tau}-1}{a+\kappa_{\mathrm{V}}-\kappa_{\eta}}-2 \frac{\mu_{\mathrm{V}}^{2}}{\kappa_{\eta}\left(\kappa_{\eta}-\bar{B}_{3}\right)} \hat{B}_{1}^{2} \mathrm{e}^{-a T} \frac{\mathrm{e}^{\left(a-\bar{B}_{3}\right) \tau}-1}{a-\bar{B}_{3}} \\
& +2 \frac{\mu_{\mathrm{V}}}{\kappa_{\eta}} \hat{B}_{1}^{2} \hat{B}_{2} \mathrm{e}^{-a T} \frac{\mathrm{e}^{\left(a-\kappa_{\eta}\right) \tau}-1}{a-\kappa_{\eta}}-2 \frac{\mu_{\mathrm{V}}}{\kappa_{\eta}-\bar{B}_{3}} \hat{B}_{1}^{2} \hat{B}_{2} \mathrm{e}^{-a T} \frac{\mathrm{e}^{\left(a-\bar{B}_{3}-\kappa_{\eta}\right) \tau}-1}{a-\bar{B}_{3}-\kappa_{\eta}} .
\end{aligned}
$$

and that

$$
\begin{aligned}
& \int_{0}^{\tau} \mathrm{e}^{-a(T-s)} E_{1}^{2}(s, \omega) \mathrm{d} s \\
& =-\omega^{2}\left[\frac{m_{\mathrm{V}}^{2} m_{\mathrm{U}}^{2}}{\kappa_{\beta}^{2}} \mathrm{e}^{-a T}\left(\frac{\mathrm{e}^{a \tau}-1}{a}+\frac{\mathrm{e}^{\left(a-2 \kappa_{\beta}\right) \tau}-1}{a-2 \kappa_{\beta}}-2 \frac{\mathrm{e}^{\left(a-\kappa_{\beta}\right) \tau}-1}{a-\kappa_{\beta}}\right)\right. \\
& +\frac{m_{\mathrm{V}}^{2} n_{\mathrm{U}}^{2}}{\left(\kappa_{\beta}+l_{\mathrm{U}}\right)^{2}} \mathrm{e}^{-\left(a+2 l_{\mathrm{U}}\right) T}\left(\frac{\mathrm{e}^{\left(a+2 l_{\mathrm{U}}\right) \tau}-1}{a+2 l_{\mathrm{U}}}+\frac{\mathrm{e}^{\left(a-2 \kappa_{\beta}\right) \tau}-1}{a-2 \kappa_{\beta}}-2 \frac{\mathrm{e}^{\left(a+l_{\mathrm{U}}-\kappa_{\beta}\right) \tau}-1}{a+l_{\mathrm{U}}-\kappa_{\beta}}\right) \\
& +\frac{m_{\mathrm{U}}^{2} n_{\mathrm{V}}^{2}}{\left(\kappa_{\beta}+l_{\mathrm{V}}\right)^{2}} \mathrm{e}^{-\left(a+2 l_{\mathrm{V}}\right) T}\left(\frac{\mathrm{e}^{\left(a+2 l_{\mathrm{V}}\right) \tau}-1}{a+2 l_{\mathrm{V}}}+\frac{\mathrm{e}^{\left(a-2 \kappa_{\beta}\right) \tau}-1}{a-2 \kappa_{\beta}}-2 \frac{\mathrm{e}^{\left(a+l_{\mathrm{V}}-\kappa_{\beta}\right) \tau}-1}{a+l_{\mathrm{V}}-\kappa_{\beta}}\right) \\
& +\frac{n_{\mathrm{V}}^{2} n_{\mathrm{U}}^{2}}{\left(\kappa_{\beta}+l_{\mathrm{U}}+l_{\mathrm{V}}\right)^{2}} \mathrm{e}^{-\left(a+2\left(l_{\mathrm{U}}+l_{\mathrm{V}}\right)\right) T}\left(\frac{\mathrm{e}^{\left(a+2\left(l_{\mathrm{U}}+l_{\mathrm{V}}\right)\right) \tau}-1}{a+2\left(l_{\mathrm{U}}+l_{\mathrm{V}}\right)}+\frac{\mathrm{e}^{\left(a-2 \kappa_{\beta}\right) \tau}-1}{a-2 \kappa_{\beta}}\right. \\
& \left.-2 \frac{\mathrm{e}^{\left(a+l_{\mathrm{U}}+l_{\mathrm{V}}-\kappa_{\beta}\right) \tau}-1}{a+l_{\mathrm{U}}+l_{\mathrm{V}}-\kappa_{\beta}}\right)+2 \frac{m_{\mathrm{V}}^{2} m_{\mathrm{U}} n_{\mathrm{U}}}{\kappa_{\beta}\left(\kappa_{\beta}+l_{\mathrm{U}}\right)} \mathrm{e}^{-\left(a+l_{\mathrm{U}}\right) T}\left(\frac{\mathrm{e}^{\left(a+l_{\mathrm{U}}\right) \tau}-1}{a+l_{\mathrm{U}}}\right. \\
& \left.-\frac{\mathrm{e}^{\left(a-\kappa_{\beta}\right) \tau}-1}{a-\kappa_{\beta}}-\frac{\mathrm{e}^{\left(a+l_{\mathrm{U}}-\kappa_{\beta}\right) \tau}-1}{a+l_{\mathrm{U}}-\kappa_{\beta}}+\frac{\mathrm{e}^{\left(a-2 \kappa_{\beta}\right) \tau}-1}{a-2 \kappa_{\beta}}\right)+2 \frac{m_{\mathrm{V}} m_{\mathrm{U}}^{2} n_{\mathrm{V}}}{\kappa_{\beta}\left(\kappa_{\beta}+l_{\mathrm{V}}\right)} \\
& \times \mathrm{e}^{-\left(a+l_{\mathrm{V}}\right) T}\left(\frac{\mathrm{e}^{\left(a+l_{\mathrm{V}}\right) \tau}-1}{a+l_{\mathrm{V}}}-\frac{\mathrm{e}^{\left(a-\kappa_{\beta}\right) \tau}-1}{a-\kappa_{\beta}}-\frac{\mathrm{e}^{\left(a+l_{\mathrm{V}}-\kappa_{\beta}\right) \tau}-1}{a+l_{\mathrm{V}}-\kappa_{\beta}}\right. \\
& \left.+\frac{\mathrm{e}^{\left(a-2 \kappa_{\beta}\right) \tau}-1}{a-2 \kappa_{\beta}}\right)+2 \frac{m_{\mathrm{U}} m_{\mathrm{V}} n_{\mathrm{V}} n_{\mathrm{U}}}{\kappa_{\beta}\left(\kappa_{\beta}+l_{\mathrm{U}}+l_{\mathrm{V}}\right)} \mathrm{e}^{-\left(a+l_{\mathrm{U}}+l_{\mathrm{V}}\right) T}\left(\frac{\mathrm{e}^{\left(a+l_{\mathrm{U}}+l_{\mathrm{V}}\right) \tau}-1}{a+l_{\mathrm{U}}+l_{\mathrm{V}}}\right. \\
& \left.-\frac{\mathrm{e}^{\left(a-\kappa_{\beta}\right) \tau}-1}{a-\kappa_{\beta}}-\frac{\mathrm{e}^{\left(a+l_{\mathrm{U}}+l_{\mathrm{V}}-\kappa_{\beta}\right) \tau}-1}{a+l_{\mathrm{U}}+l_{\mathrm{V}}-\kappa_{\beta}}+\frac{\mathrm{e}^{\left(a-2 \kappa_{\beta}\right) \tau}-1}{a-2 \kappa_{\beta}}\right) \\
& +2 \frac{m_{\mathrm{V}} m_{\mathrm{U}} n_{\mathrm{V}} n_{\mathrm{U}}}{\left(\kappa_{\beta}+l_{\mathrm{U}}\right)\left(\kappa_{\beta}+l_{\mathrm{V}}\right)} \mathrm{e}^{-\left(a+l_{\mathrm{U}}+l_{\mathrm{V}}\right) T}\left(\frac{\mathrm{e}^{\left(a+l_{\mathrm{U}}+l_{\mathrm{V}}\right) \tau}-1}{a+l_{\mathrm{U}}+l_{\mathrm{V}}}\right. \\
& \left.-\frac{\mathrm{e}^{\left(a+l_{\mathrm{U}}-\kappa_{\beta}\right) \tau}-1}{a+l_{\mathrm{U}}-\kappa_{\beta}}-\frac{\mathrm{e}^{\left(a+l_{\mathrm{V}}-\kappa_{\beta}\right) \tau}-1}{a+l_{\mathrm{V}}-\kappa_{\beta}}+\frac{\mathrm{e}^{\left(a-2 \kappa_{\beta}\right) \tau}-1}{a-2 \kappa_{\beta}}\right) \\
& +2 \frac{m_{\mathrm{V}} n_{\mathrm{U}}^{2} n_{\mathrm{V}}}{\left(\kappa_{\beta}+l_{\mathrm{U}}\right)\left(\kappa_{\beta}+l_{\mathrm{U}}+l_{\mathrm{V}}\right)} \mathrm{e}^{-\left(a+2 l_{\mathrm{U}}+l_{\mathrm{V}}\right) T}\left(\frac{\mathrm{e}^{\left(a+2 l_{\mathrm{U}}+l_{\mathrm{V}}\right) \tau}-1}{a+2 l_{\mathrm{U}}+l_{\mathrm{V}}}\right. \\
& \left.-\frac{\mathrm{e}^{\left(a+l_{\mathrm{U}}-\kappa_{\beta}\right) \tau}-1}{a+l_{\mathrm{U}}-\kappa_{\beta}}-\frac{\mathrm{e}^{\left(a+l_{\mathrm{U}}+l_{\mathrm{V}}-\kappa_{\beta}\right) \tau}-1}{a+l_{\mathrm{U}}+l_{\mathrm{V}}-\kappa_{\beta}}+\frac{\mathrm{e}^{\left(a-2 \kappa_{\beta}\right) \tau}-1}{a-2 \kappa_{\beta}}\right)
\end{aligned}
$$




$$
\begin{aligned}
& +2 \frac{m_{\mathrm{U}} n_{\mathrm{V}}^{2} n_{\mathrm{U}}}{\left(\kappa_{\beta}+l_{\mathrm{V}}\right)\left(\kappa_{\beta}+l_{\mathrm{U}}+l_{\mathrm{V}}\right)} \mathrm{e}^{-\left(a+2 l_{\mathrm{V}}+l_{\mathrm{U}}\right) T}\left(\frac{\mathrm{e}^{\left(a+2 l_{\mathrm{V}}+l_{\mathrm{U}}\right) \tau}-1}{a+2 l_{\mathrm{V}}+l_{\mathrm{U}}}\right. \\
& \left.\left.-\frac{\mathrm{e}^{\left(a+l_{\mathrm{V}}-\kappa_{\beta}\right) \tau}-1}{a+l_{\mathrm{V}}-\kappa_{\beta}}-\frac{\mathrm{e}^{\left(a+l_{\mathrm{U}}+l_{\mathrm{V}}-\kappa_{\beta}\right) \tau}-1}{a+l_{\mathrm{U}}+l_{\mathrm{V}}-\kappa_{\beta}}+\frac{\mathrm{e}^{\left(a-2 \kappa_{\beta}\right) \tau}-1}{a-2 \kappa_{\beta}}\right)\right],
\end{aligned}
$$

Collecting all the terms together completes the proof. 